\title{
Rh-Catalyzed [2+2+2] Cycloadditions with Benzoquinones: De Novo Access to Naphthoquinones for Lignan and Type II Polyketide Synthesis
}

\author{
James M. Wood, ${ }^{\dagger}$ Eufrânio N. da Silva Júnior, ${ }^{\dagger *}$ and John F. Bower ${ }^{\dagger *}$ \\ $†$ School of Chemistry, University of Bristol, Bristol, BS8 1TS, United Kingdom \\ $\ddagger$ Institute of Exact Sciences, Department of Chemistry, Federal University of Minas Gerais, CEP \\ 31270-901, Belo Horizonte, MG, Brazil
}

\section{Supporting Information}

\section{Table of Contents}

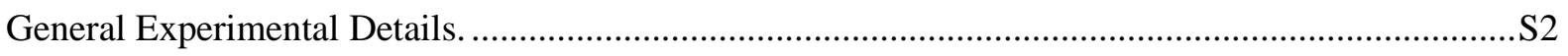

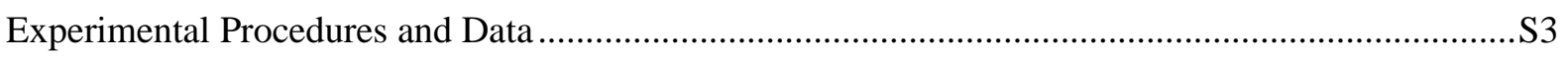

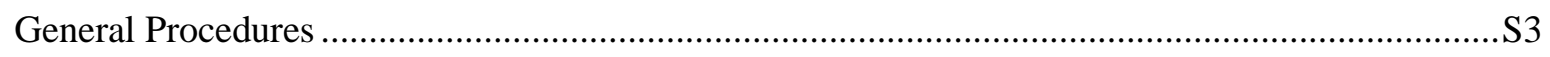

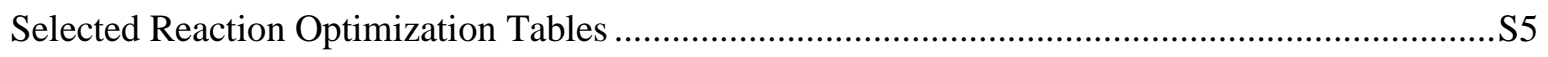

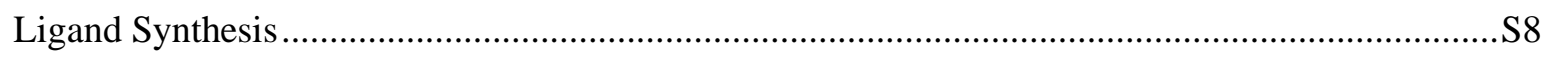

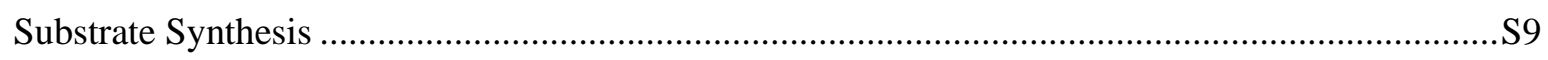

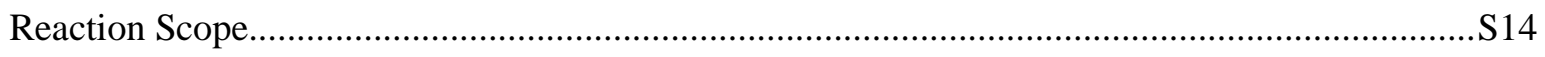

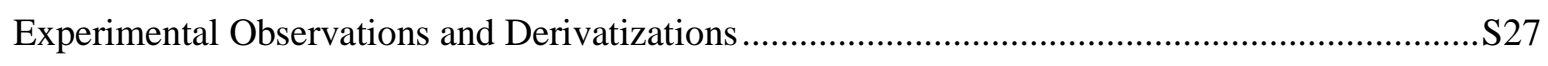

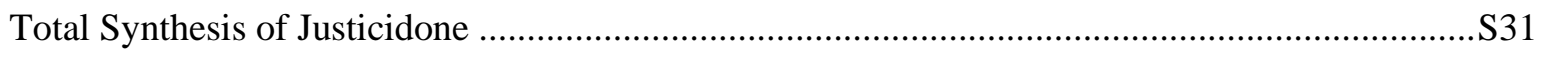

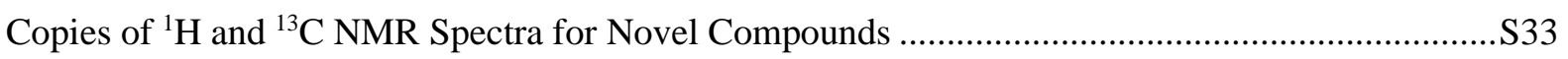

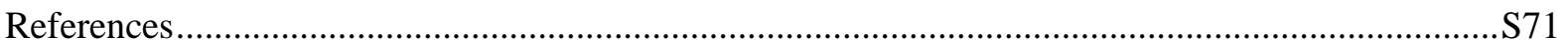

The experiments specifically identified in the main text as being in the supporting information are highlighted in blue. 
General Experimental Details. Starting materials sourced from commercial suppliers (Acros, Aldrich, Alfa Aesar, Fluorochem, TCI) were used as received unless otherwise stated. Anhydrous 1,2dichloroethane was sourced from Aldrich and used as received. Other dry solvents, where necessary, were obtained by distillation using standard procedures or by passage through a column of anhydrous alumina using equipment from Anhydrous Engineering. Petrol refers to the fraction of petroleum ether boiling in the range of $40-60{ }^{\circ} \mathrm{C}$. The removal of solvents in vacuo was achieved using both a Büchi rotary evaporator (bath temperatures up to $45^{\circ} \mathrm{C}$ ) at a pressure of either 4 mbar (diaphragm pump) or 1 mbar (oil pump), as appropriate, and a high vacuum line at room temperature. Reactions requiring anhydrous conditions were run under an atmosphere of dry nitrogen or argon; glassware was either flame dried immediately prior to use or placed in an oven $\left(200{ }^{\circ} \mathrm{C}\right)$ for at least $2 \mathrm{~h}$ and allowed to cool either in a desiccator or under an atmosphere of nitrogen or argon; liquid reagents, solutions or solvents were added via syringe through rubber septa; solid reagents were added via Schlenk type adapters. Commercially available Merck Kieselgel 60F254 aluminium backed plates were used for TLC analysis. Visualisation was achieved by either UV fluorescence, basic $\mathrm{KMnO}_{4}$ solution and heat. Flash column chromatography was performed using silica gel (Aldrich 40-63 $\mu \mathrm{m}, 230-400$ mesh). The crude material was applied to the column as a solution in $\mathrm{CH}_{2} \mathrm{Cl}_{2}$ or by pre-adsorption onto silica, as appropriate. Melting points were determined using a Reichert melting point table and temperature controller and are uncorrected. Infrared spectra were recorded in the range 4000-600 $\mathrm{cm}^{-1}$ on a Perkin Elmer Spectrum either as neat films or solids compressed onto a diamond window. Abbreviations used are: w (weak), m (medium) or s (strong). NMR spectra were recorded using either a Varian 400-MR, or Bruker Nano 400. Chemical shifts $(\delta)$ are quoted in parts per million ( $\mathrm{ppm})$, coupling constants $(\mathrm{J})$ are given in $\mathrm{Hz}$ to the nearest $0.5 \mathrm{~Hz}$. Other abbreviations used are s (singlet), d (doublet), t (triplet), q (quartet), p (pentet), $\mathrm{m}$ (multiplet) and br (broad). ${ }^{1} \mathrm{H}$ and ${ }^{13} \mathrm{C}$ NMR spectra were referenced to the appropriate residual solvent peak. ${ }^{19} \mathrm{~F}$ spectra were referenced to $\mathrm{CCl}_{3} \mathrm{~F}$ as an external standard, ${ }^{31} \mathrm{P}$ spectra were referenced to $\mathrm{H}_{3} \mathrm{PO}_{4}$ as an external standard. Assignments of ${ }^{1} \mathrm{H}$ NMR and ${ }^{13} \mathrm{C}$ NMR signals were made, where possible, using COSY, HSQC and HMBC. Mass spectra were determined by the University of Bristol mass spectrometry service using a Shimadzu GCMS QP2010+ (EI+ mode), Brüker Daltonics FT-ICRMS Apex 4e 7.0T FT-MS (ESI+ mode), Thermo Scientific Orbitrap Elite (APCI mode). 


\section{Experimental Procedures and Data}

\section{General Procedures}

\section{General Procedure A: Reaction condition screening for the $(2+2+2)$ cycloaddition of 1a with 2a}

A flame-dried Schlenk tube, fitted with a magnetic stirrer, was charged with diyne 1a, quinone 2a, catalyst and ligand. The Schlenk tube was fitted with a rubber septum, evacuated and refilled with nitrogen (three cycles), then solvent was added via syringe. The tube was sealed with a PTFE cap and placed in a pre-heated heating block at the specified temperature for $18 \mathrm{~h}$ unless otherwise noted. The reaction mixture was cooled to room temperature and concentrated in vacuo. Yields were determined by ${ }^{1} \mathrm{H}$ NMR analysis of the crude reaction mixture using 1,4-dinitrobenzene as an internal standard.

\section{General Procedure B: $(2+2+2)$ Cycloadditions of diynes 1 with quinones 2}

A flame-dried reaction tube, fitted with a magnetic stirrer, was charged with $\left[\mathrm{Rh}(\mathrm{coe})_{2} \mathrm{Cl}\right]_{2}(3.75 \mathrm{~mol} \%)$, $\left(4-\mathrm{NCC}_{6} \mathrm{H}_{4}\right)_{3} \mathrm{P}(15 \mathrm{~mol} \%)$ and the appropriate quinone substrate $2(200 \mathrm{~mol} \%)$. The tube was flushed with argon, then fitted with a rubber septum, placed under a balloon of argon, and DCE (0.4 M) was added via syringe. The reaction tube was placed in a preheated heating block at $70{ }^{\circ} \mathrm{C}$, then the appropriate diyne substrate $\mathbf{1}(100 \mathrm{~mol} \%)$ in DCE $(0.13 \mathrm{M})$ was added dropwise by syringe pump over $2 \mathrm{~h}$. After addition of diyne 1 was complete, the reaction mixture was stirred at $70{ }^{\circ} \mathrm{C}$ for $16 \mathrm{~h}$, then cooled to room temperature and concentrated in vacuo. The crude reaction mixture was purified by flash column chromatography to yield the target naphthoquinone $\mathbf{3}$.

Note: the reaction generates a stoichiometric amount of a hydroquinone by-product from the quinone 2, which can be difficult to separate from the product 3 by FCC. In such cases, the crude reaction mixture can be dissolved in EtOAc and washed twice with $0.5 \mathrm{M}$ aqueous $\mathrm{KOH}$ to remove this hydroquinone by-product prior to FCC.

\section{General Procedure C: $(2+2+2)$ Cycloadditions of diynes 1 with quinones 2}

A flame-dried Schlenk tube, fitted with a magnetic stirrer, was charged with $\left[\mathrm{Rh}(\mathrm{coe}){ }_{2} \mathrm{Cl}\right]_{2}(3.75 \mathrm{~mol} \%)$, (4- $\left.\mathrm{NCC}_{6} \mathrm{H}_{4}\right)_{3} \mathrm{P}(15 \mathrm{~mol} \%)$ and the appropriate quinone substrate $2(200 \mathrm{~mol} \%$ or $500 \mathrm{~mol} \%$, as specified). The Schlenk tube was fitted with a rubber septum, evacuated and refilled with nitrogen (three cycles), then DCE ( $0.4 \mathrm{M})$ was added via syringe. The reaction tube was placed in a preheated heating block at $70{ }^{\circ} \mathrm{C}$, then the appropriate diyne substrate 1 (100 mol\%) in DCE $(0.13 \mathrm{M})$ was added dropwise by syringe pump over $2 \mathrm{~h}$. After addition of diyne 1 was complete, the Schlenk tube was sealed with a PTFE cap and heated at $100{ }^{\circ} \mathrm{C}$ in a pre-heated heating block for $16 \mathrm{~h}$, then cooled to room temperature 
and concentrated in vacuo. The crude reaction mixture was purified by flash column chromatography to yield the target naphthoquinone $\mathbf{3}$. 


\section{Selected Reaction Optimization Tables}

\section{Rhodium Catalyst Screening Table}

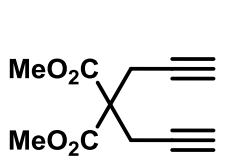

1 a<smiles>O=C1C=CC(=O)C=C1</smiles>

$2 a$ $300 \mathrm{~mol} \%$

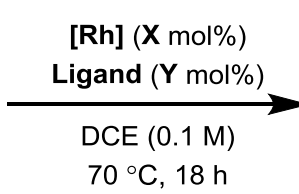

General Procedure A
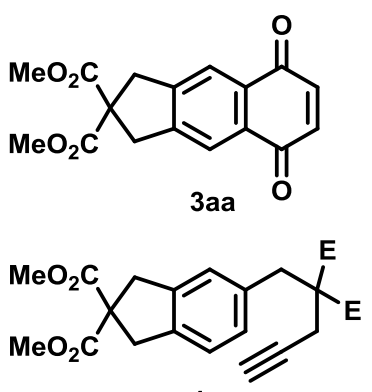

\begin{tabular}{|c|c|c|c|}
\hline Entry & Pre-catalyst (X) & Ligand $(Y)$ & 1a:3aa:4 ${ }^{[a]}$ \\
\hline 1 & {$[\mathrm{Rh}(\mathrm{cod}) \mathrm{Cl}]_{2}(5)$} & BINAP (10) & $0: 39: 8$ \\
\hline 2 & {$[\mathrm{Rh}(\mathrm{cod}) \mathrm{OMe}]_{2}(5)$} & BINAP (10) & $0: 27: 8$ \\
\hline 3 & {$[\mathrm{Rh}(\mathrm{cod}) \mathrm{OH}]_{2}(5)$} & BINAP (10) & $0: 18: 11$ \\
\hline 4 & {$\left[\mathrm{Rh}(\mathrm{cod})_{2}\right] \mathrm{OTf}(10)$} & BINAP (10) & $0: 32: 3$ \\
\hline 5 & {$\left[\mathrm{Rh}(\operatorname{cod})_{2}\right] \mathrm{BF}_{4}(10)$} & BINAP (10) & $0: 12: 4$ \\
\hline 6 & {$\left[\mathrm{Rh}(\mathrm{cod})_{2}\right] \mathrm{SbF}_{6}(10)$} & BINAP (10) & $0: 9: 6$ \\
\hline 7 & {$\left[\mathrm{Rh}(\operatorname{cod})_{2}\right]$ BARF $(10)$} & BINAP (10) & $0: 11: 2$ \\
\hline 8 & {$[\mathrm{Rh}(\operatorname{cod}) \mathrm{Cl}]_{2}(5)$} & $\left(4-\mathrm{NCC}_{6} \mathrm{H}_{4}\right)_{3} \mathrm{P}(20)$ & $0: 49: 14$ \\
\hline 9 & $\left.[\mathrm{Rh}(\mathrm{coe}))_{2} \mathrm{Cl}\right]_{2}(5)$ & $\left(4-\mathrm{NCC}_{6} \mathrm{H}_{4}\right)_{3} \mathrm{P}(20)$ & $0: 58: 2$ \\
\hline 10 & {$[\mathrm{Rh}(\mathrm{nbd}) \mathrm{Cl}]_{2}(5)$} & $\left(4-\mathrm{NCC}_{6} \mathrm{H}_{4}\right)_{3} \mathrm{P}(20)$ & $0: 50: 17$ \\
\hline 11 & {$\left[\mathrm{Rh}(\mathrm{CO})_{2} \mathrm{Cl}\right]_{2}(5)$} & $\left(4-\mathrm{NCC}_{6} \mathrm{H}_{4}\right)_{3} \mathrm{P}(20)$ & $33: 9: 11$ \\
\hline
\end{tabular}

[a] Yields were determined by $1 \mathrm{H}$ NMR analysis using 1,4-dinitrobenzene as an internal standard 


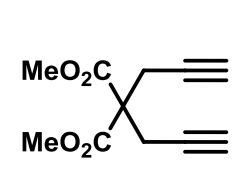

$1 a$<smiles>O=C1C=CC(=O)C=C1</smiles>

2a

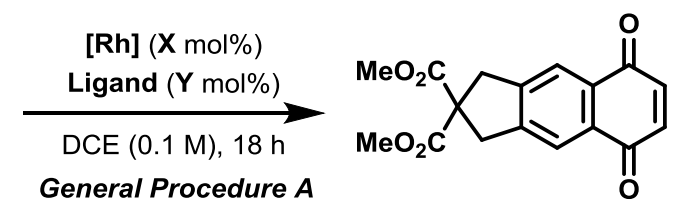

3aa

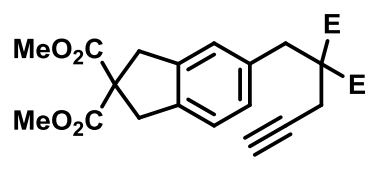

4

$300 \mathrm{~mol} \%$

\begin{tabular}{|c|c|c|c|c|}
\hline Entry & Pre-catalyst (X) & Ligand (Y) & $\mathrm{T}\left({ }^{\circ} \mathrm{C}\right)$ & $1 \mathrm{a}: 3 \mathrm{aa}: 4^{[\mathrm{a}]}$ \\
\hline 1 & {$[\mathrm{Rh}(\operatorname{cod}) \mathrm{Cl}]_{2}(5)$} & none & 70 & $0: 21: 2$ \\
\hline 2 & {$\left[\mathrm{Rh}(\mathrm{coe})_{2} \mathrm{Cl}\right]_{2}(5)$} & none & 50 & $2: 36: 8$ \\
\hline 3 & {$[\mathrm{Rh}(\operatorname{cod}) \mathrm{Cl}]_{2}(5)$} & BINAP (10) & 70 & $0: 39: 8$ \\
\hline 4 & {$[\mathrm{Rh}(\operatorname{cod}) \mathrm{Cl}]_{2}(5)$} & $\mathrm{H}_{8}$-BINAP (10) & 70 & $0: 36: 8$ \\
\hline 5 & {$\left[\mathrm{Rh}(\operatorname{cod}) \mathrm{Cl}_{2}(5)\right.$} & Tol-BINAP (10) & 70 & $0: 41: 0$ \\
\hline 6 & {$[\mathrm{Rh}(\operatorname{cod}) \mathrm{Cl}]_{2}(5)$} & SEGPhos (10) & 70 & $0: 27: 8$ \\
\hline 7 & {$[\mathrm{Rh}(\operatorname{cod}) \mathrm{Cl}]_{2}(5)$} & BIPHEP (10) & 70 & $0: 26: 7$ \\
\hline 8 & {$[\mathrm{Rh}(\operatorname{cod}) \mathrm{Cl}]_{2}(5)$} & dppe (10) & 70 & $16: 3: 7$ \\
\hline 9 & {$[\mathrm{Rh}(\operatorname{cod}) \mathrm{Cl}]_{2}(5)$} & dfppe (10) & 70 & $0: 16: 25$ \\
\hline 10 & {$[\mathrm{Rh}(\operatorname{cod}) \mathrm{Cl}]_{2}(5)$} & dppp (10) & 70 & $24: 5: 5$ \\
\hline 11 & {$[\mathrm{Rh}(\operatorname{cod}) \mathrm{Cl}]_{2}(5)$} & dppf (10) & 70 & $0: 17: 7$ \\
\hline 12 & {$[\mathrm{Rh}(\operatorname{cod}) \mathrm{Cl}]_{2}(5)$} & XantPhos (10) & 70 & $7: 11: 18$ \\
\hline 13 & {$[\mathrm{Rh}(\operatorname{cod}) \mathrm{Cl}]_{2}(5)$} & $\mathrm{PPh}_{3}(20)$ & 70 & $11: 5: 5$ \\
\hline 14 & {$[\mathrm{Rh}(\operatorname{cod}) \mathrm{Cl}]_{2}(5)$} & $\mathrm{Cy}_{3} \mathrm{P}(20)$ & 70 & $20: 1: 2$ \\
\hline 15 & {$[\mathrm{Rh}(\operatorname{cod}) \mathrm{Cl}]_{2}(5)$} & $(\text { o-anisyl })_{3} \mathrm{P}(20)$ & 70 & $36: 4: 0$ \\
\hline 16 & {$[\mathrm{Rh}(\operatorname{cod}) \mathrm{Cl}]_{2}(5)$} & $(o-T o l){ }_{3} P(20)$ & 70 & $16: 5: 8$ \\
\hline 17 & {$[\mathrm{Rh}(\operatorname{cod}) \mathrm{Cl}]_{2}(5)$} & (furyl) ${ }_{3} \mathrm{P}(20)$ & 70 & $0: 23: 30$ \\
\hline 18 & {$[\mathrm{Rh}(\operatorname{cod}) \mathrm{Cl}]_{2}(5)$} & $\left(4-\mathrm{FC}_{6} \mathrm{H}_{4}\right)_{3} \mathrm{P}(20)$ & 70 & $18: 6: 15$ \\
\hline 19 & {$[\mathrm{Rh}(\operatorname{cod}) \mathrm{Cl}]_{2}(5)$} & $\left(4-\mathrm{NCC}_{6} \mathrm{H}_{4}\right)_{3} \mathrm{P}(20)$ & 70 & $0: 47: 14$ \\
\hline 20 & {$[\mathrm{Rh}(\operatorname{cod}) \mathrm{Cl}]_{2}(5)$} & $\left(4-\left(\mathrm{CF}_{3}\right) \mathrm{C}_{6} \mathrm{H}_{4}\right)_{3} \mathrm{P}(20)$ & 70 & $0: 13: 18$ \\
\hline 21 & {$[\mathrm{Rh}(\operatorname{cod}) \mathrm{Cl}]_{2}(5)$} & $\left(3,4,5-\mathrm{F}_{3} \mathrm{C}_{6} \mathrm{H}_{2}\right)_{3} \mathrm{P}(20)$ & 70 & $0: 22: 26$ \\
\hline 22 & {$[\mathrm{Rh}(\operatorname{cod}) \mathrm{Cl}]_{2}(5)$} & $\left(3,5-\left(\mathrm{CF}_{3}\right)_{2} \mathrm{C}_{6} \mathrm{H}_{3}\right)_{3} \mathrm{P}(20)$ & 70 & $0: 22: 27$ \\
\hline 23 & {$[\mathrm{Rh}(\operatorname{cod}) \mathrm{Cl}]_{2}(5)$} & ${ }^{\mathrm{Me}} \mathrm{CgPPh}(20)$ & 70 & $0: 27: 0$ \\
\hline 24 & {$\left[\mathrm{Rh}(\mathrm{coe})_{2} \mathrm{Cl}\right]_{2}(5)$} & ${ }^{\mathrm{Me}} \mathrm{CgP}\left(4-\mathrm{MeOC}_{6} \mathrm{H}_{4}\right)(20)$ & 70 & $9: 10: 26$ \\
\hline 25 & {$\left[\mathrm{Rh}(\mathrm{coe})_{2} \mathrm{Cl}\right]_{2}(5)$} & ${ }^{\mathrm{Me}} \mathrm{CgP}\left(3,5-\left(\mathrm{CF}_{3}\right)_{2} \mathrm{C}_{6} \mathrm{H}_{3}\right)(20)$ & 50 & $4: 24: 26$ \\
\hline 26 & {$\left[\mathrm{Rh}(\mathrm{coe})_{2} \mathrm{Cl}\right]_{2}(5)$} & ${ }^{\mathrm{Me}} \mathrm{CgP}\left(4-\mathrm{NCC}_{6} \mathrm{H}_{4}\right)(20)$ & 50 & $3: 42: 11$ \\
\hline 27 & {$\left[\mathrm{Rh}(\mathrm{coe})_{2} \mathrm{Cl}\right]_{2}(5)$} & ${ }^{\mathrm{Me}} \mathrm{CgP}\left(4-\left(\mathrm{EtO}_{2} \mathrm{C}\right) \mathrm{C}_{6} \mathrm{H}_{4}\right)(20)$ & 50 & $6: 27: 16$ \\
\hline 28 & {$[\mathrm{Rh}(\operatorname{cod}) \mathrm{Cl}]_{2}(5)$} & $\mathrm{AsPh}_{3}(20)$ & 50 & $8: 26: 15$ \\
\hline 29 & {$[\mathrm{Rh}(\operatorname{cod}) \mathrm{Cl}]_{2}(5)$} & $(p-\mathrm{Tol})_{3} \mathrm{As}(20)$ & 70 & $0: 34: 0$ \\
\hline 30 & {$[\mathrm{Rh}(\operatorname{cod}) \mathrm{Cl}]_{2}(5)$} & $\left(4-\mathrm{FC}_{6} \mathrm{H}_{4}\right)_{3} \mathrm{As}(20)$ & 70 & $11: 26: 13$ \\
\hline 31 & {$[\mathrm{Rh}(\operatorname{cod}) \mathrm{Cl}]_{2}(5)$} & $(\mathrm{PhO})_{3} \mathrm{P}(20)$ & 70 & $85: 0: 0$ \\
\hline 32 & {$[\mathrm{Rh}(\operatorname{cod}) \mathrm{Cl}]_{2}(5)$} & $\left(4-\mathrm{CF}_{3} \mathrm{C}_{6} \mathrm{H}_{4} \mathrm{O}\right)_{3} \mathrm{P}(20)$ & 70 & $7: 8: 20$ \\
\hline 33 & {$[\mathrm{Rh}(\operatorname{cod}) \mathrm{Cl}]_{2}(5)$} & $\left(\mathrm{CF}_{3} \mathrm{CH}_{2} \mathrm{O}\right)_{3} \mathrm{P}(20)$ & 70 & $84: 0: 0$ \\
\hline 34 & {$[\mathrm{Rh}(\operatorname{cod}) \mathrm{Cl}]_{2}(5)$} & BiPhePhos (20) & 70 & $0: 36: 3$ \\
\hline 35 & {$[\mathrm{Rh}(\operatorname{cod}) \mathrm{Cl}]_{2}(5)$} & MonoPhos (20) & 70 & $57: 17: 0$ \\
\hline 36 & {$[\mathrm{Rh}(\operatorname{cod}) \mathrm{Cl}]_{2}(5)$} & PipPhos (20) & 70 & $72: 7: 4$ \\
\hline
\end{tabular}

[a] Yields were determined by $1 \mathrm{H}$ NMR analysis using 1,4-dinitrobenzene as an internal standard 


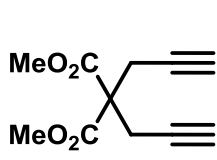

$1 \mathrm{a}$<smiles>O=C1C=CC(=O)C=C1</smiles>

$2 \mathbf{a}$

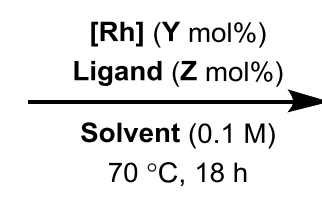

$70^{\circ} \mathrm{C}, 18 \mathrm{~h}$

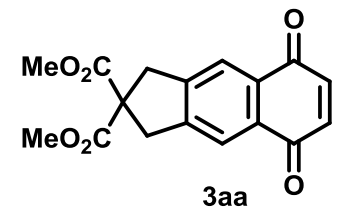

$\mathbf{X} \mathrm{mol} \%$

\begin{tabular}{|c|c|c|c|c|c|c|}
\hline Entry ${ }^{[a]}$ & $\mathbf{x}$ & Pre-catalyst (Y) & Ligand (Z) & Solvent & Oxidant (mol\%) & Yield $(\%)^{[b]}$ \\
\hline 1 & 100 & {$[\mathrm{Rh}(\operatorname{cod}) \mathrm{Cl}]_{2}(5)$} & BINAP (10) & THF & - & 27 \\
\hline 2 & 150 & {$[\mathrm{Rh}(\operatorname{cod}) \mathrm{Cl}]_{2}(5)$} & BINAP (10) & THF & - & 31 \\
\hline 3 & 200 & {$[\mathrm{Rh}(\operatorname{cod}) \mathrm{Cl}]_{2}(5)$} & BINAP (10) & THF & - & 44 \\
\hline 4 & 300 & {$[\mathrm{Rh}(\operatorname{cod}) \mathrm{Cl}]_{2}(5)$} & BINAP (10) & THF & - & 46 \\
\hline 5 & 200 & {$\left[\mathrm{Rh}(\mathrm{coe})_{2} \mathrm{Cl}\right]_{2}(3.75)$} & $\left(4-\mathrm{NCC}_{6} \mathrm{H}_{4}\right)_{3} \mathrm{P}(15)$ & DCE & - & 70 \\
\hline 6 & 100 & {$\left[\mathrm{Rh}(\mathrm{coe})_{2} \mathrm{Cl}\right]_{2}(3.75)$} & $\left(4-\mathrm{NCC}_{6} \mathrm{H}_{4}\right)_{3} \mathrm{P}(15)$ & DCE & - & 30 \\
\hline 7 & 100 & {$\left[\mathrm{Rh}(\mathrm{coe})_{2} \mathrm{Cl}\right]_{2}(5)$} & $\left(4-\mathrm{NCC}_{6} \mathrm{H}_{4}\right)_{3} \mathrm{P}(20)$ & DCE & DDQ (100) & no reaction \\
\hline 8 & 100 & {$\left[\mathrm{Rh}(\mathrm{coe})_{2} \mathrm{Cl}\right]_{2}(5)$} & $\left(4-\mathrm{NCC}_{6} \mathrm{H}_{4}\right)_{3} \mathrm{P}(20)$ & DCE & PIDA (100) & no reaction \\
\hline 9 & 100 & {$\left[\mathrm{Rh}(\mathrm{coe})_{2} \mathrm{Cl}\right]_{2}(3.75)$} & $\left(4-\mathrm{NCC}_{6} \mathrm{H}_{4}\right)_{3} \mathrm{P}(15)$ & DCE & $\mathrm{MnO}_{2}(300)$ & 48 \\
\hline
\end{tabular}

[a] General procedure A used for entries 1-4. General procedure B used for entries 5-9. [b] Yields were determined by ${ }^{1} \mathrm{H}$ NMR analysis using 1,4-dinitrobenzene as an internal standard 


\section{Ligand Synthesis}

\section{4,4',4'-Phosphanetriyltribenzonitrile}
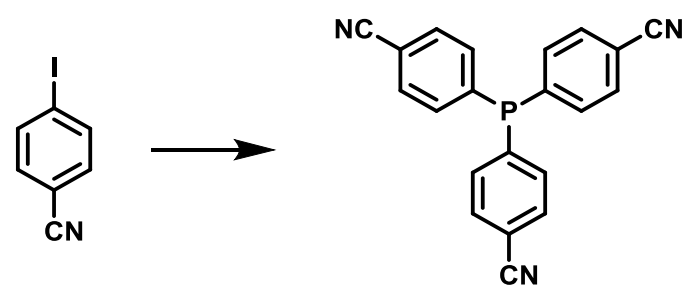

The title compound was prepared according to a modified literature procedure. ${ }^{1}$ To a solution of 4iodobenzonitrile $(4.00 \mathrm{~g}, 17.5 \mathrm{mmol})$ in $\mathrm{THF}(17.5 \mathrm{~mL})$ at $-40{ }^{\circ} \mathrm{C}$ was added $i-\mathrm{PrMgCl}(1.9 \mathrm{M}$ in THF, $9.7 \mathrm{~mL}, 18.4 \mathrm{mmol}$ ) dropwise over $5 \mathrm{~min}$. The solution was stirred between $-40{ }^{\circ} \mathrm{C}$ and $-30{ }^{\circ} \mathrm{C}$ for $40 \mathrm{~min}$, then a solution of $\mathrm{PCl}_{3}(0.51 \mathrm{~mL}, 5.82 \mathrm{mmol})$ in THF $(6 \mathrm{~mL})$ was added dropwise. The reaction mixture was warmed slowly to room temperature and stirred for $16 \mathrm{~h}$, then cooled to $0{ }^{\circ} \mathrm{C}$ and quenched by addition of $1 \mathrm{M}$ aq $\mathrm{HCl}(20 \mathrm{~mL})$. The aqueous phase was extracted with EtOAc $(3 \times 30 \mathrm{~mL})$ and the combined organic extracts were washed with brine $(20 \mathrm{~mL})$, dried over anhydrous $\mathrm{Na}_{2} \mathrm{SO}_{4}$ and concentrated in vacuo. Purification by flash column chromatography (20\%-50\% EtOAc/Hex) on silica gel afforded the title compound $(1.24 \mathrm{~g}, 63 \%)$ as a colourless solid; $\mathbf{R}_{f} 0.17$ (20\% EtOAc/Hex); mp $179-180{ }^{\circ} \mathrm{C}(\mathrm{EtOH})\left[\right.$ Lit., $\left.180-183{ }^{\circ} \mathrm{C}, \mathrm{EtOH}\right] ;{ }^{1} \mathbf{H} \mathbf{N M R}\left(400 \mathrm{MHz}, \mathrm{CDCl}_{3}\right) \delta 7.68-7.66(6 \mathrm{H}, \mathrm{m})$, 7.38-7.35 (6H, m) ${ }^{13} \mathbf{C}$ NMR $\left(101 \mathrm{MHz}, \mathrm{CDCl}_{3}\right) \delta 141.0\left(3 \times \mathrm{C}, \mathrm{d},{ }^{1} J_{\mathrm{CP}}=16.0 \mathrm{~Hz}\right), 134.2(6 \times \mathrm{CH}, \mathrm{d}$, $\left.{ }^{2} J_{\mathrm{CP}}=20.0 \mathrm{~Hz}\right), 132.6\left(6 \times \mathrm{CH}, \mathrm{d},{ }^{3} J_{\mathrm{CP}}=7.0 \mathrm{~Hz}\right), 118.2(3 \times \mathrm{C}), 113.8(3 \times \mathrm{C}) ;{ }^{31} \mathbf{P} \mathbf{N M R}(160 \mathrm{MHz}$, $\left.\mathrm{CDCl}_{3}\right): \delta-3.45$; The spectroscopic properties were consistent with the data available in the literature. ${ }^{2}$ 


\section{Substrate Synthesis}

Diyne $\mathbf{1 b}$ was purchased from a commercial source (TCI) and used as received. Diynes $\mathbf{1 \mathbf { a } ^ { 3 }}(72 \%), \mathbf{1 c}^{4}$ $(82 \%), \mathbf{1 d}^{5}(78 \%), \mathbf{1 e}^{6}(69 \%), \mathbf{1 g}^{7}(99 \%), \mathbf{1 i}^{8}$ (53\% over two steps), $\mathbf{1 j}^{6}(80 \%), \mathbf{1 k}^{9}$ (quant), $\mathbf{1 1}^{10}(90 \%)$, $\mathbf{1 n}^{11}$ (32\% over 3 steps) were synthesized following literature procedures. The spectroscopic properties of these compounds are consistent with the data available in the literature: $\mathbf{1 a}^{6}, \mathbf{1 c}^{4}, \mathbf{1 d}^{5}, \mathbf{1 e}^{6}, \mathbf{1 g}^{7}, \mathbf{1 i}^{8}$, $\mathbf{1 j}^{12}, \mathbf{1} \mathbf{k}^{9}, \mathbf{1 1}^{10}, \mathbf{1} \mathbf{n}^{11}$.

Quinones 2a, 2d and $\mathbf{2 l}$ were purchased from commercial sources (Aldrich) and purified by sublimation prior to use. Quinones $\mathbf{2} \mathbf{b}^{13}$ (92\%), $\mathbf{2 c}^{13}$ (80\%), $\mathbf{2 e}^{14}$ (49\%), $\mathbf{2 g}^{15}$ (69\%), $\mathbf{2 h}^{15}$ (96\%), $\mathbf{2 j}^{15}$ (76\%), $\mathbf{2 m}^{16}$ (73\% over two steps), $\mathbf{2} \mathbf{n}^{17}$ (52\%) and $\mathbf{2} \mathbf{o}^{18}$ (82\%) were synthesized following literature procedures. The spectroscopic properties of these compounds are consistent with the data available in the literature: $\mathbf{2} \mathbf{b}^{13}$, $\mathbf{2} \mathbf{c}^{13}, \mathbf{2} \mathbf{e}^{14}, \mathbf{2} \mathbf{g}^{19}, \mathbf{2} \mathbf{h}^{15}, \mathbf{2} \mathbf{j}^{15}, \mathbf{2 m}^{16}, \mathbf{2} \mathbf{n}^{20}, \mathbf{2 0}^{21}$.

(1f): $N, N$-Di(hept-2-yn-1-yl)-4-methylbenzenesulfonamide

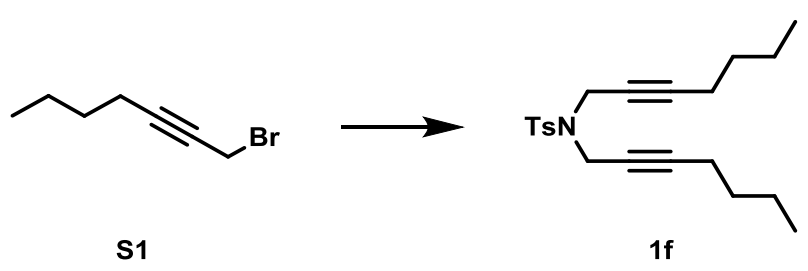

To a solution of bromide $\mathbf{S 1}^{22}(247 \mathrm{mg}, 1.41 \mathrm{mmol})$ in acetone $(6.4 \mathrm{~mL})$ was added $\mathrm{K}_{2} \mathrm{CO}_{3}(265 \mathrm{mg}$, $1.92 \mathrm{mmol})$ and $p$-toluenesulfonamide $(110 \mathrm{mg}, 0.641 \mathrm{mmol})$. The resulting suspension was stirred overnight for $18 \mathrm{~h}$, then cooled to room temperature and partitioned between $\mathrm{H}_{2} \mathrm{O}(10 \mathrm{~mL})$ and EtOAc $(10 \mathrm{~mL})$. The aqueous layer was extracted with EtOAc $(3 \times 5 \mathrm{~mL})$, then the combined organic layers were washed with brine $(5 \mathrm{~mL})$, dried over anhydrous $\mathrm{Na}_{2} \mathrm{SO}_{4}$ and concentrated in vacuo. Purification by flash column chromatography (10\% EtOAc/Hex) on silica gel afforded the title compound (173 mg, $74 \%$ ) as a colourless oil; $\mathbf{R}_{f} 0.62$ (20\% EtOAc/Hex); $\mathbf{v}_{\max } / \mathrm{cm}^{-1}: 2957$ (m), 2931 (m), 1431 (w), 1350 (s), 1161 (s), 1093 (s); ${ }^{1} \mathbf{H}$ NMR (400 MHz, CDCl $) \delta 7.71(2 \mathrm{H}, \mathrm{d}, J=8.5 \mathrm{~Hz}), 7.28(2 \mathrm{H}, \mathrm{d}, J=8.5 \mathrm{~Hz})$, $4.10(4 \mathrm{H}, \mathrm{t}, J=2.0 \mathrm{~Hz}), 2.41(3 \mathrm{H}, \mathrm{s}), 2.07-1.95(4 \mathrm{H}, \mathrm{m}), 1.40-1.21(8 \mathrm{H}, \mathrm{m}), 0.86(6 \mathrm{H}, \mathrm{t}, J=7.0 \mathrm{~Hz})$; ${ }^{13}$ C NMR $\left(101 \mathrm{MHz}, \mathrm{CDCl}_{3}\right) \delta 143.5(\mathrm{C}), 135.8(\mathrm{C}), 129.4(2 \times \mathrm{CH}), 128.1(2 \times \mathrm{CH}), 86.3(2 \times \mathrm{C})$, $72.5(2 \times \mathrm{C}), 36.8\left(2 \times \mathrm{CH}_{2}\right), 30.6\left(2 \times \mathrm{CH}_{2}\right), 22.0\left(2 \times \mathrm{CH}_{2}\right), 21.7\left(\mathrm{CH}_{3}\right), 18.4\left(2 \times \mathrm{CH}_{2}\right), 13.7(2 \times$ $\left.\mathrm{CH}_{3}\right) ;$ HRMS (ESI ${ }^{+}$): Calculated for $\mathrm{C}_{21} \mathrm{H}_{30} \mathrm{NO}_{2} \mathrm{~S}: 360.1992$. Found $[\mathrm{M}+\mathrm{H}]^{+}: 360.2003$. 


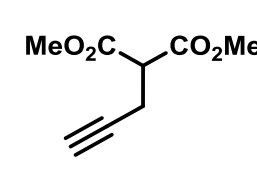

S2

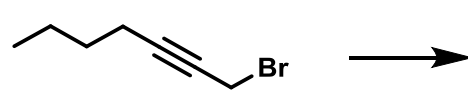

s1

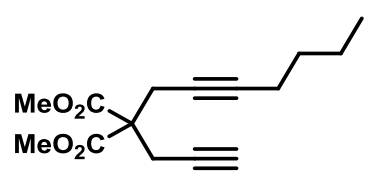

1h

To a solution of propargylmalonate $\mathbf{S 2}^{7}(300 \mathrm{mg}, 1.76 \mathrm{mmol})$ and bromide $\mathbf{S 1}^{22}(617 \mathrm{mg}, 3.53 \mathrm{mmol})$ in acetone $(3.5 \mathrm{~mL})$ was added $\mathrm{K}_{2} \mathrm{CO}_{3}(536 \mathrm{mg}, 3.88 \mathrm{mmol})$, and the resulting suspension was heated under reflux for $48 \mathrm{~h}$. The reaction mixture was cooled to room temperature and partitioned between $\mathrm{H}_{2} \mathrm{O}(10 \mathrm{~mL})$ and EtOAc $(10 \mathrm{~mL})$. The aqueous layer was extracted with EtOAc $(2 \times 10 \mathrm{~mL})$, then the combined organic layers were washed with brine $(5 \mathrm{~mL})$, dried over anhydrous $\mathrm{Na}_{2} \mathrm{SO}_{4}$ and concentrated in vacuo. Purification by flash column chromatography $(10 \% \mathrm{EtOAc} / \mathrm{Hex})$ on silica gel afforded the title compound (368 mg, 79\%) as a colourless oil; $\mathbf{R}_{f} 0.52\left(20 \%\right.$ EtOAc/Hex); ${ }^{1} \mathbf{H}$ NMR $\left(400 \mathrm{MHz}, \mathrm{CDCl}_{3}\right) \delta 3.74(6 \mathrm{H}, \mathrm{s}), 2.96(2 \mathrm{H}, \mathrm{d}, J=2.5 \mathrm{~Hz}), 2.93(2 \mathrm{H}, \mathrm{t}, J=2.5 \mathrm{~Hz}), 2.11(2 \mathrm{H}, \mathrm{tt}, J=$ 7.0, $2.5 \mathrm{~Hz}), 2.01(1 \mathrm{H}, \mathrm{t}, J=2.5 \mathrm{~Hz}), 1.46-1.30(4 \mathrm{H}, \mathrm{m}), 0.88(3 \mathrm{H}, \mathrm{t}, J=7.0 \mathrm{~Hz}) ;{ }^{13} \mathbf{C}$ NMR $(101 \mathrm{MHz}$, $\left.\mathrm{CDCl}_{3}\right) \delta 169.5(2 \times \mathrm{C}), 84.1(\mathrm{C}), 78.8(\mathrm{C}), 73.8(\mathrm{C}), 71.6(\mathrm{CH}), 57.0(\mathrm{C}), 53.1\left(2 \times \mathrm{CH}_{3}\right), 31.0\left(\mathrm{CH}_{2}\right)$, $23.2\left(\mathrm{CH}_{2}\right), 22.8\left(\mathrm{CH}_{2}\right), 21.9\left(\mathrm{CH}_{2}\right), 18.4\left(\mathrm{CH}_{2}\right), 13.7\left(\mathrm{CH}_{3}\right)$; The spectroscopic properties were consistent with the data available in the literature. ${ }^{23}$

(1m): $N$-Ethynyl-4-methyl- $N$-(2-(phenylethynyl)phenyl)benzenesulfonamide

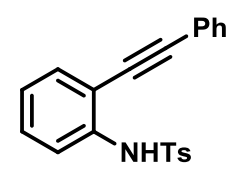

S3

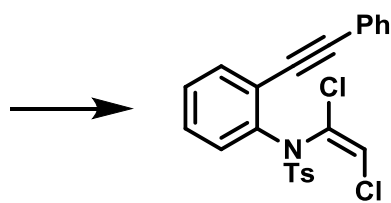

S4

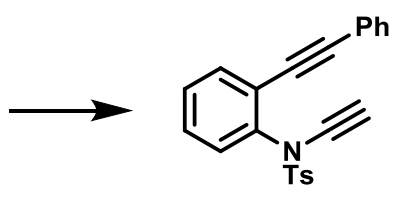

$1 \mathrm{~m}$

To a suspension of $N$-tosylaniline $\mathbf{S 3}^{24}(500 \mathrm{mg}, 1.44 \mathrm{mmol})$ and $\mathrm{K}_{2} \mathrm{CO}_{3}(597 \mathrm{mg}, 4.32 \mathrm{mmol})$ in DMF $(1.2 \mathrm{~mL})$ at room temperature was added trichloroethylene $(0.39 \mathrm{~mL}, 4.32 \mathrm{mmol})$. The resultant mixture was stirred at $100^{\circ} \mathrm{C}$ for $18 \mathrm{~h}$, then cooled to room temperature and partitioned between EtOAc $(60 \mathrm{~mL})$ and $\mathrm{H}_{2} \mathrm{O}(60 \mathrm{~mL})$. The aqueous phase was extracted with EtOAc $(20 \mathrm{~mL})$, then the combined organic phases were washed with brine $(3 \times 20 \mathrm{~mL})$, dried over anhydrous $\mathrm{Na}_{2} \mathrm{SO}_{4}$ and concentrated in vacuo. Purification by flash column chromatography $(10 \%$ EtOAc/Hex $)$ on silica gel afforded dichlorovinylsulfonamide $\mathbf{S 4}$ (335 mg, 53\%) as a pale yellow foam; $\mathbf{R}_{f} 0.32$ (20\% EtOAc/Hex); $\mathbf{v}_{\max } /$ $\mathrm{cm}^{-1}: 2988$ (w), 1598 (m), 1495 (m), 1365 (s), 1167 (s), 1078 (s); ${ }^{1} \mathbf{H}$ NMR (400 MHz, CDCl $\left.{ }_{3}\right) \delta 7.72$ $(2 \mathrm{H}, \mathrm{d}, J=8.5 \mathrm{~Hz}), 7.70-7.63(1 \mathrm{H}, \mathrm{m}), 7.60-7.55(1 \mathrm{H}, \mathrm{m}), 7.42-7.34(4 \mathrm{H}, \mathrm{m}), 7.34-7.28(3 \mathrm{H}, \mathrm{m}), 7.17$ $(2 \mathrm{H}, \mathrm{d}, J=8.0 \mathrm{~Hz}), 6.40(1 \mathrm{H}, \mathrm{s}), 2.30(3 \mathrm{H}, \mathrm{s}) ;{ }^{13} \mathrm{C}$ NMR $\left(101 \mathrm{MHz}, \mathrm{CDCl}_{3}\right) \delta 144.6(\mathrm{C}), 137.9(\mathrm{C})$, $136.1(\mathrm{C}), 134.3(\mathrm{CH}), 132.5(\mathrm{CH}), 131.6(2 \times \mathrm{CH}), 130.5(\mathrm{C}), 129.5(2 \times \mathrm{CH}), 129.4(\mathrm{CH}), 128.8(2 \times$ 
$\mathrm{CH}), 128.7(\mathrm{CH}), 128.5(\mathrm{CH}), 128.2(2 \times \mathrm{CH}), 125.0(\mathrm{C}), 123.1(\mathrm{C}), 119.2(\mathrm{CH}), 94.4(\mathrm{C}), 86.9(\mathrm{C})$, 21.6 $\left(\mathrm{CH}_{3}\right)$; HRMS $\left(\mathrm{ESI}^{+}\right)$: Calculated for $\mathrm{C}_{23} \mathrm{H}_{21} \mathrm{~N}_{2} \mathrm{O}_{2} \mathrm{Cl}_{2} \mathrm{~S}: 459.0695$. Found $\left[\mathrm{M}+\mathrm{NH}_{4}\right]^{+}: 459.0694$.

To a solution of dichlorovinylsulfonamide $\mathbf{S} 4(223 \mathrm{mg}, 0.504 \mathrm{mmol})$ in $\mathrm{THF}(5.0 \mathrm{~mL})$ at $-78{ }^{\circ} \mathrm{C}$ was added $\mathrm{PhLi}\left(1.9 \mathrm{M}\right.$ in $\mathrm{Bu}_{2} \mathrm{O}, 0.58 \mathrm{~mL}, 1.11 \mathrm{mmol}$ ) over $10 \mathrm{~min}$. The reaction was stirred at this temperature for $4 \mathrm{~h}$, then quenched with $\mathrm{H}_{2} \mathrm{O}-\mathrm{Et}_{2} \mathrm{O}(1: 1,0.5 \mathrm{~mL})$ and warmed to room temperature. The mixture was partitioned between sat aq $\mathrm{NH}_{4} \mathrm{Cl}(5 \mathrm{~mL})$ and $\mathrm{EtOAc}(5 \mathrm{~mL})$, then the aqueous phase was extracted with EtOAc $(2 \times 5 \mathrm{~mL})$. The combined organic phases were washed with brine $(5 \mathrm{~mL})$, dried over anhydrous $\mathrm{Na}_{2} \mathrm{SO}_{4}$ and concentrated in vacuo. Purification by flash column chromatography (20\% EtOAc/Hex) afforded the title compound (169 mg, 90\%) as an orange oil that solidified on standing; $\mathbf{R}_{f}$ 0.34 (20\% EtOAc/Hex); mp $130-132{ }^{\circ} \mathrm{C}\left(\mathrm{CH}_{2} \mathrm{Cl}_{2} /\right.$ petrol) [Lit., $132-133{ }^{\circ} \mathrm{C}$, pentane/ $\left.\mathrm{CHCl}_{3}\right] ;{ }^{1} \mathbf{H} \mathbf{~ N M R}$ (400 MHz, $\left.\mathrm{CDCl}_{3}\right) \delta$ 7.73-7.68 (2H, m), 7.54-7.49 (1H, m), 7.45-7.40 (1H, m), 7.39-7.27 (7H, m), 7.12-7.06 (2H, m), $2.94(1 \mathrm{H}, \mathrm{s}), 2.18(3 \mathrm{H}, \mathrm{s}) ;{ }^{13} \mathrm{C} \mathbf{N M R}\left(101 \mathrm{MHz}, \mathrm{CDCl}_{3}\right) \delta 145.1(\mathrm{C}), 138.3(\mathrm{C})$, $134.5(\mathrm{C}), 133.4(\mathrm{CH}), 131.7(2 \times \mathrm{CH}), 130.0(\mathrm{CH}), 129.7(2 \times \mathrm{CH}), 129.3(\mathrm{CH}), 129.1(\mathrm{CH}), 128.6$ $(\mathrm{CH}), 128.4(2 \times \mathrm{CH}), 128.1(2 \times \mathrm{CH}), 122.9(\mathrm{C}), 122.8(\mathrm{C}), 95.5(\mathrm{C}), 84.9(\mathrm{C}), 76.0(\mathrm{C}), 59.2(\mathrm{CH})$, $21.6\left(\mathrm{CH}_{3}\right)$; The spectroscopic properties were consistent with the data available in the literature. ${ }^{25}$

\section{(2f): 2-Iodo-1,4-benzoquinone}

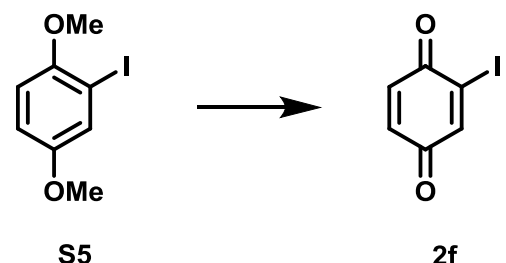

To stirring flash chromatographic-grade silica $(11.0 \mathrm{~g})$ was added a solution of $\left(\mathrm{NH}_{4}\right)_{2} \mathrm{Ce}\left(\mathrm{NO}_{3}\right)_{6}(5.01 \mathrm{~g}$, $9.14 \mathrm{mmol})$ in $\mathrm{H}_{2} \mathrm{O}(4.7 \mathrm{~mL})$ and stirring was continued until the mixture was free-flowing. $\mathrm{CH}_{2} \mathrm{Cl}_{2}$ (47 mL) was added, followed by a solution of iodide $\mathbf{S 5}^{26}(965 \mathrm{mg}, 3.65 \mathrm{mmol})$ in $\mathrm{CH}_{2} \mathrm{Cl}_{2}(4 \mathrm{~mL})$ and the resulting suspension was stirred for $30 \mathrm{~min}$. The reaction mixture was filtered through a pad of silica gel washing with $\mathrm{CH}_{2} \mathrm{Cl}_{2}(100 \mathrm{~mL})$ and the filtrate concentrated in vacuo. Purification by flash column chromatography (5\% EtOAc/Hex) on silica gel afforded the title compound (608 $\mathrm{mg}, 71 \%)$ as an orange solid; $\mathbf{R}_{f} 0.35$ (5\% EtOAc/Hex); mp 53-54 ${ }^{\circ} \mathrm{C}\left(\mathrm{CH}_{2} \mathrm{Cl}_{2} /\right.$ petrol) [Lit., 59-61 ${ }^{\circ} \mathrm{C}$, ligroin]; ${ }^{1} \mathbf{H}$ NMR $\left(400 \mathrm{MHz}, \mathrm{CDCl}_{3}\right) \delta 7.67(1 \mathrm{H}, \mathrm{d}, J=2.5 \mathrm{~Hz}), 7.00(1 \mathrm{H}, \mathrm{d}, J=10.0 \mathrm{~Hz}), 6.84(1 \mathrm{H}, \mathrm{dd}, J=10.0,2.5 \mathrm{~Hz})$; ${ }^{13}$ C NMR (101 MHz, CDCl $) \delta 184.2(\mathrm{C}), 180.3(\mathrm{C}), 146.3(\mathrm{CH}), 136.8(\mathrm{CH}), 134.7(\mathrm{CH}), 119.7(\mathrm{C})$; The spectroscopic properties were consistent with the data available in the literature. ${ }^{27}$ 
<smiles>O=C1C=CC(=O)C=C1</smiles>

$2 \mathbf{a}$<smiles>OCOc1ccccc1Br</smiles>

$\longrightarrow$<smiles>O=C1C=CC(=O)C(c2ccccc2Br)=C1</smiles>

2i

The title compound was prepared according to a literature procedure. ${ }^{15}$ To a solution of $1,4-$ benzoquinone (2a) $(200 \mathrm{mg}, 1.85 \mathrm{mmol})$ in $\mathrm{CH}_{2} \mathrm{Cl}_{2}(9.25 \mathrm{~mL})$ at room temperature was added 2bromophenylboronic acid (557 mg, $2.78 \mathrm{mmol})$, followed by $\mathrm{H}_{2} \mathrm{O}(5.6 \mathrm{~mL})$, a stock solution of $\mathrm{AgNO}_{3}$ (0.1 $\mathrm{M}$ in $\left.\mathrm{H}_{2} \mathrm{O}, 3.7 \mathrm{~mL}, 0.370 \mathrm{mmol}\right)$, and $\mathrm{K}_{2} \mathrm{~S}_{2} \mathrm{O}_{8}(1.50 \mathrm{~g}, 5.55 \mathrm{mmol})$. The resultant biphasic mixture was stirred vigorously for $16 \mathrm{~h}$, then filtered through Celite ${ }^{\circledR}$. The filtrate was diluted with $\mathrm{CH}_{2} \mathrm{Cl}_{2}$ $(10 \mathrm{~mL})$ and washed with sat aq $\mathrm{NaHCO}_{3}(10 \mathrm{~mL})$. The aqueous phase was extracted with $\mathrm{CH}_{2} \mathrm{Cl}_{2}(3 \times$ $10 \mathrm{~mL})$ and the combined organic layers were washed with brine $(10 \mathrm{~mL})$, dried over anhydrous $\mathrm{Na}_{2} \mathrm{SO}_{4}$ and concentrated in vacuo. Purification by flash column chromatography $(12.5 \%-25 \% \mathrm{EtOAc} / \mathrm{Hex})$ on silica gel afforded the title compound (439 mg, 90\%) as a yellow-orange solid; $\mathbf{R}_{f} 0.37$ (20\% EtOAc/Hex); mp 93-95 ${ }^{\circ} \mathrm{C}\left(\mathrm{CH}_{2} \mathrm{Cl}_{2} /\right.$ petrol) [Lit., 98-99 $\left.{ }^{\circ} \mathrm{C}\right] ;{ }^{1} \mathbf{H} \mathbf{~ N M R}\left(400 \mathrm{MHz}, \mathrm{CDCl}_{3}\right) \delta 7.65(1 \mathrm{H}$, d, $J=8.0 \mathrm{~Hz}), 7.41-7.37(1 \mathrm{H}, \mathrm{m}), 7.35-7.27(1 \mathrm{H}, \mathrm{m}), 7.20(1 \mathrm{H}, \mathrm{d}, J=7.0 \mathrm{~Hz}), 6.90(1 \mathrm{H}, \mathrm{d}, J=10.0 \mathrm{~Hz})$, $6.86(1 \mathrm{H}, \mathrm{d}, J=10.5 \mathrm{~Hz}), 6.77(1 \mathrm{H}, \mathrm{s}) ;{ }^{13} \mathrm{C}$ NMR $\left(101 \mathrm{MHz}, \mathrm{CDCl}_{3}\right) \delta 187.5(\mathrm{C}), 185.2(\mathrm{C}), 147.8(\mathrm{C})$, $137.0(\mathrm{CH}), 136.6(\mathrm{CH}), 134.9(\mathrm{CH}), 134.7(\mathrm{C}), 133.1(\mathrm{CH}), 131.0(\mathrm{CH}), 130.7(\mathrm{CH}), 127.6(\mathrm{CH})$, $122.8(\mathrm{C})$; The spectroscopic properties were consistent with the data available in the literature. ${ }^{28}$

(2k): 2-(Methyl(phenyl)amino)-1,4-benzoquinone<smiles>O=C1C=CC(=O)C=C1</smiles>

2a<smiles>N#[W]Nc1ccccc1</smiles><smiles>CCCC</smiles><smiles>CN(C1=CC(=O)C=CC1=O)c1ccccc1</smiles>

2k

The title compound was prepared according to a literature procedure. ${ }^{29}$ To a solution of 1,4benzoquinone (2a) $(2.09 \mathrm{~g}, 19.3 \mathrm{mmol})$ in $\mathrm{H}_{2} \mathrm{O}(200 \mathrm{~mL})$ at $0{ }^{\circ} \mathrm{C}$ was added a solution of $\mathrm{N}$ methylaniline $(1.03 \mathrm{~mL}, 9.51 \mathrm{mmol})$ in $\mathrm{H}_{2} \mathrm{O}-\mathrm{AcOH}\left(75 \mathrm{~mL}\right.$ of $\mathrm{H}_{2} \mathrm{O}$, with the minimum amount of $\mathrm{AcOH}$ to effect dissolution). The resulting mixture was stirred at $0{ }^{\circ} \mathrm{C}$ for $15 \mathrm{~min}$, then the product was collected by vacuum filtration. Recrystallisation $\left(\mathrm{H}_{2} \mathrm{O}\right)$ afforded the title compound $(385 \mathrm{mg}, 19 \%)$ as a dark red solid; $\mathbf{R}_{f} 0.16\left(20 \%\right.$ EtOAc/Hex); mp $129-131{ }^{\circ} \mathrm{C}\left(\mathrm{H}_{2} \mathrm{O}\right) ;{ }^{1} \mathbf{H} \mathbf{N M R}\left(400 \mathrm{MHz}, \mathrm{CDCl}_{3}\right) \delta$ 7.41-7.32 (2H, m), 7.28-7.21 (1H, m), 7.12-7.03 (2H, m), $6.63(1 \mathrm{H}, \mathrm{dd}, J=10.0,2.5 \mathrm{~Hz}), 6.49(1 \mathrm{H}, \mathrm{d}$, $J=10.0 \mathrm{~Hz}), 5.84(1 \mathrm{H}, \mathrm{d}, J=2.5 \mathrm{~Hz}), 3.33(3 \mathrm{H}, \mathrm{s}) ;{ }^{13} \mathbf{C ~ N M R}\left(101 \mathrm{MHz}, \mathrm{CDCl}_{3}\right) \delta 186.3(\mathrm{C}), 184.1$ 
(C), $150.3(\mathrm{C}), 147.6(\mathrm{C}), 137.5(\mathrm{CH}), 134.9(\mathrm{CH}), 129.8(2 \times \mathrm{CH}), 126.6(\mathrm{CH}), 125.4(2 \times \mathrm{CH}), 109.4$ $(\mathrm{CH}), 43.0\left(\mathrm{CH}_{3}\right)$. 


\section{Reaction Scope}

(3aa): Dimethyl 5,8-dioxo-1,3,5,8-tetrahydro-2H-cyclopenta[b]naphthalene-2,2-dicarboxylate

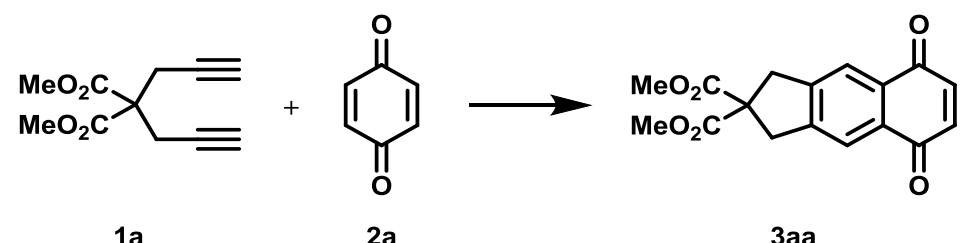

General Procedure B: Diyne 1a $(40.0 \mathrm{mg}, 0.192 \mathrm{mmol})$ and 1,4-benzoquinone (2a) $(41.6 \mathrm{mg}$, $0.385 \mathrm{mmol})$ were employed. Purification by flash column chromatography (15\% EtOAc/Hex) on silica gel afforded the title compound (42.4 mg, $70 \%$ ) as a yellow solid; $\mathbf{R}_{f} 0.50$ (33\% EtOAc/Hex); $\mathbf{m p} 112$ $114{ }^{\circ} \mathrm{C}\left(\mathrm{CH}_{2} \mathrm{Cl}_{2} / \mathrm{Hex}\right)$ [Lit., $115-117{ }^{\circ} \mathrm{C}, \mathrm{CH}_{2} \mathrm{Cl}_{2} / \mathrm{Hex}$ ]; ${ }^{1} \mathbf{H}$ NMR $\left(400 \mathrm{MHz}, \mathrm{CDCl}_{3}\right) \delta 7.92(2 \mathrm{H}, \mathrm{s})$, $6.93(2 \mathrm{H}, \mathrm{s}), 3.77(6 \mathrm{H}, \mathrm{s}), 3.70(4 \mathrm{H}, \mathrm{s}) ;{ }^{13} \mathrm{C}$ NMR $\left(101 \mathrm{MHz}, \mathrm{CDCl}_{3}\right) \delta 185.2(2 \times \mathrm{C}), 171.5(2 \times \mathrm{C})$, $146.9(2 \times \mathrm{C}), 138.7(2 \times \mathrm{CH}), 131.7(2 \times \mathrm{C}), 122.5(2 \times \mathrm{CH}), 60.2(\mathrm{C}), 53.4\left(2 \times \mathrm{CH}_{3}\right), 40.8\left(2 \times \mathrm{CH}_{2}\right)$; The spectroscopic properties were consistent with the data available in the literature. ${ }^{30}$

(3ab): Dimethyl 6,7-dimethyl-5,8-dioxo-1,3,5,8-tetrahydro-2H-cyclopenta[b]naphthalene-2,2dicarboxylate

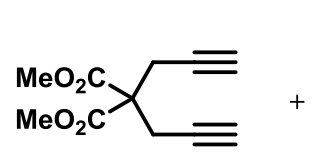

$1 \mathrm{a}$

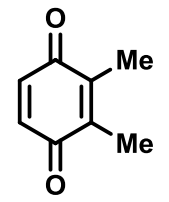

$2 b$

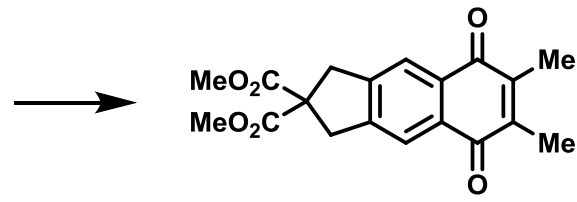

$3 a b$

General Procedure B: Diyne 1a (20.8 mg, $0.100 \mathrm{mmol})$ and benzoquinone $2 \mathbf{b}$ (27.3 mg, $0.200 \mathrm{mmol})$ were employed. Purification by flash column chromatography (25\% EtOAc/Hex) on silica gel afforded the title compound (31.2 mg, 91\%) as a pale yellow solid; $\mathbf{R}_{f} 0.44$ (33\% EtOAc/Hex); $\mathbf{m p} 162-164{ }^{\circ} \mathrm{C}$ $\left(\mathrm{CH}_{2} \mathrm{Cl}_{2} / \mathrm{Et}_{2} \mathrm{O}\right) ; \mathbf{v}_{\max } / \mathrm{cm}^{-1}: 2959$ (w), 1728 (s), 1654 (s), 1603 (m), 1427 (m), 1373 (m), 1265 (s), 1078 (s); ${ }^{1} \mathbf{H}$ NMR (400 MHz, CDCl $) \delta 7.89(2 \mathrm{H}, \mathrm{s}), 3.76(6 \mathrm{H}, \mathrm{s}), 3.68(4 \mathrm{H}, \mathrm{s}), 2.15(6 \mathrm{H}, \mathrm{s}) ;{ }^{13} \mathrm{C} \mathrm{NMR}$ $\left(101 \mathrm{MHz}, \mathrm{CDCl}_{3}\right) \delta 185.0(2 \times \mathrm{C}), 171.6(2 \times \mathrm{C}), 146.1(2 \times \mathrm{C}), 143.3(2 \times \mathrm{C}), 131.9(2 \times \mathrm{C}), 122.3$ $(2 \times \mathrm{CH}), 60.3(\mathrm{C}), 53.3\left(2 \times \mathrm{CH}_{3}\right), 40.7\left(2 \times \mathrm{CH}_{2}\right), 13.0\left(2 \times \mathrm{CH}_{3}\right)$; HRMS $\left(\mathrm{ESI}^{+}\right)$: Calculated for $\mathrm{C}_{19} \mathrm{H}_{19} \mathrm{O}_{6}: 343.1176$. Found $[\mathrm{M}+\mathrm{H}]^{+}: 343.1168$. 
(3ac): Dimethyl 5,8-dioxo-6-methoxy-1,3,5,8-tetrahydro-2H-cyclopenta[b]naphthalene-2,2dicarboxylate

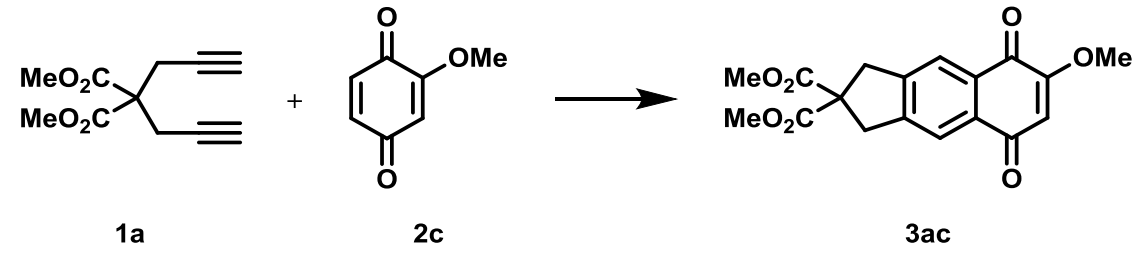

General Procedure B: Diyne 1a (40.0 mg, $0.192 \mathrm{mmol})$ and benzoquinone 2c (53.1 mg, $0.385 \mathrm{mmol})$ were employed. Purification by flash column chromatography (10\% acetone/PhMe) on silica gel afforded the title compound $(70.1 \mathrm{mg}, 78 \%)$ as a pale yellow solid; $\mathbf{R}_{f} 0.33$ (50\% EtOAc/Hex); $\mathbf{m p}$ 138-140 ${ }^{\circ} \mathrm{C}\left(\mathrm{CH}_{2} \mathrm{Cl}_{2} / \mathrm{Et}_{2} \mathrm{O}\right) ; \mathbf{v}_{\max } / \mathrm{cm}^{-1}: 2956$ (w), 1732 (s), 1681 (m), 1647 (m), 1610 (s),1435 (m), 1246 (s); ${ }^{1} \mathbf{H}$ NMR $\left(400 \mathrm{MHz}, \mathrm{CDCl}_{3}\right) \delta 7.92(1 \mathrm{H}, \mathrm{s}), 7.88(1 \mathrm{H}, \mathrm{s}), 6.10(1 \mathrm{H}, \mathrm{s}), 3.87(3 \mathrm{H}, \mathrm{s}), 3.75(6 \mathrm{H}$, s), 3.67 (4H, s); ${ }^{13}$ C NMR (100 MHz, $\left.\mathrm{CDCl}_{3}\right) \delta 184.9$ (C), 180.2 (C), 171.5 (2× C), 160.5 (C), 147.5 (C), $146.3(\mathrm{C}), 131.9(\mathrm{C}), 130.8(\mathrm{C}), 122.8(\mathrm{CH}), 122.3(\mathrm{CH}), 109.8(\mathrm{CH}), 60.3(\mathrm{C}), 56.6\left(\mathrm{CH}_{3}\right), 53.4$ $\left(2 \times \mathrm{CH}_{3}\right), 40.8\left(\mathrm{CH}_{2}\right), 40.6\left(\mathrm{CH}_{2}\right) ; \mathbf{H R M S}\left(\mathrm{ESI}^{+}\right)$: Calculated for $\mathrm{C}_{18} \mathrm{H}_{17} \mathrm{O}_{7}: 345.0969$. Found $[\mathrm{M}+\mathrm{H}]^{+}$: 345.0964 .

(3ad): Dimethyl 6-chloro-5,8-dioxo-1,3,5,8-tetrahydro-2H-cyclopenta[b]naphthalene-2,2dicarboxylate

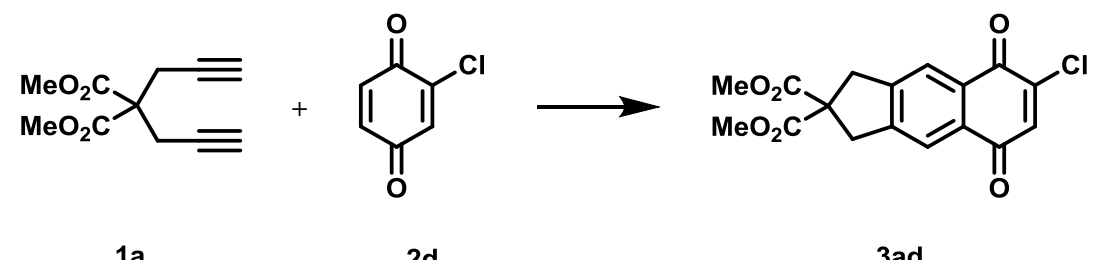

General Procedure B: Diyne 1a (40.0 mg, $0.192 \mathrm{mmol})$ and benzoquinone 2d (54.8 mg, $0.384 \mathrm{mmol})$ were employed. Purification by flash column chromatography $(14 \%-25 \%$ EtOAc/Hex $)$ on silica gel afforded the title compound (39.9 mg, 60\%) as a yellow solid; $\mathbf{R}_{f} 0.45$ (33\% EtOAc/Hex); mp $152-$ $155^{\circ} \mathrm{C}\left(\mathrm{CH}_{2} \mathrm{Cl}_{2} / \mathrm{Et}_{2} \mathrm{O}\right) ; \boldsymbol{v}_{\max } / \mathrm{cm}^{-1}: 2957$ (m), 1731 (s), 1678 (s), 1605 (s), 1570 (s), 1435 (m), 1281 (s); ${ }^{1} \mathbf{H}$ NMR $\left(400 \mathrm{MHz}, \mathrm{CDCl}_{3}\right) \delta 7.97(1 \mathrm{H}, \mathrm{s}), 7.89(1 \mathrm{H}, \mathrm{s}), 7.16(1 \mathrm{H}, \mathrm{s}), 3.76(6 \mathrm{H}, \mathrm{s}), 3.70(4 \mathrm{H}, \mathrm{br} \mathrm{s})$; ${ }^{13}$ C NMR (100 MHz, $\left.\mathrm{CDCl}_{3}\right) \delta 182.7$ (C), 178.0 (C), $171.3(2 \times \mathrm{C}), 147.7$ (C), 147.3 (C), 146.3 (C), $135.8(\mathrm{CH}), 131.5(\mathrm{C}), 131.0(\mathrm{C}), 123.6(\mathrm{CH}), 122.8(\mathrm{CH}), 60.2(\mathrm{C}), 53.4\left(2 \times \mathrm{CH}_{3}\right), 40.7\left(\mathrm{CH}_{2}\right), 40.6$ $\left(\mathrm{CH}_{2}\right)$; HRMS (ESI $\left.{ }^{+}\right)$: Calculated for $\mathrm{C}_{17} \mathrm{H}_{14} \mathrm{ClO}_{6}: 349.0473$. Found $[\mathrm{M}+\mathrm{H}]^{+}: 349.0479$. 
(3ae): Dimethyl 6-bromo-5,8-dioxo-1,3,5,8-tetrahydro-2H-cyclopenta[b]naphthalene-2,2dicarboxylate

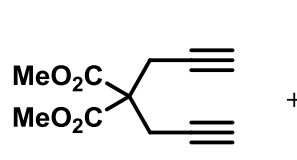

1a<smiles>O=C1C=CC(=O)C(Br)=C1</smiles>

$2 e$

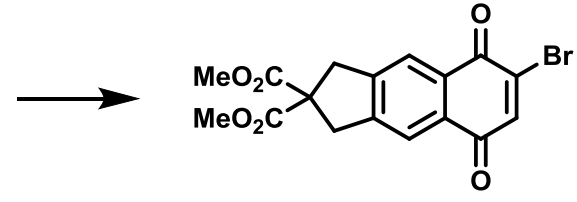

3ae

General Procedure B: Diyne 1a (40.0 mg, $0.192 \mathrm{mmol})$ and benzoquinone $2 \mathrm{e}(71.8 \mathrm{mg}, 0.385 \mathrm{mmol})$ were employed. Purification by flash column chromatography $\left(2 \% \mathrm{MeOH} / \mathrm{CH}_{2} \mathrm{Cl}_{2}\right)$ on silica gel afforded the title compound (24.2 $\mathrm{mg}, 32 \%)$ as a yellow solid; $\mathbf{R}_{\boldsymbol{f}} 0.43$ (33\% EtOAc/Hex); $\mathbf{m p} 162-$ $163{ }^{\circ} \mathrm{C}\left(\mathrm{CH}_{2} \mathrm{Cl}_{2} / \mathrm{Et}_{2} \mathrm{O}\right) ; \mathbf{v}_{\max } / \mathrm{cm}^{-1}: 2956$ (w), 1732 (s), 1677 (s), 1658 (s), 1605 (m), 1434 (m), 1255 (s); ${ }^{1} \mathrm{H}$ NMR (400 MHz, $\left.\mathrm{CDCl}_{3}\right) \delta 7.99(1 \mathrm{H}, \mathrm{s}), 7.90(1 \mathrm{H}, \mathrm{s}), 7.46$ (1H, s), 3.77 (6H, s), 3.70 (4H, br s); ${ }^{13}$ C NMR (101 MHz, $\left.\mathrm{CDCl}_{3}\right) \delta 182.5(\mathrm{C}), 177.9(\mathrm{C}), 171.4(2 \times \mathrm{C}), 147.6(\mathrm{C}), 147.3(\mathrm{C}), 140.3(\mathrm{CH})$, $140.1(\mathrm{C}), 131.5(\mathrm{C}), 130.7(\mathrm{C}), 123.9(\mathrm{CH}), 122.9(\mathrm{CH}), 60.2(\mathrm{C}), 53.4\left(2 \times \mathrm{CH}_{3}\right), 40.73\left(\mathrm{CH}_{2}\right), 40.67$ $\left(\mathrm{CH}_{2}\right)$; HRMS (ESI $\left.{ }^{+}\right)$: Calculated for $\mathrm{C}_{17} \mathrm{H}_{14} \mathrm{BrO}_{6}: 392.9968$. Found $[\mathrm{M}+\mathrm{H}]^{+}: 392.9960$.

(3af): Dimethyl 6-iodo-5,8-dioxo-1,3,5,8-tetrahydro-2H-cyclopenta[b]naphthalene-2,2dicarboxylate

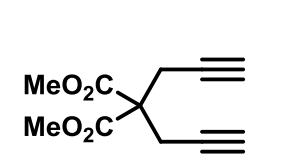

$1 \mathrm{a}$<smiles>O=C1C=CC(=O)C(I)=C1</smiles>

2f

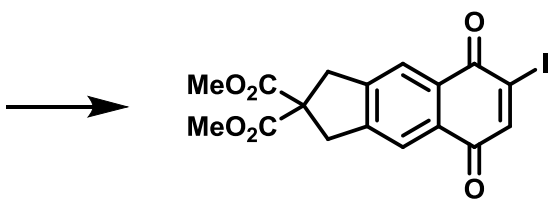

3af

General Procedure B: Diyne 1a (41.6 mg, $0.200 \mathrm{mmol}$ ) and benzoquinone $2 \mathbf{f}$ (93.6 mg, $0.400 \mathrm{mmol}$ ) were employed. Purification by flash column chromatography (25\% EtOAc/Hex) on silica gel afforded the title compound $(9.6 \mathrm{mg}, 22 \%)$ as a yellow solid; $\mathbf{R}_{\boldsymbol{f}} 0.50$ (33\% EtOAc/Hex); mp $148-150{ }^{\circ} \mathrm{C}$ $\left(\mathrm{CH}_{2} \mathrm{Cl}_{2} / \mathrm{Et}_{2} \mathrm{O}\right)$; $\boldsymbol{v}_{\max } / \mathrm{cm}^{-1}: 2955$ (w), 1732 (s), 1670 (s), 1606 (m), 1434 (m), 1279 (s), 1258 (s); ${ }^{1} \mathbf{H}$ NMR $\left(400 \mathrm{MHz}, \mathrm{CDCl}_{3}\right) \delta 7.98(1 \mathrm{H}, \mathrm{s}), 7.89(1 \mathrm{H}, \mathrm{s}), 7.84(1 \mathrm{H}, \mathrm{s}), 3.76(6 \mathrm{H}, \mathrm{s}), 3.70(4 \mathrm{H}, \mathrm{br})$; ${ }^{13}$ C NMR (101 MHz, $\left.\mathrm{CDCl}_{3}\right) \delta 182.1(\mathrm{C}), 178.8(\mathrm{C}), 171.4(2 \times \mathrm{C}), 148.4(\mathrm{CH}), 147.5(\mathrm{C}), 147.1(\mathrm{C})$, $131.5(\mathrm{C}), 129.5(\mathrm{C}), 124.3(\mathrm{CH}), 123.06(\mathrm{CH}), 123.04(\mathrm{C}), 60.2(\mathrm{C}), 53.4\left(2 \times \mathrm{CH}_{3}\right), 40.74\left(\mathrm{CH}_{2}\right)$, $40.67\left(\mathrm{CH}_{2}\right)$; HRMS $\left(\mathrm{ESI}^{+}\right)$: Calculated for $\mathrm{C}_{17} \mathrm{H}_{14} \mathrm{O}_{6} \mathrm{I}: 440.9830$. Found $[\mathrm{M}+\mathrm{H}]^{+}: 440.9825$. 


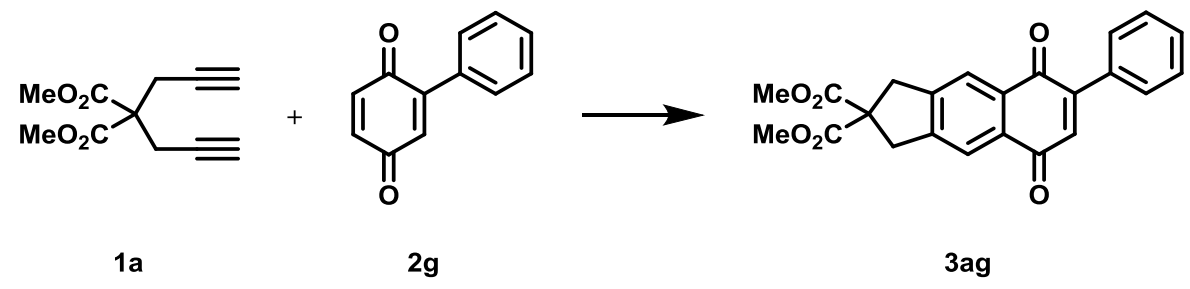

General Procedure B: Diyne 1a (40.0 mg, $0.192 \mathrm{mmol})$ and benzoquinone $2 \mathrm{~g}$ (70.8 mg, $0.384 \mathrm{mmol})$ were employed. Purification by flash column chromatography $(17 \%-25 \%$ EtOAc/Hex $)$ on silica gel afforded the title compound $(61.1 \mathrm{mg}, 81 \%)$ as a yellow solid; $\mathbf{R}_{f} 0.54$ (30\% EtOAc/Hex); $\mathbf{m p} 183-$ $185{ }^{\circ} \mathrm{C}\left(\mathrm{CH}_{2} \mathrm{Cl}_{2} / \mathrm{Et}_{2} \mathrm{O}\right) ; \mathbf{v}_{\max } / \mathrm{cm}^{-1}: 2956(\mathrm{~m}), 1732$ (s), 1665 (s), 1605 (s), 1434 (m), 1252 (s), 1180 (s), 1064 (s); ${ }^{1} \mathbf{H}$ NMR (400 MHz, $\left.\mathrm{CDCl}_{3}\right) \delta 8.01(1 \mathrm{H}, \mathrm{s}), 7.95(1 \mathrm{H}, \mathrm{s}), 7.56-7.54(2 \mathrm{H}, \mathrm{m}), 7.48-7.46$ (3H, m), 7.03 (1H, s), 3.77 (6H, s), 3.72 (4H, s); $\left.{ }^{13} \mathrm{C} \mathrm{NMR} \mathrm{(101} \mathrm{MHz,} \mathrm{CDCl}{ }_{3}\right) \delta 185.2(\mathrm{C}), 184.4(\mathrm{C}), 171.5$ $(2 \times \mathrm{C}), 148.1(\mathrm{C}), 146.9(\mathrm{C}), 146.8(\mathrm{C}), 135.2(\mathrm{CH}), 133.6(\mathrm{C}), 132.3(\mathrm{C}), 131.9(\mathrm{C}), 130.1(\mathrm{CH}), 129.6$ $(2 \times \mathrm{CH}), 128.6(2 \times \mathrm{CH}), 123.2(\mathrm{CH}), 122.0(\mathrm{CH}), 60.3(\mathrm{C}), 53.4\left(2 \times \mathrm{CH}_{3}\right), 40.79\left(\mathrm{CH}_{2}\right), 40.75\left(\mathrm{CH}_{2}\right)$; HRMS (ESI ${ }^{+}$: Calculated for $\mathrm{C}_{23} \mathrm{H}_{19} \mathrm{O}_{6}: 391.1182$. Found $[\mathrm{M}+\mathrm{H}]^{+}: 391.1168$.

(3ah): Dimethyl 6-(2-methoxyphenyl)-5,8-dioxo-1,3,5,8-tetrahydro-2Hcyclopenta[b]naphthalene-2,2-dicarboxylate

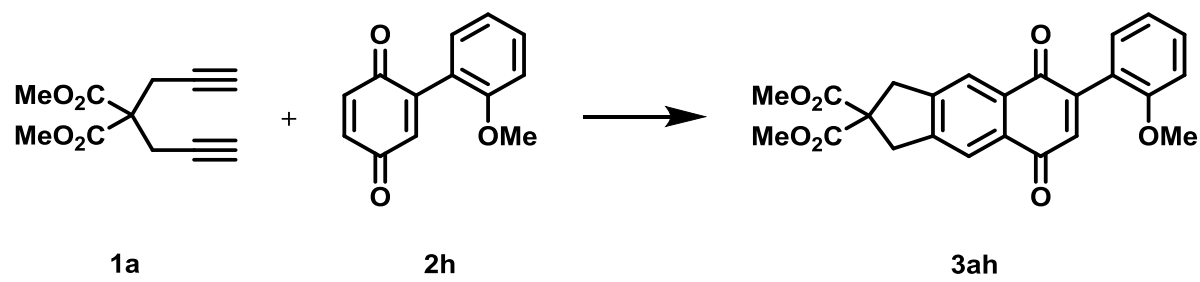

General Procedure B: Diyne 1a (40.0 mg, $0.192 \mathrm{mmol})$ and benzoquinone $2 \mathbf{h}(82.3 \mathrm{mg}, 0.384 \mathrm{mmol})$ were employed. Purification by flash column chromatography $(17 \%-33 \% \mathrm{EtOAc/Hex})$ on silica gel afforded the title compound (46.1 mg, 57\%) as a yellow foam; $\mathbf{R}_{f} 0.52$ (33\% EtOAc/Hex); mp 163$165{ }^{\circ} \mathrm{C}\left(\mathrm{CH}_{2} \mathrm{Cl}_{2} / \mathrm{Et}_{2} \mathrm{O}\right) ; \boldsymbol{v}_{\max } / \mathrm{cm}^{-1}: 2988$ (m), 1834 (s), 1668 (m), 1608 (m), 1435 (m), 1256 (s), 1066 (s); ${ }^{1} \mathrm{H}$ NMR $\left(400 \mathrm{MHz}, \mathrm{CDCl}_{3}\right) \delta 7.97(1 \mathrm{H}, \mathrm{s}), 7.95(1 \mathrm{H}, \mathrm{s}), 7.42(1 \mathrm{H}, \mathrm{td}, J=8.5,1.5 \mathrm{~Hz}), 7.22(1 \mathrm{H}$, dd, $J=7.5,1.5 \mathrm{~Hz}), 7.03(1 \mathrm{H}, \mathrm{t}, J=7.5 \mathrm{~Hz}), 6.98(1 \mathrm{H}, \mathrm{d}, J=8.5 \mathrm{~Hz}), 6.97(1 \mathrm{H}, \mathrm{s}), 3.78(3 \mathrm{H}, \mathrm{s}), 3.77$ $(6 \mathrm{H}, \mathrm{s}), 3.71(4 \mathrm{H}, \mathrm{s}) ;{ }^{13} \mathrm{C}$ NMR $\left(101 \mathrm{MHz}, \mathrm{CDCl}_{3}\right) \delta 185.4(\mathrm{C}), 183.6(\mathrm{C}), 171.5(2 \times \mathrm{C}), 157.4(\mathrm{C})$, $148.2(\mathrm{C}), 146.7(\mathrm{C}), 146.5(\mathrm{C}), 136.7(\mathrm{CH}), 132.4(\mathrm{C}), 132.0(\mathrm{C}), 131.2(\mathrm{CH}), 130.6(\mathrm{CH}), 123.6(\mathrm{C})$, $123.1(\mathrm{CH}), 122.1(\mathrm{CH}), 120.8(\mathrm{CH}), 111.4(\mathrm{CH}), 60.4(\mathrm{C}), 55.9\left(\mathrm{CH}_{3}\right), 53.4\left(2 \times \mathrm{CH}_{3}\right), 40.80\left(\mathrm{CH}_{2}\right)$, $40.76\left(\mathrm{CH}_{2}\right)$; HRMS (ESI $\left.{ }^{+}\right)$: Calculated for $\mathrm{C}_{24} \mathrm{H}_{21} \mathrm{O}_{7}: 421.1282$. Found $[\mathrm{M}+\mathrm{H}]^{+}: 421.1285$. 


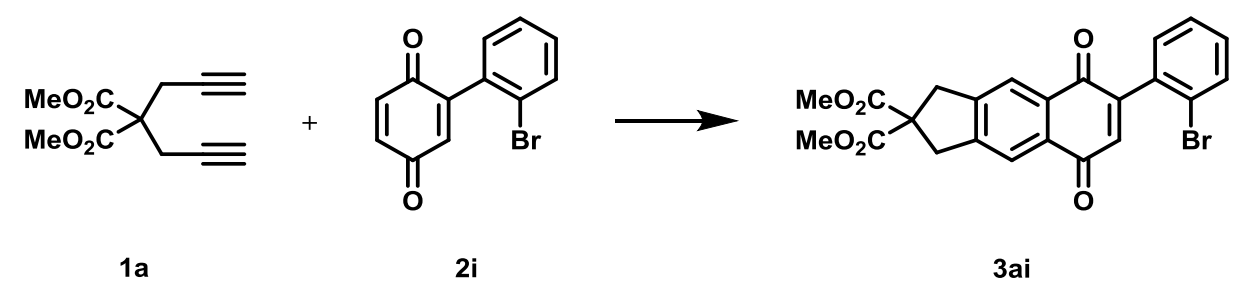

General Procedure B: Diyne 1a (40.0 mg, $0.192 \mathrm{mmol}$ ) and benzoquinone 2i (101.1 mg, $0.384 \mathrm{mmol}$ ) were employed. Purification by flash column chromatography (5\% acetone/ $\mathrm{PhMe})$ on silica gel afforded the title compound (50.2 $\mathrm{mg}, 56 \%)$ as a yellow solid; $\mathbf{R}_{f} 0.19(33 \% \mathrm{EtOAc} / \mathrm{Hex}) ; \mathbf{m p} 86-89{ }^{\circ} \mathrm{C}$ $\left(\mathrm{CH}_{2} \mathrm{Cl}_{2} / \mathrm{Et}_{2} \mathrm{O}\right) ; \mathbf{v}_{\max } / \mathrm{cm}^{-1}: 2988$ (w), 1732 (s), 1662 (s), 1606 (m), 1434 (m), 1285 (s), 1255 (s), 1067 (s); ${ }^{1} \mathbf{H}$ NMR (400 MHz, CDCl $) \delta 7.99(1 \mathrm{H}, \mathrm{s}), 7.97(1 \mathrm{H}, \mathrm{s}), 7.67(1 \mathrm{H}, \mathrm{dd}, J=8.0,1.0 \mathrm{~Hz}), 7.41(1 \mathrm{H}$, td, $J=7.5,1.0 \mathrm{~Hz}), 7.31(1 \mathrm{H}, \mathrm{td}, J=8.0,1.5 \mathrm{~Hz}), 7.26(1 \mathrm{H}, \mathrm{dd}, J=7.5,1.5 \mathrm{~Hz}), 6.92(1 \mathrm{H}, \mathrm{s}), 3.78(6 \mathrm{H}$, s), 3.73 (4H, s); ${ }^{13}$ C NMR (101 MHz, $\left.\mathrm{CDCl}_{3}\right) \delta 185.0$ (C), 183.2 (C), 171.5 (2× C), 149.7 (C), 147.1 (C), $147.0(\mathrm{C}), 137.1(\mathrm{CH}), 135.5(\mathrm{C}), 133.1(\mathrm{CH}), 132.0(\mathrm{C}), 131.9(\mathrm{C}), 130.8(\mathrm{CH}), 130.7(\mathrm{CH}), 127.5$ $(\mathrm{CH}), 123.2(\mathrm{CH}), 122.9(\mathrm{C}), 122.3(\mathrm{CH}), 60.3(\mathrm{C}), 53.4\left(2 \times \mathrm{CH}_{3}\right), 40.80\left(\mathrm{CH}_{2}\right), 40.79\left(\mathrm{CH}_{2}\right)$; HRMS $\left(\mathrm{ESI}^{+}\right)$: Calculated for $\mathrm{C}_{23} \mathrm{H}_{18} \mathrm{BrO}_{6}: 469.0281$. Found $[\mathrm{M}+\mathrm{H}]^{+}: 469.0264$.

(3aj): Dimethyl 5,8-dioxo-6-(4-(trifluoromethyl)phenyl)-1,3,5,8-tetrahydro-2Hcyclopenta $[b]$ naphthalene-2,2-dicarboxylate

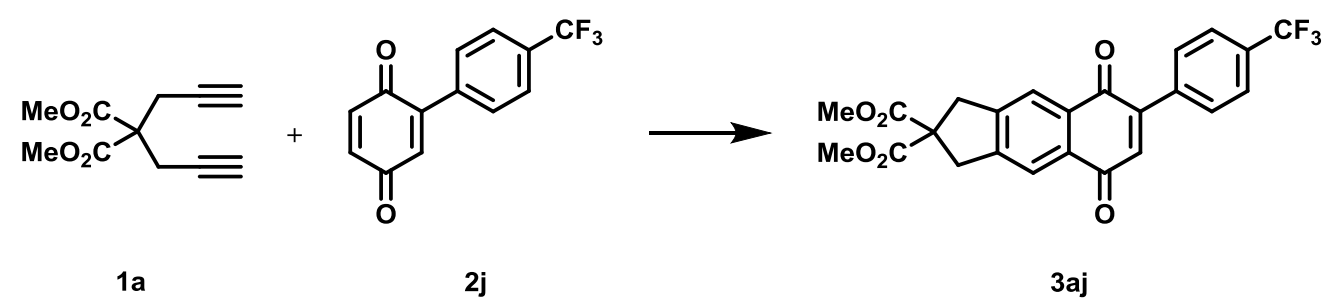

General Procedure B: Diyne 1a (40 mg, $0.192 \mathrm{mmol}$ ) and benzoquinone 2j (96.9 mg, $0.384 \mathrm{mmol}$ ) were employed. Purification by flash column chromatography $(12.5 \%-17 \% \mathrm{EtOAc} / \mathrm{Hex})$ on silica gel afforded the title compound (43.8 $\mathrm{mg}, 50 \%)$ as a yellow solid; $\mathbf{R}_{\boldsymbol{f}} 0.42$ (33\% EtOAc/Hex); mp $162-$ $164{ }^{\circ} \mathrm{C}\left(\mathrm{CH}_{2} \mathrm{Cl}_{2} / \mathrm{Et}_{2} \mathrm{O}\right) ; \mathbf{v}_{\max } / \mathrm{cm}^{-1}: 2956$ (w), 1732 (s), 1658 (s), 1605 (s), 1436 (m), 1323 (s), 1256 (s), $1164(\mathrm{~s}) ;{ }^{1} \mathbf{H}$ NMR $\left(400 \mathrm{MHz}, \mathrm{CDCl}_{3}\right) \delta 8.01(1 \mathrm{H}, \mathrm{s}), 7.95(1 \mathrm{H}, \mathrm{s}), 7.73(2 \mathrm{H}, \mathrm{d}, J=8.5 \mathrm{~Hz}), 7.66(2 \mathrm{H}$, d, $J=8.0 \mathrm{~Hz}), 7.05(1 \mathrm{H}, \mathrm{s}), 3.78(6 \mathrm{H}, \mathrm{s}), 3.73(4 \mathrm{H}, \mathrm{s}) ;{ }^{13} \mathbf{C ~ N M R}\left(101 \mathrm{MHz}, \mathrm{CDCl}_{3}\right) \delta 184.8(\mathrm{C}), 183.9$ (C), $171.4(2 \times$ C), $147.24(\mathrm{C}), 147.19(\mathrm{C}), 146.8(\mathrm{C}), 137.0(\mathrm{C}), 136.1(\mathrm{CH}) 132.0(\mathrm{C}), 131.8(\mathrm{C}), 129.9$ $(2 \times \mathrm{CH}), 125.5\left(2 \times \mathrm{CH}, \mathrm{q},{ }^{3} J_{\mathrm{CF}}=4.0 \mathrm{~Hz}\right), 123.2(\mathrm{CH}), 122.2(\mathrm{CH}), 60.3(\mathrm{C}), 53.4\left(2 \times \mathrm{CH}_{3}\right), 40.8$ $\left(\mathrm{CH}_{2}\right), 40.7\left(\mathrm{CH}_{2}\right)$, signals for $\underline{\mathrm{CF}}_{3}$ and $\underline{\mathrm{CCF}}_{3}$ were not observed; ${ }^{19} \mathbf{F} \mathbf{N M R}\left(376 \mathrm{MHz}, \mathrm{CDCl}_{3}\right) \delta-62.75$; HRMS (ESI ${ }^{+}$): Calculated for $\mathrm{C}_{24} \mathrm{H}_{18} \mathrm{~F}_{3} \mathrm{O}_{6}: 459.1050$. Found $[\mathrm{M}+\mathrm{H}]^{+}: 459.1051$. 
(3ak): Dimethyl 6-(methyl(phenyl)amino)-5,8-dioxo-1,3,5,8-tetrahydro-2H-

cyclopenta[b]naphthalene-2,2-dicarboxylate

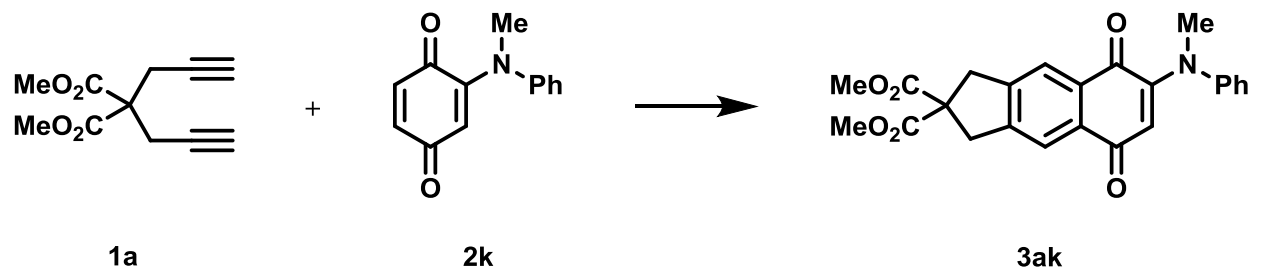

General Procedure B: Diyne 1a $(40.0 \mathrm{mg}, 0.192 \mathrm{mmol})$ and benzoquinone 2k (81.9 mg, $0.384 \mathrm{mmol})$ were employed. Purification by flash column chromatography ( $25 \%$ EtOAc/Hex) on silica gel afforded the title compound $(46.9 \mathrm{mg}, 58 \%)$ as a red solid; $\mathbf{R}_{f} 0.24(33 \% \mathrm{EtOAc} / \mathrm{Hex}) ; \mathbf{m p} 89-91{ }^{\circ} \mathrm{C}$ $\left(\mathrm{CH}_{2} \mathrm{Cl}_{2} / \mathrm{Et}_{2} \mathrm{O}\right) ; \boldsymbol{v}_{\max } / \mathrm{cm}^{-1}: 2901$ (m), 1733 (s), 1677 (m), 1605 (m), 1557 (s), 1282 (s); ${ }^{1} \mathbf{H}$ NMR $\left(400 \mathrm{MHz}, \mathrm{CDCl}_{3}\right) \delta 7.90(1 \mathrm{H}, \mathrm{s}), 7.70(1 \mathrm{H}, \mathrm{s}), 7.41-7.34(2 \mathrm{H}, \mathrm{m}), 7.27-7.23(1 \mathrm{H}, \mathrm{m}), 7.12-7.06(2 \mathrm{H}$, m), $6.08(1 \mathrm{H}, \mathrm{s}), 3.75(6 \mathrm{H}, \mathrm{s}), 3.67(2 \mathrm{H}, \mathrm{s}), 3.63(2 \mathrm{H}, \mathrm{s}), 3.38(3 \mathrm{H}, \mathrm{s}) ;{ }^{13} \mathrm{C} \mathrm{NMR}\left(101 \mathrm{MHz}, \mathrm{CDCl}_{3}\right) \delta$ $183.8(\mathrm{C}), 182.2(\mathrm{C}), 171.6(2 \times \mathrm{C}), 152.2(\mathrm{C}), 148.2$ (C), 146.8 (C), $145.2(\mathrm{C}), 132.5(\mathrm{C}), 132.2(\mathrm{C})$, $129.7(2 \times \mathrm{CH}), 126.4(\mathrm{CH}), 125.3(2 \times \mathrm{CH}), 122.8(\mathrm{CH}), 121.7(\mathrm{CH}), 111.9(\mathrm{CH}), 60.3(\mathrm{C}), 53.3(2 \times$ $\left.\mathrm{CH}_{3}\right), 43.3\left(\mathrm{CH}_{3}\right), 40.8\left(\mathrm{CH}_{2}\right), 40.6\left(\mathrm{CH}_{2}\right)$; HRMS (ESI $\left.{ }^{+}\right)$: Calculated for $\mathrm{C}_{24} \mathrm{H}_{22} \mathrm{NO}_{6}: 420.1442$. Found $[\mathrm{M}+\mathrm{H}]^{+}: 420.1445$.

(3al): Dimethyl 5,10-dioxo-1,3,5,10-tetrahydro-2H-cyclopenta[b]anthracene-2,2-dicarboxylate

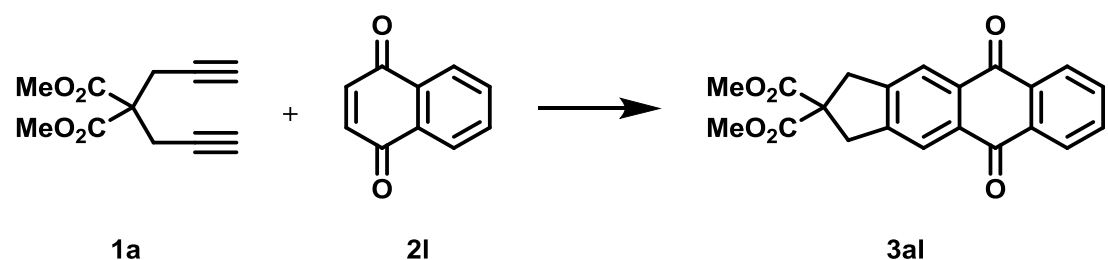

General Procedure B: Diyne 1a $(40.0 \mathrm{mg}, 0.192 \mathrm{mmol})$ and naphthoquinone 2l $(60.8 \mathrm{mg}$, $0.385 \mathrm{mmol})$ were employed. Purification by recrystallisation $(50 \%$ EtOAc/Hex) afforded the title compound $(42.4 \mathrm{mg}, 62 \%)$ as an off-white solid; $\mathbf{R}_{f} 0.38$ (33\% EtOAc/Hex); $\mathbf{m p ~} 244-246{ }^{\circ} \mathrm{C}$ (EtOAc/Hex); $\mathbf{v}_{\max } / \mathrm{cm}^{-1}: 2988$ (w), 1729 (s), 1674 (m), 1592 (m), 1394 (m), 1255 (s) 1066 (s); ${ }^{1} \mathbf{H}$ NMR $\left(400 \mathrm{MHz}, \mathrm{CDCl}_{3}\right) \delta 8.29(2 \mathrm{H}, \mathrm{dt}, J=7.0,3.5 \mathrm{~Hz}), 8.14(2 \mathrm{H}, \mathrm{s}), 7.79(2 \mathrm{H}, \mathrm{dd}, J=6.0,3.5 \mathrm{~Hz})$, $3.78(6 \mathrm{H}, \mathrm{s}), 3.74(4 \mathrm{H}, \mathrm{s}) ;{ }^{13} \mathrm{C}$ NMR $\left(101 \mathrm{MHz}, \mathrm{CDCl}_{3}\right) \delta 183.2(2 \times \mathrm{C}), 171.5(2 \times \mathrm{C}), 147.2(2 \times \mathrm{C})$, 134.2 $(2 \times \mathrm{CH}), 133.6(2 \times \mathrm{C}), 133.3(2 \times \mathrm{C}), 127.4(2 \times \mathrm{CH}), 123.3(2 \times \mathrm{CH}), 60.3(\mathrm{C}), 53.4\left(2 \times \mathrm{CH}_{3}\right)$, $40.8\left(2 \times \mathrm{CH}_{3}\right)$; HRMS $\left(\mathrm{ESI}^{+}\right)$: Calculated for $\mathrm{C}_{21} \mathrm{H}_{17} \mathrm{O}_{6}: 365.1020$. Found $[\mathrm{M}+\mathrm{H}]^{+}: 365.1019$. 


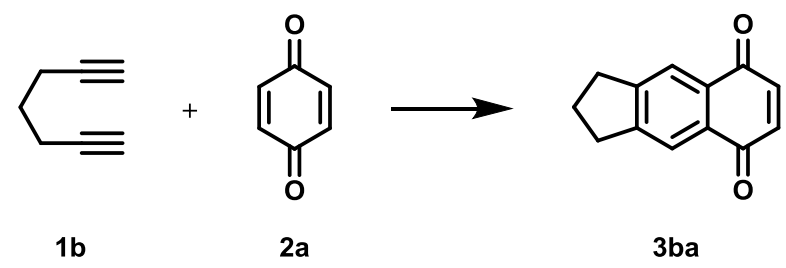

General Procedure B: 1,6-Heptadiyne (1b) $(26.6 \mathrm{mg}, 0.288 \mathrm{mmol})$ and 1,4-benzoquinone (2a) $(62.3 \mathrm{mg}, 0.576 \mathrm{mmol})$ were employed. Purification by flash column chromatography $(12.5 \%$ EtOAc/Hex) on silica gel afforded the title compound ( $28.3 \mathrm{mg}, 50 \%$ ) as a yellow solid; $\mathbf{R}_{f} 0.67$ (33\% EtOAc/Hex); mp 104-106 ${ }^{\circ} \mathrm{C}\left(\mathrm{CH}_{2} \mathrm{Cl}_{2} / \mathrm{Et}_{2} \mathrm{O}\right) ; \mathbf{v}_{\max } / \mathrm{cm}^{-1}: 2956$ (m), 1663 (s), 1601 (s), 1424 (m), 1319 (s), 1129 (s); ${ }^{1} \mathbf{H}$ NMR (400 MHz, CDCl $) \delta 7.89(2 \mathrm{H}, \mathrm{s}), 6.90(2 \mathrm{H}, \mathrm{s}), 3.02(4 \mathrm{H}, \mathrm{t}, J=7.5 \mathrm{~Hz}), 2.15$ $(2 \mathrm{H}, \mathrm{p}, J=7.5 \mathrm{~Hz}) ;{ }^{13} \mathbf{C} \mathbf{~ N M R}\left(101 \mathrm{MHz}, \mathrm{CDCl}_{3}\right) \delta 185.6(2 \times \mathrm{C}), 151.3(2 \times \mathrm{C}), 138.6(2 \times \mathrm{CH}), 131.0$ $(2 \times \mathrm{C}), 122.4(2 \times \mathrm{CH}), 33.2\left(2 \times \mathrm{CH}_{2}\right), 25.2\left(\mathrm{CH}_{2}\right)$; HRMS $\left(\mathrm{APCI}^{+}\right)$: Calculated for $\mathrm{C}_{13} \mathrm{H}_{11} \mathrm{O}_{2}$ : 199.0754. Found $[\mathrm{M}+\mathrm{H}]^{+}: 199.0746$.

(3ca): 2-Tosyl-2,3-dihydro-1H-benzo[f]isoindole-5,8-dione

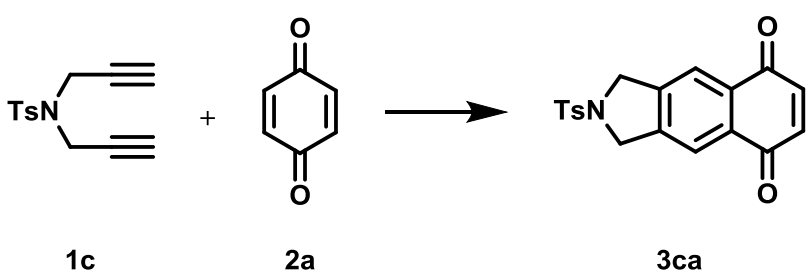

General Procedure B: Diyne 1c $(71.3 \mathrm{mg}, 0.288 \mathrm{mmol})$ and 1,4-benzoquinone (2a) $(62.3 \mathrm{mg}$, $0.576 \mathrm{mmol}$ ) were employed. Purification by flash column chromatography (33\% EtOAc/Hex - EtOAc neat) on silica gel afforded the title compound (102 mg, 66\%) as a pale brown solid; $\mathbf{R}_{f} 0.45$ (33\% EtOAc/Hex); mp 214-215 ${ }^{\circ} \mathrm{C}\left(\mathrm{CH}_{2} \mathrm{Cl}_{2} / \mathrm{Et}_{2} \mathrm{O}\right) ; \mathbf{v}_{\max } / \mathrm{cm}^{-1}: 2988$ (w), 1659 (s), 1613 (m), 1340 (s), 1158 (s), 1045 (s); ${ }^{1} \mathbf{H}$ NMR (400 MHz, $\left.\mathrm{CDCl}_{3}\right) \delta 7.89(2 \mathrm{H}, \mathrm{s}), 7.78(2 \mathrm{H}, \mathrm{d}, J=8.0 \mathrm{~Hz}), 7.34(2 \mathrm{H}, \mathrm{d}, J=$ $8.0 \mathrm{~Hz}), 6.96(2 \mathrm{H}, \mathrm{s}), 4.71(4 \mathrm{H}, \mathrm{s}), 2.40(3 \mathrm{H}, \mathrm{s}) ;{ }^{13} \mathbf{C ~ N M R}\left(101 \mathrm{MHz}, \mathrm{CDCl}_{3}\right) \delta 184.5(2 \times \mathrm{C}), 144.3$ (C), $142.7(2 \times \mathrm{C}), 138.7(2 \times \mathrm{CH}), 133.4(\mathrm{C}), 132.1(2 \times \mathrm{C}), 130.2(2 \times \mathrm{CH}), 127.7(2 \times \mathrm{CH}), 121.1(2$ $\times \mathrm{CH}), 53.7\left(2 \times \mathrm{CH}_{2}\right), 21.7\left(\mathrm{CH}_{3}\right) ; \mathbf{H R M S}\left(\mathrm{ESI}^{+}\right)$: Calculated for $\mathrm{C}_{19} \mathrm{H}_{16} \mathrm{NO}_{4} \mathrm{~S}: 354.0795$. Found [M + $\mathrm{H}]^{+}: 354.0787$. 


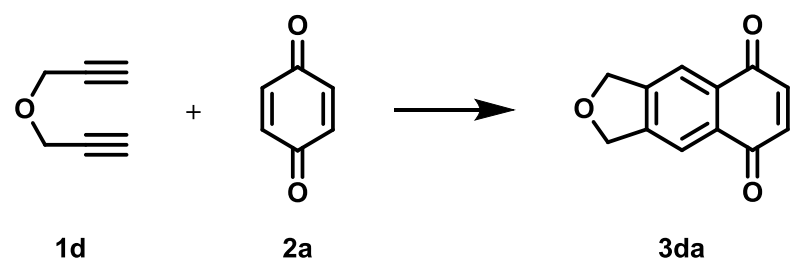

General Procedure B: Diyne 1d $(27.1 \mathrm{mg}, 0.288 \mathrm{mmol})$ and 1,4-benzoquinone (2a) (62.3 mg, $0.576 \mathrm{mmol}$ ) were employed. Purification by flash column chromatography (33\% EtOAc/Hex) on silica gel afforded the title compound $(26.5 \mathrm{mg}, 46 \%)$ as a yellow solid; $\mathbf{R}_{f} 0.49$ (33\% EtOAc/Hex); mp decomp. $>190{ }^{\circ} \mathrm{C} ; \mathbf{v}_{\max } / \mathrm{cm}^{-1}: 2918$ (s), 2850 (m), 1667 (s), 1603 (s), 1311 (s), 1045 (s); ${ }^{1} \mathbf{H}$ NMR $\left(400 \mathrm{MHz}, \mathrm{CDCl}_{3}\right) 7.95(2 \mathrm{H}, \mathrm{s}), 6.97(2 \mathrm{H}, \mathrm{s}), 5.19(4 \mathrm{H}, \mathrm{s}) ;{ }^{13} \mathbf{C} \mathbf{N M R}\left(101 \mathrm{MHz}, \mathrm{CDCl}_{3}\right) \delta 184.9(2 \times$ C), $145.7(2 \times \mathrm{C}), 138.7(2 \times \mathrm{CH}), 132.1(2 \times \mathrm{C}), 119.4(2 \times \mathrm{CH}), 73.4\left(2 \times \mathrm{CH}_{2}\right) ;$ HRMS $\left(\mathrm{ESI}^{+}\right)$: Calculated for $\mathrm{C}_{12} \mathrm{H}_{9} \mathrm{O}_{3}: 201.0546$. Found $[\mathrm{M}+\mathrm{H}]^{+}: 201.0553$.

(3ea): Dimethyl 4,9-dimethyl-5,8-dioxo-1,3,5,8-tetrahydro-2H-cyclopenta[b]naphthalene-2,2dicarboxylate

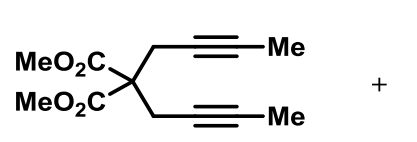

$1 \mathrm{e}$<smiles>O=C1C=CC(=O)C=C1</smiles>

2a

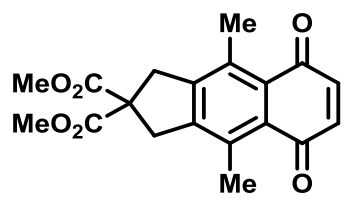

3ea

General Procedure C: Diyne 1e $(23.6 \mathrm{mg}, 0.100 \mathrm{mmol})$ and 1,4-benzoquinone (2a) $(54.0 \mathrm{mg}$, $0.500 \mathrm{mmol}$ ) were employed. Purification by flash column chromatography (5\% acetone/PhMe) on silica gel afforded the title compound $\mathbf{3 e a}(26.3 \mathrm{mg}, 77 \%)$ as a yellow solid; $\mathbf{R}_{f} 0.50$ (33\% EtOAc/Hex); mp 223-225 ${ }^{\circ} \mathrm{C}\left(\mathrm{CH}_{2} \mathrm{Cl}_{2} / \mathrm{Et}_{2} \mathrm{O}\right) ; \mathbf{v}_{\max } / \mathrm{cm}^{-1}: 2971$ (w), 1738 (s), 1652 (s), 1279 (s), 1083 (s); ${ }^{1} \mathbf{H}$ NMR $\left(400 \mathrm{MHz}, \mathrm{CDCl}_{3}\right) \delta 6.77(2 \mathrm{H}, \mathrm{s}), 3.78(6 \mathrm{H}, \mathrm{s}), 3.70(4 \mathrm{H}, \mathrm{s}), 2.59(6 \mathrm{H}, \mathrm{s}) ;{ }^{13} \mathbf{C} \mathbf{N M R}\left(101 \mathrm{MHz}, \mathrm{CDCl}_{3}\right)$ $\delta 188.1(2 \times \mathrm{C}), 171.8(2 \times \mathrm{C}), 146.1(2 \times \mathrm{C}), 138.6(2 \times \mathrm{CH}), 135.2(2 \times \mathrm{C}), 130.9(2 \times \mathrm{C}), 58.3(\mathrm{C})$, $53.4\left(2 \times \mathrm{CH}_{3}\right), 41.0\left(2 \times \mathrm{CH}_{2}\right), 18.7\left(2 \times \mathrm{CH}_{3}\right)$; HRMS $\left(\mathrm{ESI}^{+}\right)$: Calculated for $\mathrm{C}_{19} \mathrm{H}_{19} \mathrm{O}_{6}: 343.1176$. Found $[\mathrm{M}+\mathrm{H}]^{+}: 343.1173$. 


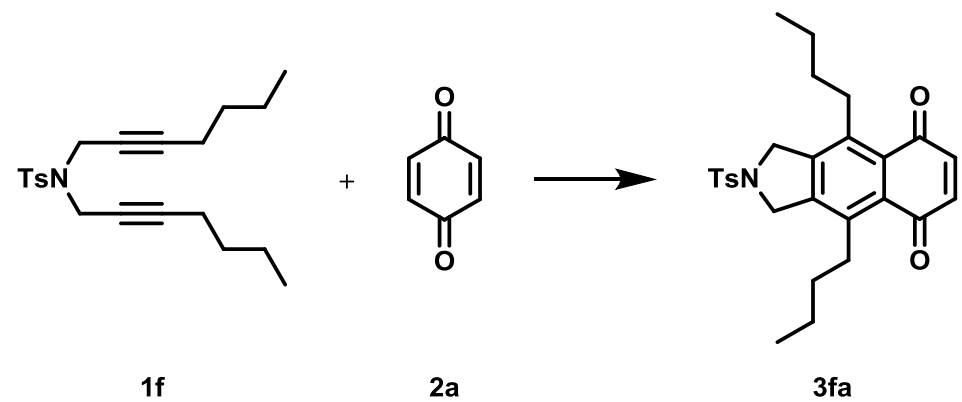

General Procedure C: Diyne 1f $(36.0 \mathrm{mg}, 0.100 \mathrm{mmol})$ and 1,4-benzoquinone (2a) $(54.0 \mathrm{mg}$, $0.500 \mathrm{mmol})$ were employed. Purification by flash column chromatography (15\% EtOAc/Hex) on silica gel afforded the title compound (32.1 mg, 69\%) as yellow solid; $\mathbf{R}_{f} 0.80$ (33\% EtOAc/Hex); mp 166$168{ }^{\circ} \mathrm{C}\left(\mathrm{CH}_{2} \mathrm{Cl}_{2} / \mathrm{Et}_{2} \mathrm{O}\right) ; \mathbf{v}_{\max } / \mathrm{cm}^{-1}: 2968$ (w), 1663 (s), $1612(\mathrm{~m}), 1347$ (s), 1265 (s), 1162 (s); ${ }^{1} \mathbf{H}$ NMR $\left(400 \mathrm{MHz}, \mathrm{CDCl}_{3}\right) \delta 7.80(2 \mathrm{H}, \mathrm{d}, J=8.0 \mathrm{~Hz}), 7.34(2 \mathrm{H}, \mathrm{d}, J=8.0 \mathrm{~Hz}), 6.77(2 \mathrm{H}, \mathrm{s}), 4.73(4 \mathrm{H}, \mathrm{s}), 2.92-$ $2.84(4 \mathrm{H}, \mathrm{m}), 2.41(3 \mathrm{H}, \mathrm{s}), 1.52-1.39(8 \mathrm{H}, \mathrm{m}), 0.97(6 \mathrm{H}, \mathrm{t}, J=7.0 \mathrm{~Hz}) ;{ }^{13} \mathbf{C} \mathbf{N M R}\left(101 \mathrm{MHz}, \mathrm{CDCl}_{3}\right)$ $\delta 187.4(2 \times \mathrm{C}), 144.2(\mathrm{C}), 142.3(2 \times \mathrm{C}), 139.2(2 \times \mathrm{C}), 138.6(2 \times \mathrm{CH}), 133.7(\mathrm{C}), 131.1(2 \times \mathrm{C}), 130.2$ $(2 \times \mathrm{CH}), 127.6(2 \times \mathrm{CH}), 54.0\left(2 \times \mathrm{CH}_{2}\right), 31.8\left(2 \times \mathrm{CH}_{2}\right), 31.7\left(2 \times \mathrm{CH}_{2}\right), 23.5\left(2 \times \mathrm{CH}_{2}\right), 21.7\left(\mathrm{CH}_{3}\right)$, $14.0\left(2 \times \mathrm{CH}_{3}\right)$; HRMS $\left(\mathrm{ESI}^{+}\right)$: Calculated for $\mathrm{C}_{27} \mathrm{H}_{32} \mathrm{NO}_{4} \mathrm{~S}: 466.2047$. Found $[\mathrm{M}+\mathrm{H}]^{+}: 466.2059$.

(3ga): Dimethyl 4-methyl-5,8-dioxo-1,3,5,8-tetrahydro-2H-cyclopenta[b]naphthalene-2,2dicarboxylate

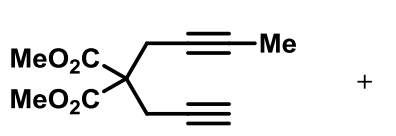

19<smiles>O=C1C=CC(=O)C=C1</smiles>

2a

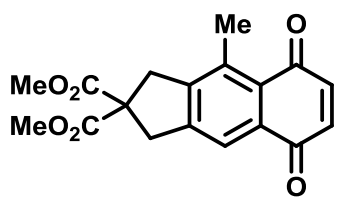

3ga

General Procedure C: Diyne 1g (22.2 mg, $0.100 \mathrm{mmol})$ and 1,4-benzoquinone (2a) (21.6 mg, $0.200 \mathrm{mmol})$ were employed. Purification by flash column chromatography (25\% EtOAc/Hex) on silica gel afforded the title compound (28.6 mg, 87\%) as a yellow solid; $\mathbf{R}_{f} 0.46$ (33\% EtOAc/Hex); mp 143$145^{\circ} \mathrm{C}\left(\mathrm{CH}_{2} \mathrm{Cl}_{2} / \mathrm{Et}_{2} \mathrm{O}\right) ; \mathbf{v}_{\max } / \mathrm{cm}^{-1}: 2958$ (w), 1731 (s), 1655 (s), 1592 (m), 1435 (m), 1328 (m), 1252 (s); ${ }^{1} \mathbf{H}$ NMR $\left(400 \mathrm{MHz}, \mathrm{CDCl}_{3}\right) \delta 7.84(1 \mathrm{H}, \mathrm{s}), 6.88-6.81(2 \mathrm{H}, \mathrm{m}), 3.77(6 \mathrm{H}, \mathrm{s}), 3.71(2 \mathrm{H}, \mathrm{s}), 3.67(2 \mathrm{H}$, s), $2.65(3 \mathrm{H}, \mathrm{s}) ;{ }^{13} \mathrm{C}$ NMR (101 MHz, $\left.\mathrm{CDCl}_{3}\right) \delta 187.4(\mathrm{C}), 185.6(\mathrm{C}), 171.6(2 \times \mathrm{C}), 147.3(\mathrm{C}), 145.5$ (C), $140.6(\mathrm{CH}), 137.5(\mathrm{C}), 136.8(\mathrm{CH}), 133.4(\mathrm{C}), 128.9(\mathrm{C}), 120.9(\mathrm{CH}), 59.4(\mathrm{C}), 53.4\left(2 \times \mathrm{CH}_{3}\right)$, $41.2\left(\mathrm{CH}_{2}\right), 40.4\left(\mathrm{CH}_{2}\right), 18.3\left(\mathrm{CH}_{3}\right)$; HRMS $\left(\mathrm{ESI}^{+}\right)$: Calculated for $\mathrm{C}_{18} \mathrm{H}_{17} \mathrm{O}_{6}: 329.1020$. Found [M + $\mathrm{H}]^{+}: 329.1025$. 


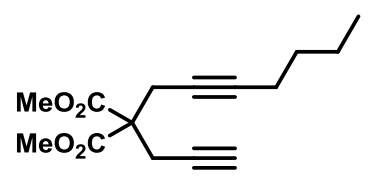

$1 \mathrm{~h}$

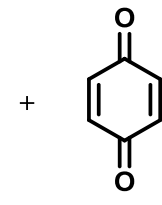

$2 \mathbf{a}$

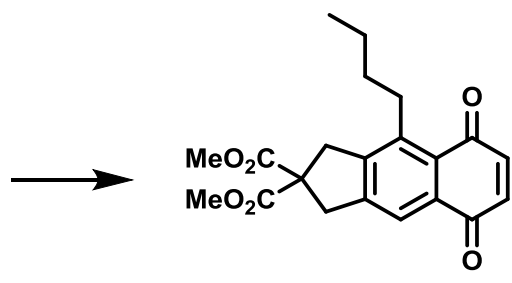

3ha

General Procedure C: Diyne $\mathbf{1 h}(26.4 \mathrm{mg}, 0.100 \mathrm{mmol})$ and 1,4-benzoquinone (2a) $(21.6 \mathrm{mg}$, $0.200 \mathrm{mmol})$ were employed. Purification by flash column chromatography (25\% EtOAc/Hex) on silica gel afforded the title compound $(24.7 \mathrm{mg}, 67 \%)$ as a yellow oil; $\mathbf{R}_{f} 0.56(33 \% \mathrm{EtOAc} / \mathrm{Hex}) ; \mathbf{v}_{\max } / \mathrm{cm}^{-1}$ : 2957 (w), 1733 (s), 1656 (s), 1614 (m), 1592 (m), 1435 (m), 1251 (s); ${ }^{1} \mathbf{H}$ NMR (400 MHz, CDCl $\left.{ }_{3}\right) \delta$ $7.85(1 \mathrm{H}, \mathrm{s}), 6.88-6.79(2 \mathrm{H}, \mathrm{m}), 3.77(6 \mathrm{H}, \mathrm{s}), 3.70(2 \mathrm{H}, \mathrm{s}), 3.67(2 \mathrm{H}, \mathrm{s}), 3.08(2 \mathrm{H}, \mathrm{m}), 1.57-1.45(4 \mathrm{H}$, m), $0.98(3 \mathrm{H}, \mathrm{t}, J=7.0 \mathrm{~Hz}) ;{ }^{13} \mathbf{C ~ N M R}\left(101 \mathrm{MHz}, \mathrm{CDCl}_{3}\right) \delta 187.1(\mathrm{C}), 185.7(\mathrm{C}), 171.6(2 \times \mathrm{C}), 147.1$ (C), $145.8(\mathrm{C}), 142.5(\mathrm{C}), 140.8(\mathrm{CH}), 136.6(\mathrm{CH}), 133.7(\mathrm{C}), 128.3(\mathrm{C}), 121.2(\mathrm{CH}), 59.6(\mathrm{C}), 53.4(2$ $\left.\times \mathrm{CH}_{3}\right), 41.1\left(\mathrm{CH}_{2}\right), 39.8\left(\mathrm{CH}_{2}\right), 31.7\left(\mathrm{CH}_{2}\right), 31.1\left(\mathrm{CH}_{2}\right), 23.5\left(\mathrm{CH}_{2}\right), 14.1\left(\mathrm{CH}_{3}\right) ; \mathbf{H R M S}\left(\mathrm{ESI}^{+}\right)$: Calculated for $\mathrm{C}_{21} \mathrm{H}_{23} \mathrm{O}_{6}: 371.1489$. Found $[\mathrm{M}+\mathrm{H}]^{+}: 371.1501$.

(3ia): Dimethyl 4-((tert-butyldimethylsilyl)oxymethyl)-5,8-dioxo-1,3,5,8-tetrahydro-2Hcyclopenta[b]naphthalene-2,2-dicarboxylate

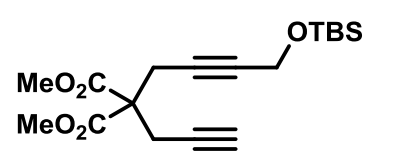

$1 \mathbf{i}$

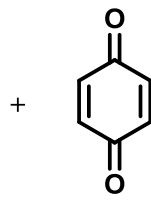

2a

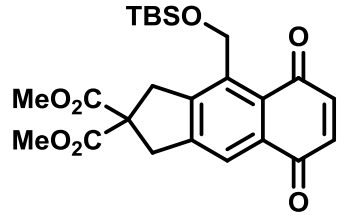

3ia

General Procedure C: Diyne 1i $(35.2 \mathrm{mg}, 0.100 \mathrm{mmol})$ and 1,4-benzoquinone (2a) $(21.6 \mathrm{mg}$, $0.200 \mathrm{mmol})$ were employed. Purification by flash column chromatography ( $20 \%$ EtOAc/Hex) on silica gel afforded the title compound $(37.5 \mathrm{mg}, 82 \%)$ as a green oil which solidified on standing; $\mathbf{R}_{f} 0.66$ (33\% EtOAc/Hex); $\boldsymbol{v}_{\max } / \mathrm{cm}^{-1}: 2954$ (w), 2857 (w), 1735 (s), 1660 (s), 1593 (m), 1434 (m), 1253 (s); ${ }^{1} \mathbf{H}$ NMR $\left(400 \mathrm{MHz}, \mathrm{CDCl}_{3}\right) \delta 7.93(1 \mathrm{H}, \mathrm{s}), 6.86(2 \mathrm{H}, \mathrm{m}), 5.28(2 \mathrm{H}, \mathrm{s}), 3.89(2 \mathrm{H}, \mathrm{s}), 3.76(6 \mathrm{H}, \mathrm{s}), 3.67$ $(2 \mathrm{H}, \mathrm{s}), 0.92(9 \mathrm{H}, \mathrm{s}), 0.13(6 \mathrm{H}, \mathrm{s}) ;{ }^{13} \mathbf{C} \mathbf{N M R}\left(101 \mathrm{MHz}, \mathrm{CDCl}_{3}\right) \delta 187.3(\mathrm{C}), 185.3(\mathrm{C}), 171.7(2 \times \mathrm{C})$, $147.9(\mathrm{C}), 146.9(\mathrm{C}), 140.6(\mathrm{CH}), 139.6(\mathrm{C}), 136.7(\mathrm{CH}), 133.1(\mathrm{C}), 128.5(\mathrm{C}), 122.4(\mathrm{CH}), 60.9\left(\mathrm{CH}_{2}\right)$, $59.8(\mathrm{C}), 53.3\left(2 \times \mathrm{CH}_{3}\right), 41.3\left(\mathrm{CH}_{2}\right), 40.6\left(\mathrm{CH}_{2}\right), 26.1\left(3 \times \mathrm{CH}_{3}\right), 18.6(\mathrm{C}),-5.2\left(2 \times \mathrm{CH}_{3}\right) ; \mathbf{H R M S}$ $\left(\mathrm{ESI}^{+}\right)$: Calculated for $\mathrm{C}_{24} \mathrm{H}_{31} \mathrm{O}_{7} \mathrm{Si}: 459.1834$. Found $[\mathrm{M}+\mathrm{H}]^{+}: 459.1832$. 
(3ja): Dimethyl 5,8-dioxo-4,9-diphenyl-1,3,5,8-tetrahydro-2H-cyclopenta[b]naphthalene-2,2dicarboxylate

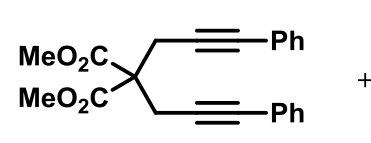

$1 \mathbf{j}$<smiles>O=C1C=CC(=O)C=C1</smiles>

2a

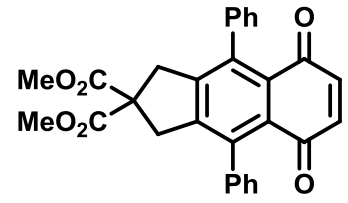

$3 \mathbf{j a}$

General Procedure C: Diyne 1j (104 mg, $0.288 \mathrm{mmol}$ ) and 1,4-benzoquinone (2a) (62.3 mg, $0.576 \mathrm{mmol}$ ) were employed. Purification by flash column chromatography ( $5 \%$ acetone/PhMe) on silica gel afforded the title compound $(69.8 \mathrm{mg}, 52 \%)$ as a yellow oil that solidified on standing; $\mathbf{R}_{f} 0.32$ (33\% EtOAc/Hex); mp $188-190{ }^{\circ} \mathrm{C}\left(\mathrm{CH}_{2} \mathrm{Cl}_{2} / \mathrm{Et}_{2} \mathrm{O}\right) ; \mathbf{v}_{\max } / \mathrm{cm}^{-1}$ : 2954 (w), 1734 (s), 1664 (s), 1435 (w), 1255 (s), 1073 (m); ${ }^{1} \mathbf{H}$ NMR (400 MHz, $\left.\mathrm{CDCl}_{3}\right) \delta$ 7.51-7.40 (6H, m), 7.21-7.16 (4H, m), $6.68(2 \mathrm{H}$, s), $3.68(6 \mathrm{H}, \mathrm{s}), 3.36(4 \mathrm{H}, \mathrm{s}) ;{ }^{13} \mathbf{C} \mathbf{~ N M R}\left(101 \mathrm{MHz}, \mathrm{CDCl}_{3}\right) \delta 185.9(2 \times \mathrm{C}), 171.5(2 \times \mathrm{C}), 146.5(2 \times$ C), $139.7(2 \times \mathrm{C}), 139.3(2 \times \mathrm{C}), 138.5(2 \times \mathrm{CH}), 130.2(2 \times \mathrm{C}), 128.8(4 \times \mathrm{CH}), 127.3(6 \times \mathrm{CH}), 59.0$ (C), $53.3\left(2 \times \mathrm{CH}_{3}\right), 41.1\left(2 \times \mathrm{CH}_{2}\right)$; HRMS $\left(\mathrm{ESI}^{+}\right)$: Calculated for $\mathrm{C}_{29} \mathrm{H}_{23} \mathrm{O}_{6}: 467.1489$. Found [M + $\mathrm{H}]^{+}: 467.1493$.

(3ka): Dimethyl 4,9-bis(4-methoxyphenyl)-5,8-dioxo-1,3,5,8-tetrahydro-2Hcyclopenta[$[b]$ naphthalene-2,2-dicarboxylate

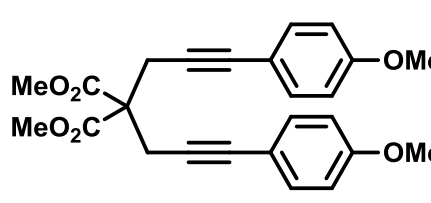

$1 \mathrm{k}$<smiles>O=C1C=CC(=O)C=C1</smiles>

$\mathbf{2 a}$

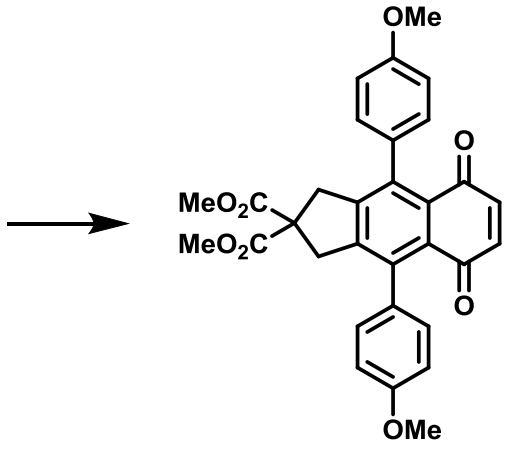

$3 \mathrm{ka}$

General Procedure C: Diyne 1k (42.1 mg, $0.100 \mathrm{mmol})$ and 1,4-benzoquinone (2a) $(21.6 \mathrm{mg}$, $0.200 \mathrm{mmol})$ were employed. Purification by flash column chromatography ( $10 \%$ acetone/ $\mathrm{PhMe})$ on silica gel afforded the title compound (30.5 mg, 58\%) as an orange oil; $\mathbf{R}_{f} 0.68$ (10\% acetone/PhMe); $\boldsymbol{v}_{\max } / \mathrm{cm}^{-1}: 2954$ (w), 1734 (s), 1664 (s), 1516 (s), 1285 (m), 1245 (s); ${ }^{1} \mathbf{H}$ NMR (400 MHz, CDCl 3 ) $\delta$ 7.12-7.08 (4H, m), 7.03-6.99 (4H, m), 6.67 (2H, s), 3.87 (6H, s), 3.67 (6H, s), 3.38 (4H, s); ${ }^{13}$ C NMR $\left(101 \mathrm{MHz}, \mathrm{CDCl}_{3}\right) \delta 186.1(2 \times \mathrm{C}), 171.5(2 \times \mathrm{C}), 158.8(2 \times \mathrm{C}), 146.7(2 \times \mathrm{C}), 138.9(2 \times \mathrm{C}), 138.4$ $(2 \times \mathrm{CH}), 131.7(2 \times \mathrm{C}), 130.4(2 \times \mathrm{C}), 128.6(4 \times \mathrm{CH}), 114.2(4 \times \mathrm{CH}), 59.0(\mathrm{C}), 55.3\left(2 \times \mathrm{CH}_{3}\right), 53.2$ 
$\left(2 \times \mathrm{CH}_{3}\right), 41.2\left(2 \times \mathrm{CH}_{2}\right)$; HRMS $\left(\mathrm{ESI}^{+}\right)$: Calculated for $\mathrm{C}_{31} \mathrm{H}_{27} \mathrm{O}_{8}: 527.1700$. Found $[\mathrm{M}+\mathrm{H}]^{+}$: 527.1704 .

(3la): Dimethyl 4,9-bis(4-fluorophenyl)-5,8-dioxo-1,3,5,8-tetrahydro-2Hcyclopenta[b]naphthalene-2,2-dicarboxylate

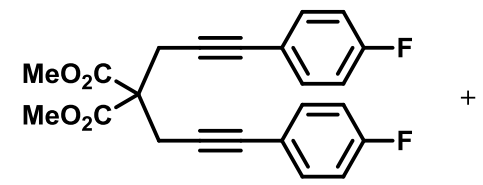

11<smiles>O=C1C=CC(=O)C=C1</smiles>

2a

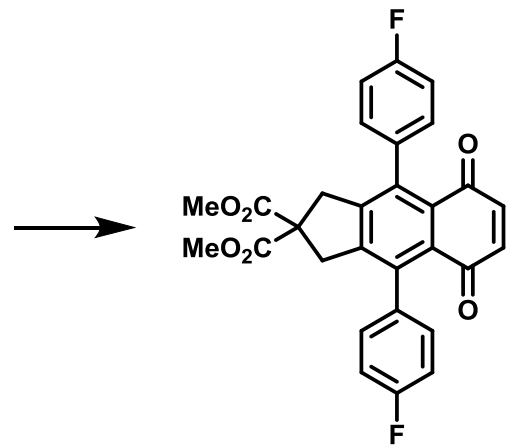

3la

General Procedure C: Diyne $11(39.6 \mathrm{mg}, 0.100 \mathrm{mmol})$ and 1,4-benzoquinone (2a) $(21.6 \mathrm{mg}$, $0.200 \mathrm{mmol}$ ) were employed. Purification by flash column chromatography (5\% acetone/PhMe) on silica gel afforded the title compound $(15.7 \mathrm{mg}, 30 \%)$ as a yellow solid; $\mathbf{R}_{f} 0.55$ (33\% EtOAc/Hex); $\mathbf{m p}$ 181-183 ${ }^{\circ} \mathrm{C}\left(\mathrm{CH}_{2} \mathrm{Cl}_{2} / \mathrm{Et}_{2} \mathrm{O}\right) ; \mathbf{v}_{\max } / \mathrm{cm}^{-1}: 2955$ (w), 1734 (s), 1665 (s), 1514 (s), 1436 (m), 1331 (m), 1255 (s); ${ }^{1} \mathrm{H}$ NMR (400 MHz, $\left.\mathrm{CDCl}_{3}\right) \delta$ 7.21-7.11 (8H, m), $6.70(2 \mathrm{H}, \mathrm{s}), 3.69$ (6H, s), 3.34 (4H, s); ${ }^{13} \mathrm{C} \mathrm{NMR}\left(101 \mathrm{MHz}, \mathrm{CDCl}_{3}\right) \delta 185.8(2 \times \mathrm{C}), 171.4(2 \times \mathrm{C}), 162.2\left(2 \times \mathrm{C}, \mathrm{d},{ }^{1} J_{\mathrm{CF}}=246.0 \mathrm{~Hz}\right), 146.7$ $(2 \times \mathrm{C}), 138.51(2 \times \mathrm{CH}), 138.47(2 \times \mathrm{C}), 135.3\left(2 \times \mathrm{C}, \mathrm{d},{ }^{4} J_{\mathrm{CF}}=3.5 \mathrm{~Hz}\right), 130.4(2 \times \mathrm{C}), 129.0(4 \times \mathrm{CH}$, $\left.\mathrm{d},{ }^{3} J_{\mathrm{CF}}=8.0 \mathrm{~Hz}\right), 115.9\left(4 \times \mathrm{CH}, \mathrm{d},{ }^{2} J_{\mathrm{CF}}=21.5 \mathrm{~Hz}\right), 59.0(\mathrm{C}), 53.3\left(2 \times \mathrm{CH}_{3}\right), 41.1\left(2 \times \mathrm{CH}_{2}\right) ; \mathbf{F}^{\mathbf{1 9}} \mathbf{N M R}$ $\left(376 \mathrm{MHz}, \mathrm{CDCl}_{3}\right) \delta-114.8$; HRMS $\left(\mathrm{ESI}^{+}\right)$: Calculated for $\mathrm{C}_{29} \mathrm{H}_{21} \mathrm{~F}_{2} \mathrm{O}_{6}: 503.1301$. Found $[\mathrm{M}+\mathrm{H}]^{+}$: 503.1309.

(3ma): 11-Phenyl-5-tosyl-5H-benzo[b]carbazole-7,10-dione<smiles>C#C[N+](C)c1ccccc1C#Cc1ccccc1</smiles>

$1 \mathrm{~m}$<smiles>O=C1C=CC(=O)C=C1</smiles>

2a<smiles>Cn1c2ccccc2c2c(-c3ccccc3)c3c(cc21)C(=O)C=CC3=O</smiles>

$3 \mathrm{ma}$

General Procedure B: Diyne $\mathbf{1 m}(37.1 \mathrm{mg}, 0.100 \mathrm{mmol})$ and 1,4-benzoquinone (2a) $(21.6 \mathrm{mg}$, $0.200 \mathrm{mmol})$ were employed. Purification by flash column chromatography (25\%-50\% EtOAc/Hex) on silica gel afforded the title compound (16.8 mg, 35\%) as green solid; $\mathbf{R}_{f} 0.38$ (33\% EtOAc/Hex); $\mathbf{m p}$ 233-235 ${ }^{\circ} \mathrm{C}\left(\mathrm{CH}_{2} \mathrm{Cl}_{2} / \mathrm{Et}_{2} \mathrm{O}\right) ; \mathbf{v}_{\max } / \mathrm{cm}^{-1}: 3060$ (w), 2922 (w), 1664 (s), 1613 (m), 1574 (m), 1376 (m), 
1326 (s) 1177 (s); ${ }^{1} \mathbf{H}$ NMR (400 MHz, CDCl $) \delta 9.15(1 \mathrm{H}, \mathrm{s}), 8.38(1 \mathrm{H}, \mathrm{d}, J=8.5 \mathrm{~Hz}), 7.85(2 \mathrm{H}, \mathrm{d}, J$ $=8.5 \mathrm{~Hz}), 7.59-7.53(3 \mathrm{H}, \mathrm{m}), 7.48(1 \mathrm{H}, \mathrm{ddd}, J=8.5,7.5,1.5 \mathrm{~Hz}), 7.25-7.18(4 \mathrm{H}, \mathrm{m}), 7.06-6.98(2 \mathrm{H}$, m), $6.79(1 \mathrm{H}, \mathrm{d}, J=10.0 \mathrm{~Hz}), 6.33-6.28(1 \mathrm{H}, \mathrm{m}), 2.32(3 \mathrm{H}, \mathrm{s}) ;{ }^{13} \mathbf{C ~ N M R}\left(101 \mathrm{MHz}, \mathrm{CDCl}_{3}\right) \delta 185.1$ (C), $185.0(\mathrm{C}), 145.9(\mathrm{C}), 140.8(\mathrm{CH}), 140.1(\mathrm{C}), 140.0(\mathrm{C}), 139.2(\mathrm{C}), 138.9(\mathrm{C}), 137.5(\mathrm{CH}), 134.9$ $(\mathrm{C}), 131.8(\mathrm{C}), 130.2(2 \times \mathrm{CH}), 129.6(\mathrm{C}), 129.3(2 \times \mathrm{CH}), 129.2(\mathrm{CH}), 128.0(\mathrm{CH}), 127.5(2 \times \mathrm{CH})$, $126.9(2 \times \mathrm{CH}), 125.3(\mathrm{C}), 124.9(\mathrm{C}), 124.3(\mathrm{CH}), 123.8(\mathrm{CH}), 114.6(\mathrm{CH}), 113.1(\mathrm{CH}), 21.7\left(\mathrm{CH}_{3}\right)$; HRMS (ESI $\left.{ }^{+}\right)$: Calculated for $\mathrm{C}_{29} \mathrm{H}_{20} \mathrm{NO}_{4} \mathrm{~S}: 478.1108$. Found $[\mathrm{M}+\mathrm{H}]^{+}: 478.1103$.

(3na): 11-Phenylnaphtho[2,3-b]benzofuran-7,10-dione

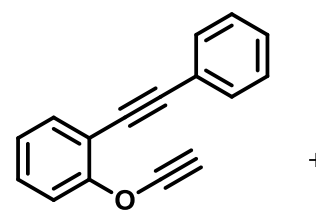

$1 \mathrm{n}$<smiles>O=C1C=CC(=O)C=C1</smiles>

$2 \mathbf{a}$

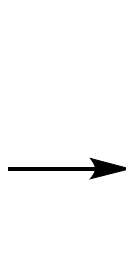<smiles>O=C1C=CC(=O)c2c1cc1oc3ccccc3c1c2-c1ccccc1</smiles>

3na

General Procedure B: Diyne 1n (21.8 mg, $0.100 \mathrm{mmol})$ and 1,4-benzoquinone (2a) (21.6 mg, $0.200 \mathrm{mmol})$ were employed. Purification by flash column chromatography ( $1 \%$ acetone/PhMe) on silica gel afforded the title compound ( $24.8 \mathrm{mg}, 76 \%)$ as a yellow solid; $\mathbf{R}_{f} 0.58$ (2\% acetone/ $\mathrm{PhMe}$ ); mp 275-276 ${ }^{\circ} \mathrm{C}\left(\mathrm{CH}_{2} \mathrm{Cl}_{2} / \mathrm{Et}_{2} \mathrm{O} ; \mathbf{v}_{\max } / \mathrm{cm}^{-1}: 2916\right.$ (m), 2851 (w), 1662 (s), 1612 (m), 1335 (s), 1279 (s), 1075 (s); ${ }^{1} \mathrm{H}$ NMR $\left(400 \mathrm{MHz}, \mathrm{CDCl}_{3}\right) \delta 8.35(1 \mathrm{H}, \mathrm{s}), 7.62-7.56(4 \mathrm{H}, \mathrm{m}), 7.47(1 \mathrm{H}$, ddd, $J=8.5,7.5$, $1.5 \mathrm{~Hz}), 7.35-7.30(2 \mathrm{H}, \mathrm{m}), 7.08(1 \mathrm{H}, \mathrm{ddd}, J=8.0,7.5,1.0 \mathrm{~Hz}), 7.00(1 \mathrm{H}, \mathrm{d}, J=10.0 \mathrm{~Hz}), 6.82(1 \mathrm{H}, \mathrm{d}$, $J=10.0 \mathrm{~Hz}), 6.53(1 \mathrm{H}, \mathrm{ddd}, J=8.0,1.0,0.5 \mathrm{~Hz}) ;{ }^{13} \mathbf{C} \mathbf{N M R}\left(101 \mathrm{MHz}, \mathrm{CDCl}_{3}\right) \delta 185.1(\mathrm{C}), 185.0(\mathrm{C})$, $158.0(\mathrm{C}), 157.5(\mathrm{C}), 140.8(\mathrm{CH}), 139.8(\mathrm{C}), 138.8(\mathrm{C}), 137.5(\mathrm{CH}), 132.7(\mathrm{C}), 129.3(\mathrm{CH}), 129.2(2 \times$ $\mathrm{CH}+\mathrm{C}), 128.0(\mathrm{CH}), 127.4(2 \times \mathrm{CH}), 124.7(\mathrm{C}), 123.8(\mathrm{CH}), 123.6(\mathrm{CH}), 123.4(\mathrm{C}), 112.1(\mathrm{CH}), 110.2$ $(\mathrm{CH})$; HRMS $\left(\mathrm{ESI}^{+}\right)$: Calculated for $\mathrm{C}_{22} \mathrm{H}_{13} \mathrm{O}_{3}: 325.0859$. Found $[\mathrm{M}+\mathrm{H}]^{+}: 325.0859$. 


\section{Experimental Observations and Derivatizations}

(3ea'): Dimethyl (4a $\left.R^{*}, 8 \mathrm{a} S^{*}\right)-4,9-d i m e t h y l-5,8-d i o x o-1,3,4 a, 5,8,8 \mathrm{a}-h e x a h y d r o-2 H$ cyclopenta[b]naphthalene-2,2-dicarboxylate

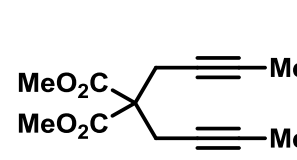

1e<smiles>O=C1C=CC(=O)C=C1</smiles>

$2 a$

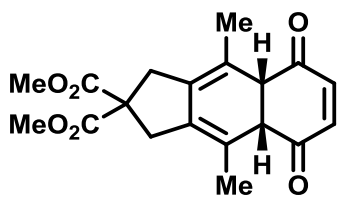

$3 e a^{\prime}$

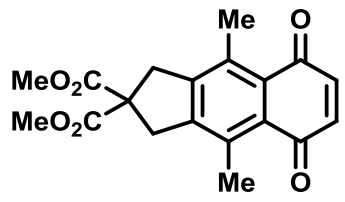

3ea

General Procedure B: Diyne 1e $(68.1 \mathrm{mg}, 0.2882 \mathrm{mmol})$ and 1,4-benzoquinone (2a) $(62.3 \mathrm{mg}$, $0.576 \mathrm{mmol}$ ) were employed. Purification by flash column chromatography (5\% acetone $/ \mathrm{PhMe}$ ) on silica gel afforded the title compound $(42.5 \mathrm{mg}, 43 \%)$ as an orange-yellow solid, along with 3ea (36.6 mg, 37\%) as a yellow solid; $\mathbf{R}_{f} 0.33$ (33\% EtOAc/Hex); $\mathbf{m p} 120-122{ }^{\circ} \mathrm{C}\left(\mathrm{CH}_{2} \mathrm{Cl}_{2} / \mathrm{Et}_{2} \mathrm{O}\right)$; $\mathbf{v}_{\max } /$ cm ${ }^{-1}$ : 2924 (w), 1733 (s), 1684 (s), 1435 (m), 1256 (s); ${ }^{1} \mathbf{H}$ NMR (400 MHz, CDCl 3 ) $\delta 6.65$ (2H, s), $3.71(3 \mathrm{H}, \mathrm{s}), 3.70(3 \mathrm{H}, \mathrm{s}), 3.46(2 \mathrm{H}, \mathrm{s}), 2.96(4 \mathrm{H}, \mathrm{s}), 1.73(6 \mathrm{H}, \mathrm{s}) ;{ }^{13} \mathbf{C} \mathbf{N M R}\left(101 \mathrm{MHz}, \mathrm{CDCl}_{3}\right) \delta 200.4$ $(2 \times \mathrm{C}), 171.9(\mathrm{C}), 171.7(\mathrm{C}), 140.3(2 \times \mathrm{CH}), 134.0(2 \times \mathrm{C}), 120.9(2 \times \mathrm{C}), 58.8(\mathrm{C}), 54.1\left(2 \times \mathrm{CH}_{3}\right)$, $53.1(2 \times \mathrm{CH}), 37.2\left(2 \times \mathrm{CH}_{2}\right), 18.5\left(2 \times \mathrm{CH}_{3}\right)$; HRMS $\left(\mathrm{ESI}^{+}\right)$: Calculated for $\mathrm{C}_{19} \mathrm{H}_{21} \mathrm{O}_{6}: 345.1333$. Found $[\mathrm{M}+\mathrm{H}]^{+}: 345.1329$.

(3ea): Dimethyl 4,9-dimethyl-5,8-dioxo-1,3,5,8-tetrahydro-2H-cyclopenta[b]naphthalene-2,2dicarboxylate

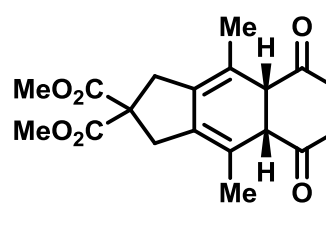

3 ea'

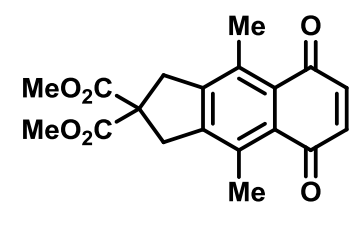

3ea

A flame-dried Schlenk tube, fitted with a magnetic stirrer, was charged with enone $\mathbf{3 e a}$ ' $10.0 \mathrm{mg}$, $29.0 \mu \mathrm{mol})$ and 1,4-benzoquinone (2a) $(15.7 \mathrm{mg}, 145 \mu \mathrm{mol})$. The Schlenk tube was fitted with a rubber septum, evacuated and refilled with nitrogen (three cycles), then DCE $(0.29 \mathrm{~mL})$ was added via syringe. The tube was sealed with a PTFE cap and placed in a pre-heated heating block at $100{ }^{\circ} \mathrm{C}$ for 18 hours. The reaction mixture was cooled to room temperature and concentrated in vacuo. The crude reaction mixture was purified by flash column chromatography ( $5 \%$ acetone/PhMe) on silica gel afforded the title compound $(6.7 \mathrm{mg}, 67 \%)$ as a yellow solid. 
(5): 2,2-Bis(methoxycarbonyl)-5,8-dioxo-2,3,5,8-tetrahydro- $1 H$-cyclopenta[b]naphthalene-4carboxylic acid

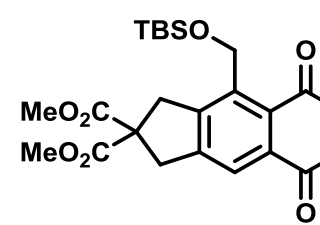

3 ia

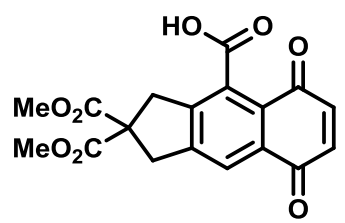

5

To a solution of silyl ether 3ia $(60.0 \mathrm{mg}, 131 \mu \mathrm{mol})$ in acetone $(3 \mathrm{~mL})$ at $0{ }^{\circ} \mathrm{C}$ was added Jones reagent ( $0.3 \mathrm{~mL}$, prepared from $250 \mathrm{mg}$ of $\mathrm{CrO}_{3}$ and $250 \mu \mathrm{L}$ of $\mathrm{H}_{2} \mathrm{SO}_{4}$ diluted to $1 \mathrm{~mL}$ with $\mathrm{H}_{2} \mathrm{O}$ ). The reaction mixture was stirred at this temperature for $1 \mathrm{~h}$, then poured into cold water $(5 \mathrm{~mL})$. The aqueous layer was extracted with $\mathrm{CH}_{2} \mathrm{Cl}_{2}(3 \times 5 \mathrm{~mL})$, and the combined organic layers were dried over anhydrous $\mathrm{Na}_{2} \mathrm{SO}_{4}$ and concentrated in vacuo. Purification by flash column chromatography (10\% $\mathrm{MeOH} / \mathrm{CH}_{2} \mathrm{Cl}_{2}$ with $0.5 \% \mathrm{AcOH})$ on silica gel afforded the title compound $(27.2 \mathrm{mg}, 58 \%)$ as a yellow solid; $\mathbf{R}_{f} 0.32$ (10\% MeOH/CH $\mathrm{CH}_{2} \mathrm{Cl}_{2}$ with 0.5\% AcOH); mp $183.3-184.5^{\circ} \mathrm{C}\left(\mathrm{CH}_{2} \mathrm{Cl}_{2} / \mathrm{Et}_{2} \mathrm{O}\right) ; v_{\max } / \mathrm{cm}^{-1}: 2956$ (w), 1721 (s), 1664 (s), 1594 (m), 1432 (m), 1290 (s); ${ }^{1} \mathbf{H}$ NMR (400 MHz, CDCl $)$ ) 7.99 (1H, s), 6.96 (2H, m), 3.77 (6H, s), 3.76 (2H, s), 3.74 (2H, s); $\left.{ }^{13} \mathbf{C ~ N M R ~ ( 1 0 1 ~ M H z , ~} \mathrm{CDCl}_{3}\right) \delta 184.3$ (C), $184.2(\mathrm{C}), 172.6$ $(\mathrm{C}), 171.2(2 \times \mathrm{C}), 147.6(\mathrm{C}), 144.2(\mathrm{C}), 138.8(\mathrm{CH}), 138.4(\mathrm{CH}), 131.9(\mathrm{C}), 129.8(\mathrm{C}), 128.2(\mathrm{C}), 123.5$ $(\mathrm{CH}), 59.9(\mathrm{C}), 53.5\left(2 \times \mathrm{CH}_{2}\right), 40.8\left(\mathrm{CH}_{2}\right), 39.1\left(\mathrm{CH}_{2}\right)$; HRMS (TOF): Calculated for $\mathrm{C}_{18} \mathrm{H}_{13} \mathrm{O}_{8}$ : 357.0610. Found $[\mathrm{M}-\mathrm{H}]^{-}: 357.0605$.

1 mmol Scale Synthesis of 3ac:

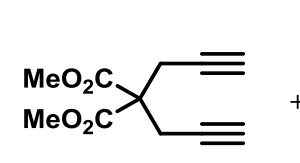

$1 a$<smiles>COC1=CC(=O)C=CC1=O</smiles>

2c

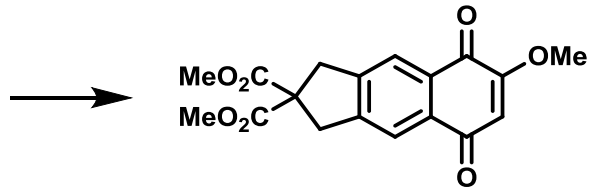

3ac

General Procedure B: Diyne 1a (208 mg, $1.00 \mathrm{mmol})$ and benzoquinone 2c (276 mg, $2.00 \mathrm{mmol})$, $\left[\mathrm{Rh}(\mathrm{coe}) \mathrm{Cl}_{2}\right]_{2}(17.9 \mathrm{mg}, 0.025 \mathrm{mmol}, 2.5 \mathrm{~mol} \%)$ and $\left(4-\mathrm{NCC}_{6} \mathrm{H}_{4}\right)_{3} \mathrm{P}(33.7 \mathrm{mg}, 0.100 \mathrm{mmol}, 10 \mathrm{~mol} \%)$ were employed. Purification by flash column chromatography (10\% acetone/PhMe) on silica gel afforded the title compound ( $255 \mathrm{mg}, 74 \%)$ as a pale yellow solid. 


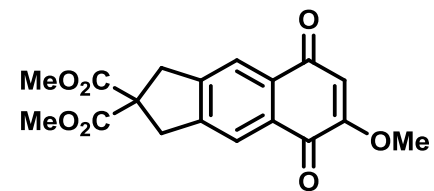

3ac

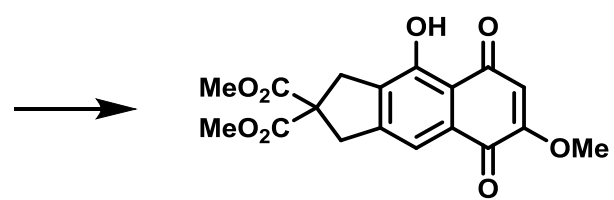

6

A flame-dried Schlenk tube was charged with naphthoquinone 3ac $(50.0 \mathrm{mg}, 0.145 \mathrm{mmol})$, [bis(trifluoroacetoxy)iodo]benzene $(74.9 \mathrm{mg}, \quad 0.174 \mathrm{mmol})$ and $\left[\mathrm{RuCl}_{2}(p \text {-cymene })\right]_{2} \quad(2.22 \mathrm{mg}$, $3.63 \mu \mathrm{L})$. Trifluoroacetic anhydride $(0.36 \mathrm{~mL})$ and trifluoroacetic acid $(7 \mu \mathrm{L})$ were subsequently added. The reaction tube was sealed and the mixture stirred at $80^{\circ} \mathrm{C}$ for $16 \mathrm{~h}$, then cooled to room temperature. The mixture was transferred dropwise to a flask containing $\mathrm{CH}_{2} \mathrm{Cl}_{2}(0.5 \mathrm{~mL}), \mathrm{H}_{2} \mathrm{O}(0.5 \mathrm{~mL})$ and $1 \mathrm{M}$ aq $\mathrm{HCl}(0.1 \mathrm{~mL})$ under vigorous stirring. After $5 \mathrm{~min}$, the biphasic mixture was extracted with $\mathrm{CH}_{2} \mathrm{Cl}_{2}$ $(3 \times 5 \mathrm{~mL})$ and the combined organic extracts were dried over anhydrous $\mathrm{Na}_{2} \mathrm{SO}_{4}$ and concentrated in vacuo. Purification by flash column chromatography (50\% EtOAc/Hex) on silica gel afforded the title compound (42.3 mg, 81\%) as an orange-yellow solid; $\mathbf{R}_{f} 0.65$ (66\% EtOAc/Hex); $\mathbf{m p ~} 157-159{ }^{\circ} \mathrm{C}$ $\left(\mathrm{CH}_{2} \mathrm{Cl}_{2} / \mathrm{Et}_{2} \mathrm{O}\right) ; v_{\max } / \mathrm{cm}^{-1}: 2955$ (w), 1733 (s), 1683 (m), 1633 (s), 1600 (s), 1432 (s), 1280 (s), 1245 (s); ${ }^{1} \mathbf{H}$ NMR $\left(400 \mathrm{MHz}, \mathrm{CDCl}_{3}\right) \delta 12.32(1 \mathrm{H}, \mathrm{s}), 7.52(1 \mathrm{H}, \mathrm{s}), 6.03(1 \mathrm{H}, \mathrm{s}), 3.90(3 \mathrm{H}, \mathrm{s}), 3.77(6 \mathrm{H}, \mathrm{s})$, $3.65(4 \mathrm{H}, \mathrm{s}) ;{ }^{13} \mathrm{C}$ NMR $\left(100 \mathrm{MHz}, \mathrm{CDCl}_{3}\right) \delta 190.9(\mathrm{C}), 179.5(\mathrm{C}), 171.5(2 \times \mathrm{C}), 161.2(\mathrm{C}), 157.5(\mathrm{C})$, $148.9(\mathrm{C}), 135.9(\mathrm{C}), 131.1(\mathrm{C}), 115.9(\mathrm{CH}), 113.4(\mathrm{C}), 109.4(\mathrm{CH}), 59.7(\mathrm{C}), 56.8\left(\mathrm{CH}_{3}\right), 53.4(2 \times$ $\left.\mathrm{CH}_{3}\right), 41.4\left(\mathrm{CH}_{2}\right), 37.6\left(\mathrm{CH}_{2}\right)$; HRMS $\left(\mathrm{ESI}^{+}\right)$: Calculated for $\mathrm{C}_{18} \mathrm{H}_{17} \mathrm{O}_{8}: 361.0918$. Found $[\mathrm{M}+\mathrm{H}]^{+}$: 361.0931 .

(7): Dimethyl 4,9-dihydroxy-6-methoxy-5,8-dioxo-1,3,5,8-tetrahydro-2Hcyclopenta $[b]$ naphthalene-2,2-dicarboxylate<smiles>COC(=O)C1(C(=O)OC)Cc2cc3c(c(O)c2C1)C(=O)C=C(OC)C3=O</smiles>

6

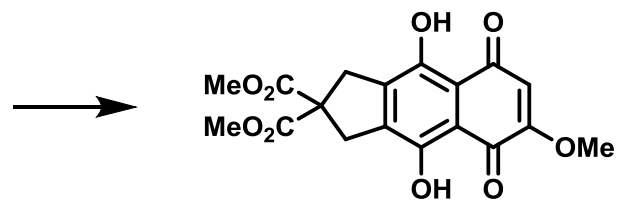

7

A flame-dried Schlenk tube was charged with naphthoquinone $6(20.0 \mathrm{mg}, 55.5 \mu \mathrm{mol})$ and [bis(trifluoroacetoxy)iodo]benzene $(47.7 \mathrm{mg}, 111 \mu \mathrm{mol})$ then placed under an atmosphere of $\mathrm{N}_{2}$. $\mathrm{CH}_{2} \mathrm{Cl}_{2}(0.17 \mathrm{~mL})$ was added via syringe and the reaction tube was sealed, then stirred at $80{ }^{\circ} \mathrm{C}$ for $16 \mathrm{~h}$. The yellow-orange reaction mixture was cooled to room temperature, then concentrated in vacuo with silica gel (approx. $150 \mathrm{mg}$ ) upon which it turned red. The resultant solid was purified by flash column chromatography (50\% EtOAc/Hex) on silica gel to afford the title compound (15.6 mg, 75\%) as a red 
solid; $\mathbf{R}_{f} 0.48$ (50\% EtOAc/Hex); mp 124-126 ${ }^{\circ} \mathrm{C}\left(\mathrm{CH}_{2} \mathrm{Cl}_{2} / \mathrm{Et}_{2} \mathrm{O}\right) ; \mathbf{v}_{\max } / \mathrm{cm}^{-1}: 2958(\mathrm{~m}), 2924(\mathrm{~m})$, 1733 (s), 1597 (s), 1429 (s), 1242 (s), 1080 (s); ${ }^{1} \mathbf{H}$ NMR (400 MHz, CDCl $) \delta 12.78(1 \mathrm{H}, \mathrm{s}), 12.33(1 \mathrm{H}$, s), $6.12(1 \mathrm{H}, \mathrm{s}), 3.92(3 \mathrm{H}, \mathrm{s}), 3.79(6 \mathrm{H}, \mathrm{s}), 3.69(2 \mathrm{H}, \mathrm{s}), 3.68(2 \mathrm{H}, \mathrm{s}) ;{ }^{13} \mathrm{C} \mathrm{NMR}\left(100 \mathrm{MHz}, \mathrm{CDCl}_{3}\right) \delta$ 187.9 (C), 181.9 (C), $171.4(2 \times$ C), 161.1 (C), 156.1 (C), 154.7 (C), 142.5 (C), 139.5 (C), 111.6 (C), $110.6(\mathrm{C}), 110.1(\mathrm{CH}), 59.2(\mathrm{C}), 56.9\left(\mathrm{CH}_{3}\right), 53.5\left(2 \times \mathrm{CH}_{3}\right), 38.6\left(\mathrm{CH}_{2}\right), 38.3\left(\mathrm{CH}_{2}\right) ; \mathbf{H R M S}\left(\mathrm{ESI}^{+}\right)$: Calculated for $\mathrm{C}_{18} \mathrm{H}_{17} \mathrm{O}_{9}: 377.0867$. Found $[\mathrm{M}+\mathrm{H}]^{+}: 377.0875$. 


\section{Total Synthesis of Justicidone}

(10): 3-(Benzo[d] $[1,3]$ dioxol-5-yl)prop-2-yn-1-ol

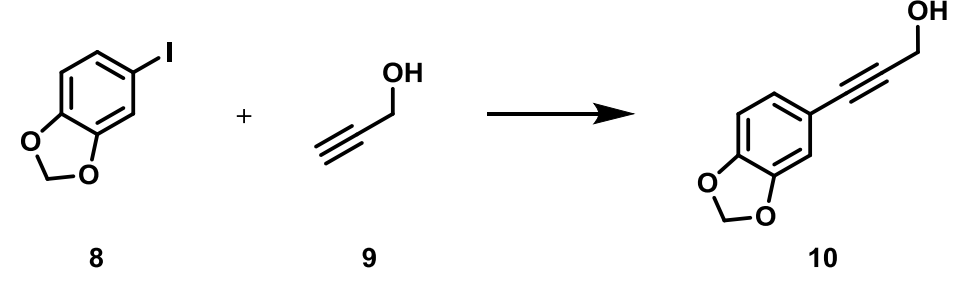

To a solution of 5-iodobenzo[ $d][1,3]$ dioxole $(8)(200 \mathrm{mg}, 0.806 \mathrm{mmol})$ in THF $(5 \mathrm{~mL})$ at room temperature was added sequentially $\mathrm{Pd}\left(\mathrm{PPh}_{3}\right)_{2} \mathrm{Cl}_{2}(28.3 \mathrm{mg}, 40.3 \mu \mathrm{mol}), \mathrm{CuI}(3.85 \mathrm{mg}, 20.2 \mu \mathrm{mol})$, diisopropylamine $(0.797 \mathrm{~mL}, 5.64 \mathrm{mmol})$ and propargyl alcohol (9) $(52 \mu \mathrm{L}, 0.887 \mathrm{mmol})$. The reaction was stirred for $3 \mathrm{~h}$, then diluted with $\mathrm{Et}_{2} \mathrm{O}(15 \mathrm{~mL})$ and filtered through a pad of Celite ${ }^{\circledR}$, washing with $\mathrm{Et}_{2} \mathrm{O}(5 \mathrm{~mL})$. The filtrate was washed with sat aq $\mathrm{NH}_{4} \mathrm{Cl}(5 \mathrm{~mL})$, sat aq $\mathrm{NaHCO}_{3}(5 \mathrm{~mL})$ and brine $(5 \mathrm{~mL})$, then dried over anhydrous $\mathrm{Na}_{2} \mathrm{SO}_{4}$ and concentrated in vacuo. Purification by flash column chromatography (50\% EtOAc/Hex) on silica gel afforded the title compound (127 $\mathrm{mg}, 89 \%)$ as a colourless solid; $\mathbf{R}_{f} 0.29$ (33\% EtOAc/Hex); $\mathbf{m p} 69-71{ }^{\circ} \mathrm{C}\left(\mathrm{CH}_{2} \mathrm{Cl}_{2} /\right.$ petrol); $\mathbf{v}_{\max } / \mathrm{cm}^{-1}: 3335$ (br), 2988 (s), 2901 (s), 1487 (s), 1247 (s), 1208 (s); ${ }^{1} \mathbf{H}$ NMR (400 MHz, CDCl $) \delta 6.96(1 \mathrm{H}, \mathrm{dt}, J=8.0,1.0 \mathrm{~Hz})$, $6.88(1 \mathrm{H}, \mathrm{s}), 6.75(1 \mathrm{H}, \mathrm{dd}, J=8.0,1.0 \mathrm{~Hz}), 5.97(2 \mathrm{H}, \mathrm{d}, J=1.0 \mathrm{~Hz}), 4.47(2 \mathrm{H}, \mathrm{s}) ;{ }^{13} \mathbf{C} \mathbf{N M R}(101 \mathrm{MHz}$, $\left.\mathrm{CDCl}_{3}\right) \delta 148.2(\mathrm{C}), 147.5(\mathrm{C}), 126.6(\mathrm{CH}), 115.9(\mathrm{C}), 111.8(\mathrm{CH}), 108.6(\mathrm{CH}), 101.5\left(\mathrm{CH}_{2}\right), 85.74(\mathrm{C})$, $85.69(\mathrm{C}), 51.8\left(\mathrm{CH}_{2}\right)$; HRMS $\left(\mathrm{APCI}^{+}\right)$: Calculated for $\mathrm{C}_{10} \mathrm{H}_{9} \mathrm{O}_{3}:$ 177.0546. Found $[\mathrm{M}+\mathrm{H}]^{+}: 177.0542$.

(10): 3-(Benzo[d][1,3]dioxol-5-yl)prop-2-yn-1-yl propiolate

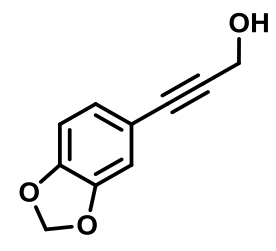

10

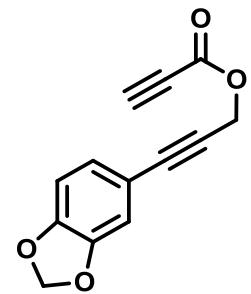

10

To a solution of alcohol $10(304 \mathrm{mg}, 1.73 \mathrm{mmol})$ and propiolic acid $(145 \mathrm{mg}, 2.07 \mathrm{mmol})$ in $\mathrm{CH}_{2} \mathrm{Cl}_{2}$ $(8.6 \mathrm{~mL})$ at $0{ }^{\circ} \mathrm{C}$ was added a solution of DCC (427 mg, $\left.2.07 \mathrm{mmol}\right)$ and DMAP (21.1 $\left.\mathrm{mg}, 0.173 \mathrm{mmol}\right)$ in $\mathrm{CH}_{2} \mathrm{Cl}_{2}(3.5 \mathrm{~mL})$ dropwise. The resulting mixture was stirred at $0{ }^{\circ} \mathrm{C}$ for $2 \mathrm{~h}$, then filtered through a pad of Celite ${ }^{\circledR}$ and the filtrate was concentrated in vacuo. Purification by flash column chromatography (20\% EtOAc/Hex) on silica gel afforded the title compound (388 mg, 99\%) as an off-white solid; $\mathbf{R}_{f}$ 0.66 (33\% EtOAc/Hex); mp 73-75 ${ }^{\circ} \mathrm{C}\left(\mathrm{CH}_{2} \mathrm{Cl}_{2} /\right.$ petrol); $\mathbf{v}_{\max } / \mathrm{cm}^{-1}: 3260$ (s), 2902 (m), 2235 (w), 2118 (s), 1707 (s), 1490 (s); ${ }^{1} \mathrm{H}$ NMR (400 MHz, $\left.\mathrm{CDCl}_{3}\right) \delta 6.99(1 \mathrm{H}, \mathrm{d}, J=8.0 \mathrm{~Hz}), 6.89(1 \mathrm{H}, \mathrm{s}), 6.75(1 \mathrm{H}$, 
$\mathrm{d}, J=8.0 \mathrm{~Hz}), 5.98(2 \mathrm{H}, \mathrm{s}), 4.98(2 \mathrm{H}, \mathrm{s}), 2.95(1 \mathrm{H}, \mathrm{s}) ;{ }^{13} \mathbf{C ~ N M R}\left(101 \mathrm{MHz}, \mathrm{CDCl}_{3}\right) \delta 152.1(\mathrm{C}), 148.6$

(C), $147.6(\mathrm{C}), 127.0(\mathrm{CH}), 115.1(\mathrm{C}), 112.0(\mathrm{CH}), 108.6(\mathrm{CH}), 101.5\left(\mathrm{CH}_{2}\right), 87.6(\mathrm{C}), 80.1(\mathrm{C}), 75.9$ $(\mathrm{CH}), 74.2(\mathrm{C}), 54.6\left(\mathrm{CH}_{2}\right)$; HRMS $\left(\mathrm{APCI}^{+}\right)$: Calculated for $\mathrm{C}_{13} \mathrm{H}_{9} \mathrm{O}_{4}: 229.0495$. Found $[\mathrm{M}+\mathrm{H}]^{+}$: 229.0489.

\section{Justicidone}

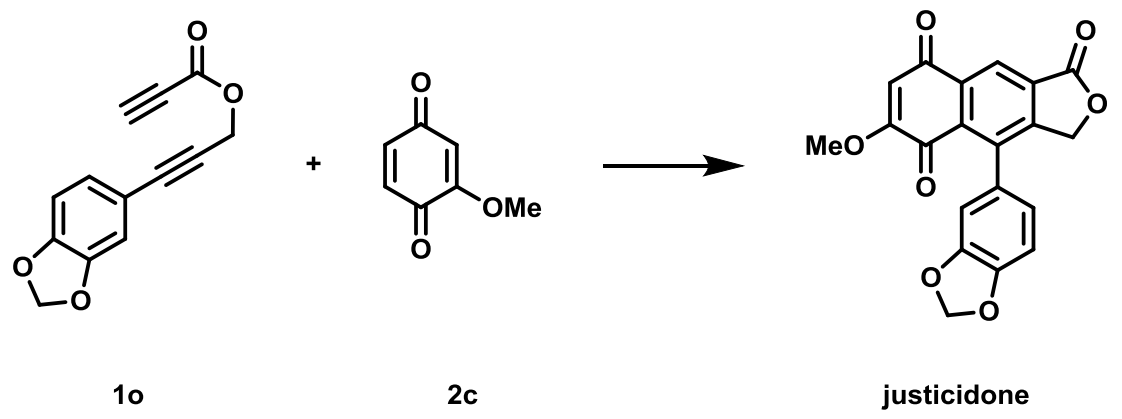

A reaction tube was charged with 2-methoxybenzoquinone (2c) $(41.4 \mathrm{mg}, 300 \mu \mathrm{mol}),\left(4-\mathrm{NCC}_{6} \mathrm{H}_{4}\right)_{3} \mathrm{P}$ $(5.05 \mathrm{mg}, 15.0 \mu \mathrm{mol})$ and $\left[\mathrm{Rh}(\mathrm{coe})_{2} \mathrm{Cl}\right]_{2}(2.69 \mathrm{mg}, 3.75 \mu \mathrm{mol})$. The tube was flushed with argon, then fitted with a rubber septum, placed under a balloon of argon and DCE $(0.25 \mathrm{~mL})$ was added via syringe. The reaction tube was placed in a preheated heating block at $70{ }^{\circ} \mathrm{C}$, then diyne $1 \mathrm{o}(22.8 \mathrm{mg}, 100 \mu \mathrm{mol})$ in DCE $(0.75 \mathrm{~mL})$ was added dropwise by syringe pump over $2 \mathrm{~h}$. After addition of the diyne 10 was complete, the reaction mixture was stirred at $70{ }^{\circ} \mathrm{C}$ for $1 \mathrm{~h}$, then cooled to room temperature and concentrated in vacuo. The residue obtained was partitioned between EtOAc $(10 \mathrm{~mL})$ and $\mathrm{H}_{2} \mathrm{O}(5 \mathrm{~mL})$. The organic layer was washed with $0.5 \mathrm{M} \mathrm{KOH}(2 \times 5 \mathrm{~mL})$, then the combined aqueous layers were extracted with EtOAc $(10 \mathrm{~mL})$. The combined organic layers were dried over anhydrous $\mathrm{Na}_{2} \mathrm{SO}_{4}$ and concentrated in vacuo. Purification by flash column chromatography (10\% acetone/PhMe) on silica gel afforded the title compound (15.6 mg, 42\%,r.r. >15:1) as an orange solid; $\mathbf{R}_{f} 0.29$ (20\% acetone/PhMe); mp 113-114 ${ }^{\circ} \mathrm{C}\left(\mathrm{CH}_{2} \mathrm{Cl}_{2} / \mathrm{Et}_{2} \mathrm{O}\right)$ [Lit., $114-115{ }^{\circ} \mathrm{C}, \mathrm{EtOAc} / \mathrm{Hex}$ ]; ${ }^{1} \mathbf{H} \mathbf{~ N M R}\left(400 \mathrm{MHz}, \mathrm{CDCl}_{3}\right) \delta 8.72$ $(1 \mathrm{H}, \mathrm{s}), 6.95-6.90(1 \mathrm{H}, \mathrm{m}), 6.64-6.60(2 \mathrm{H}, \mathrm{m}), 6.26(1 \mathrm{H}, \mathrm{s}), 6.05(2 \mathrm{H}, \mathrm{d}, J=9.5 \mathrm{~Hz}), 5.22-5.05(2 \mathrm{H}$, m), $3.88(3 \mathrm{H}, \mathrm{s}) ;{ }^{13} \mathrm{C}$ NMR (101 MHz, $\left.\mathrm{CDCl}_{3}\right) \delta 183.0(\mathrm{C}), 179.5$ (C), 169.3 (C), $161.2(\mathrm{C}), 151.5$ (C), 148.5 (C), 148.1 (C), 138.8 (C), 135.2 (C), 131.9 (C), 129.9 (C), 129.3 (C), $124.1(\mathrm{CH}), 120.5(\mathrm{CH})$, $109.3(\mathrm{CH}), 109.1(\mathrm{CH}), 108.0(\mathrm{CH}), 101.6\left(\mathrm{CH}_{2}\right), 69.8\left(\mathrm{CH}_{2}\right), 56.9\left(\mathrm{CH}_{3}\right)$; The spectroscopic properties were consistent with the data available in the literature. ${ }^{31}$ 


\section{Copies of ${ }^{1} \mathrm{H}$ and ${ }^{13} \mathrm{C}$ NMR Spectra for Novel Compounds}

4,4',4'-Phosphanetriyltribenzonitrile

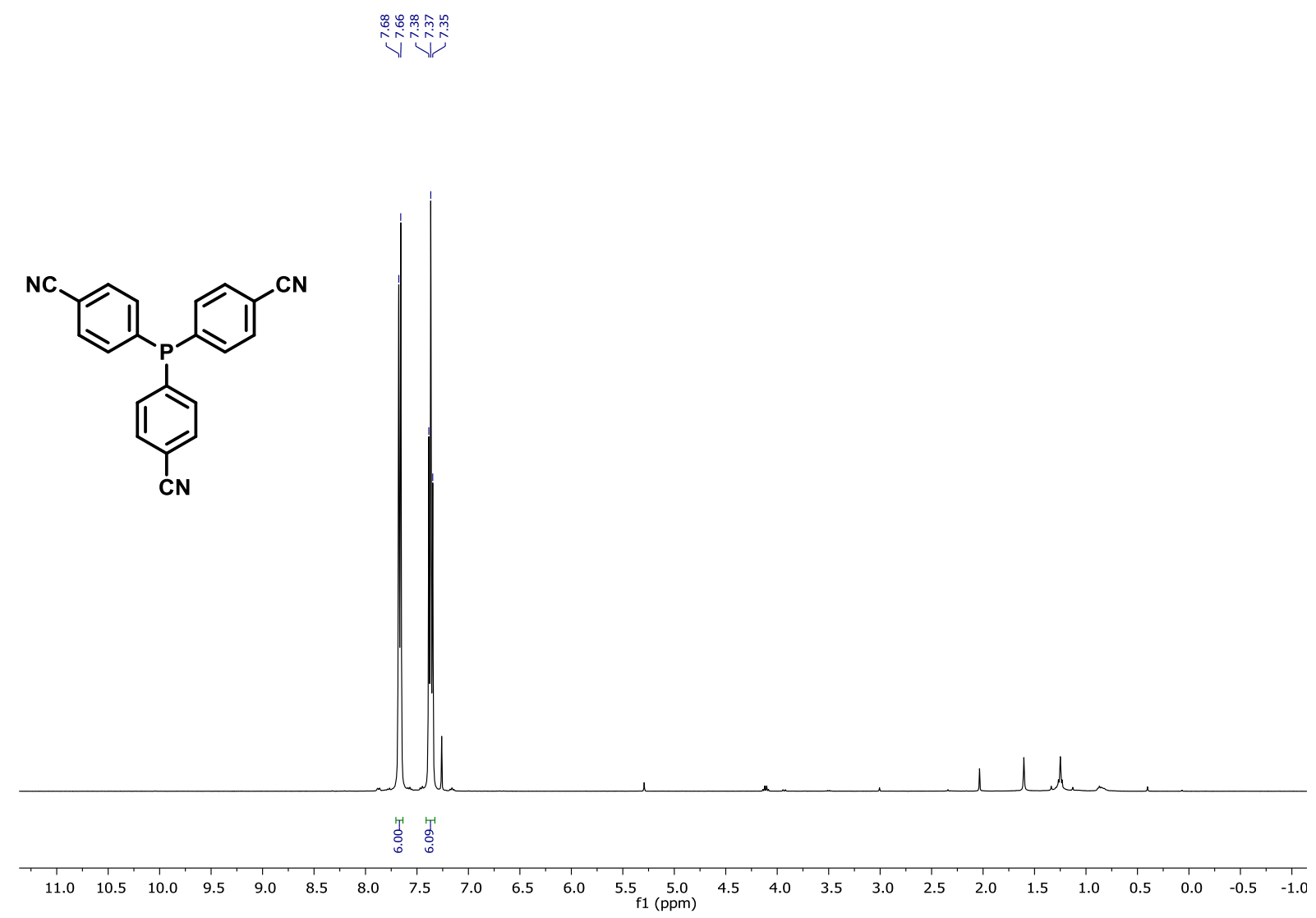


(1f): $N, N$-Di(hept-2-yn-1-yl)-4-methylbenzenesulfonamide

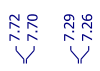

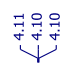

亭设

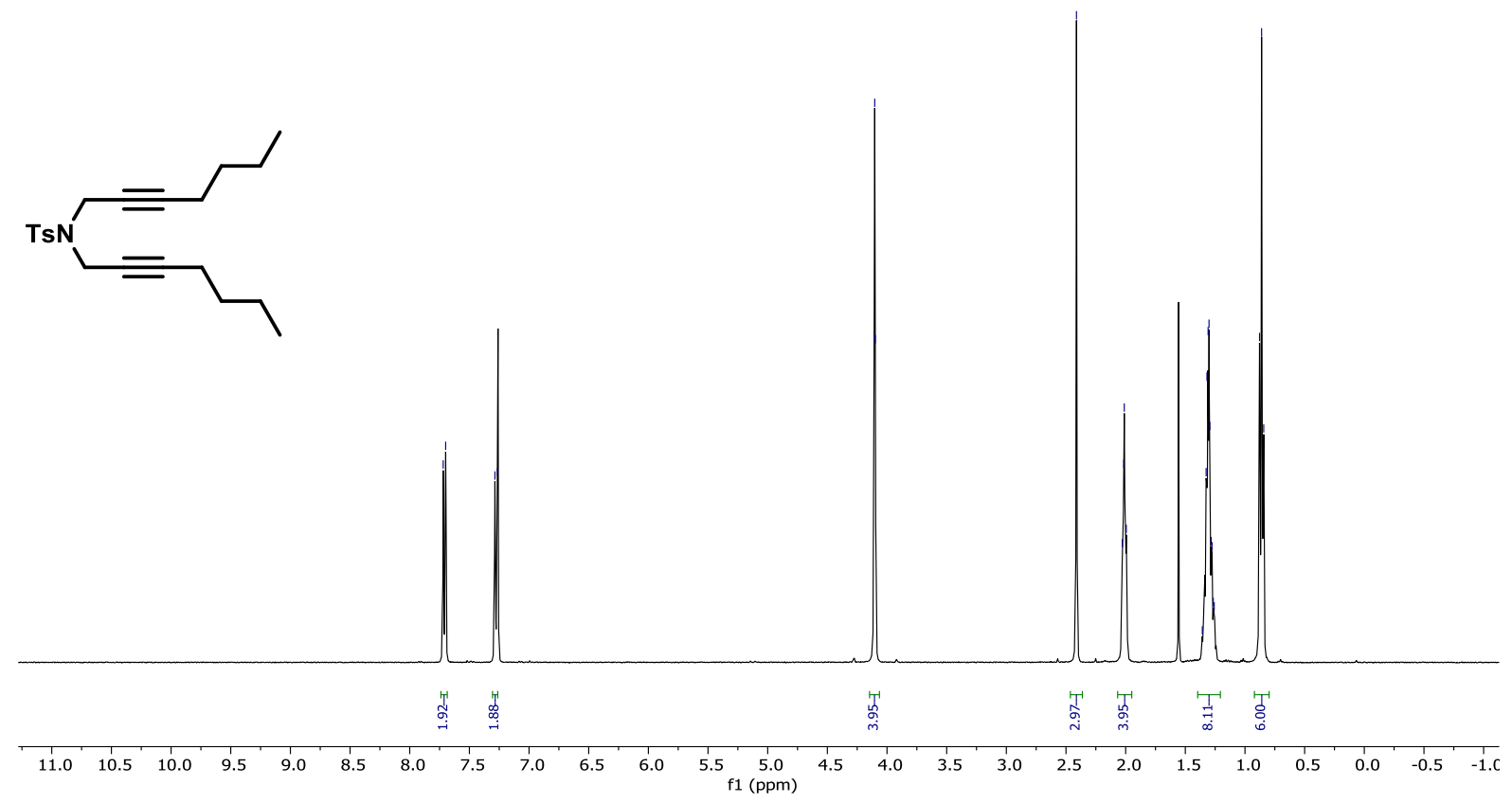

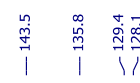

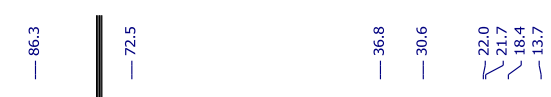

$\begin{array}{lllllllllll}210 & 200 & 190 & 180 & 170 & 160 & 150 & 140 & 130 & 120 & 110 \\ \mathrm{f} 1(\mathrm{ppm}) & 100\end{array}$

80

60

40

$\begin{array}{lllll}10 & 10 & 0 & -10\end{array}$ 
(1h): Dimethyl 2-(hept-2-yn-1-yl)-2-(prop-2-yn-1-yl)malonate

范

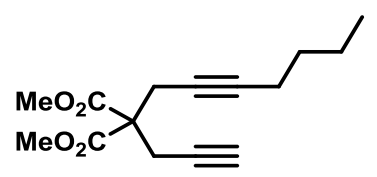

$\mathrm{MeO}_{2} \mathrm{C}=$
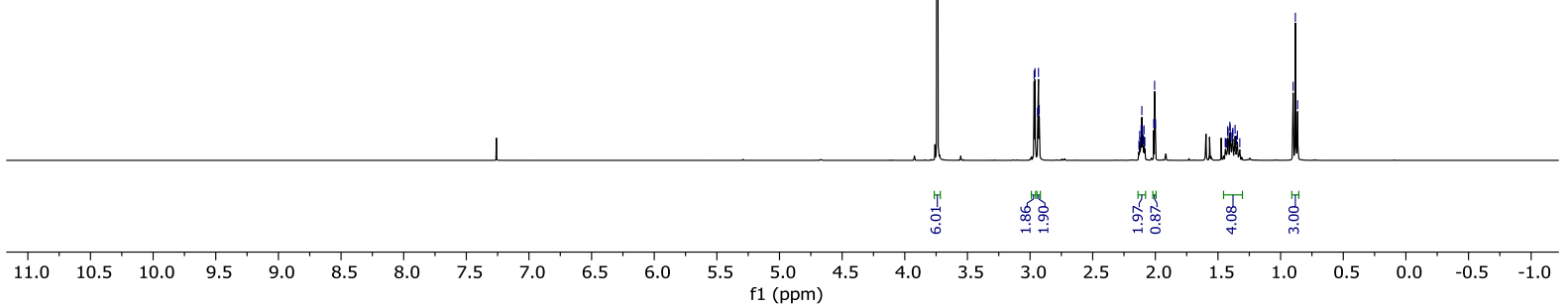
(S4): (E)- $N$-(1,2-Dichlorovinyl)-4-methyl- $N$-(2-(phenylethynyl)phenyl)benzenesulfonamide

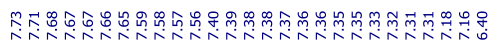<smiles>CN(/C(Cl)=C\Cl)c1ccccc1C#Cc1ccccc1</smiles>

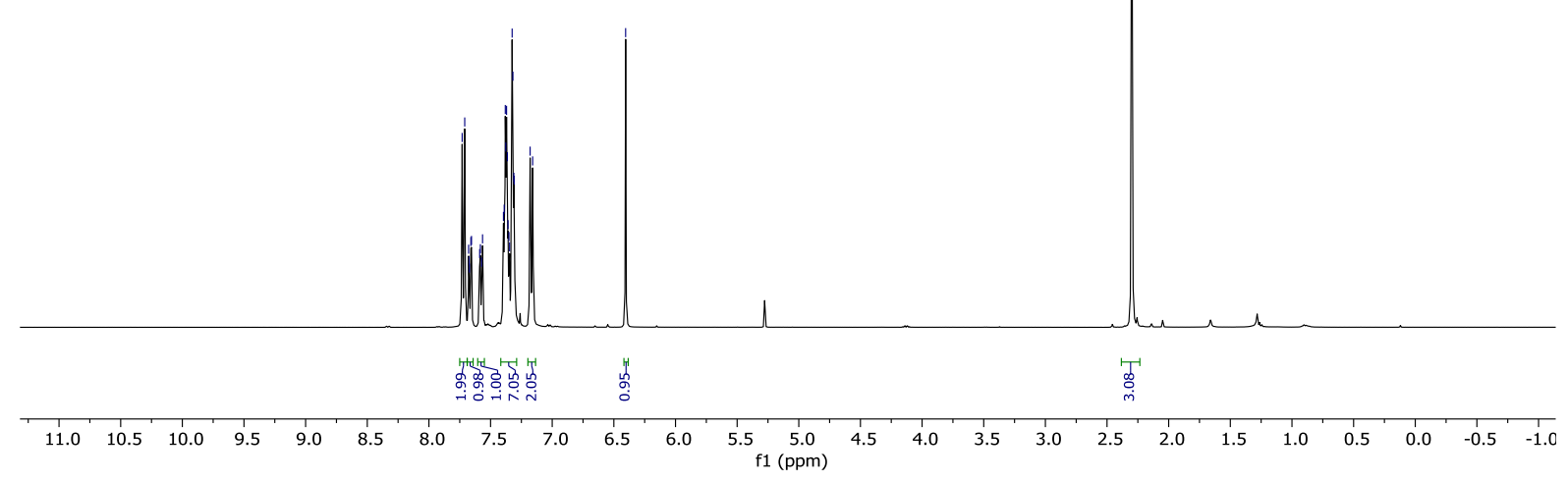

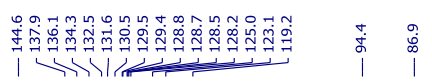

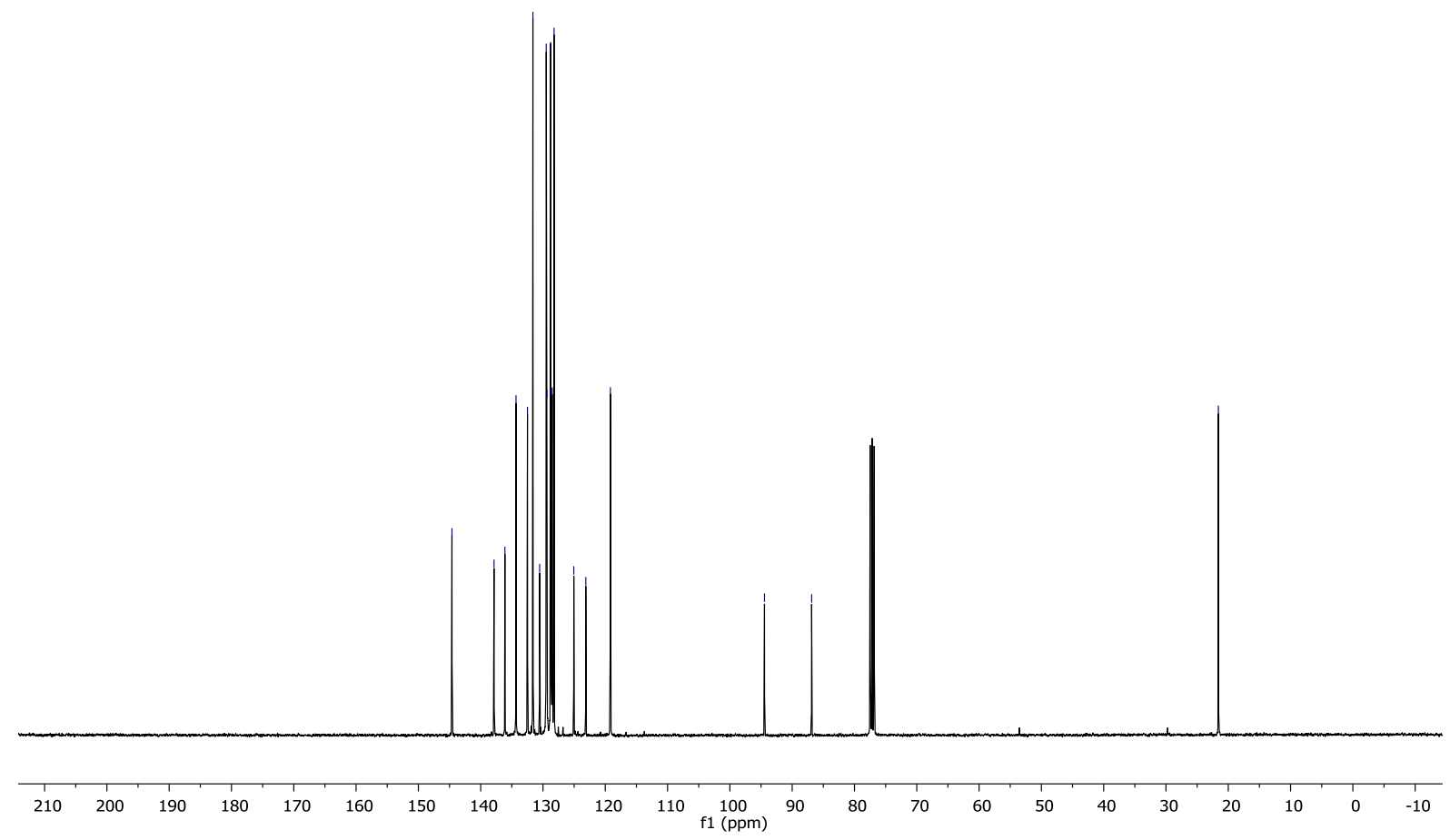


(1m): $N$-Ethynyl-4-methyl- $N$-(2-(phenylethynyl)phenyl)benzenesulfonamide

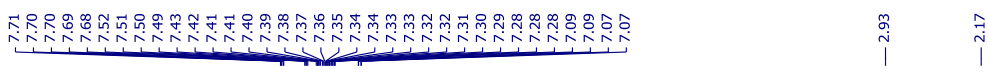<smiles>C#CN(C)c1ccccc1C#Cc1ccccc1</smiles>

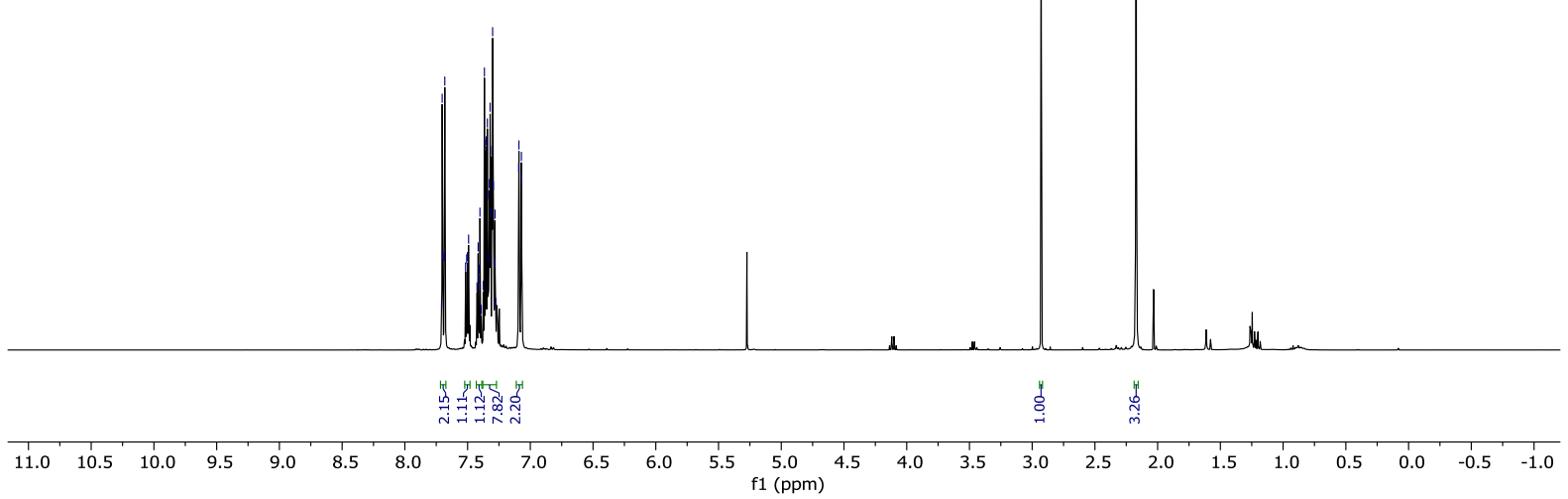


(2f): 2-Iodo-1,4-benzoquinone<smiles>O=C1C=CC(=O)C(I)=C1</smiles>

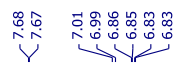

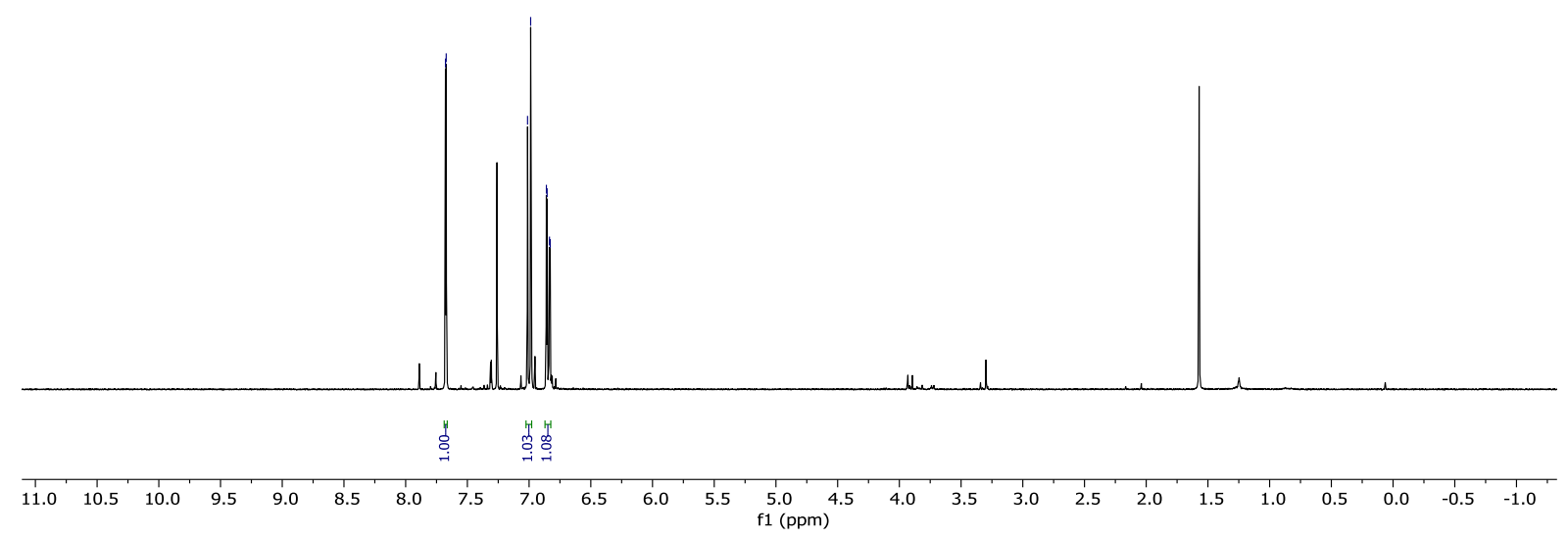

(2i): 2-(2-Bromophenyl)-1,4-benzoquinone<smiles>O=C1C=CC(=O)C(c2ccccc2Br)=C1</smiles>

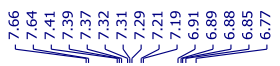

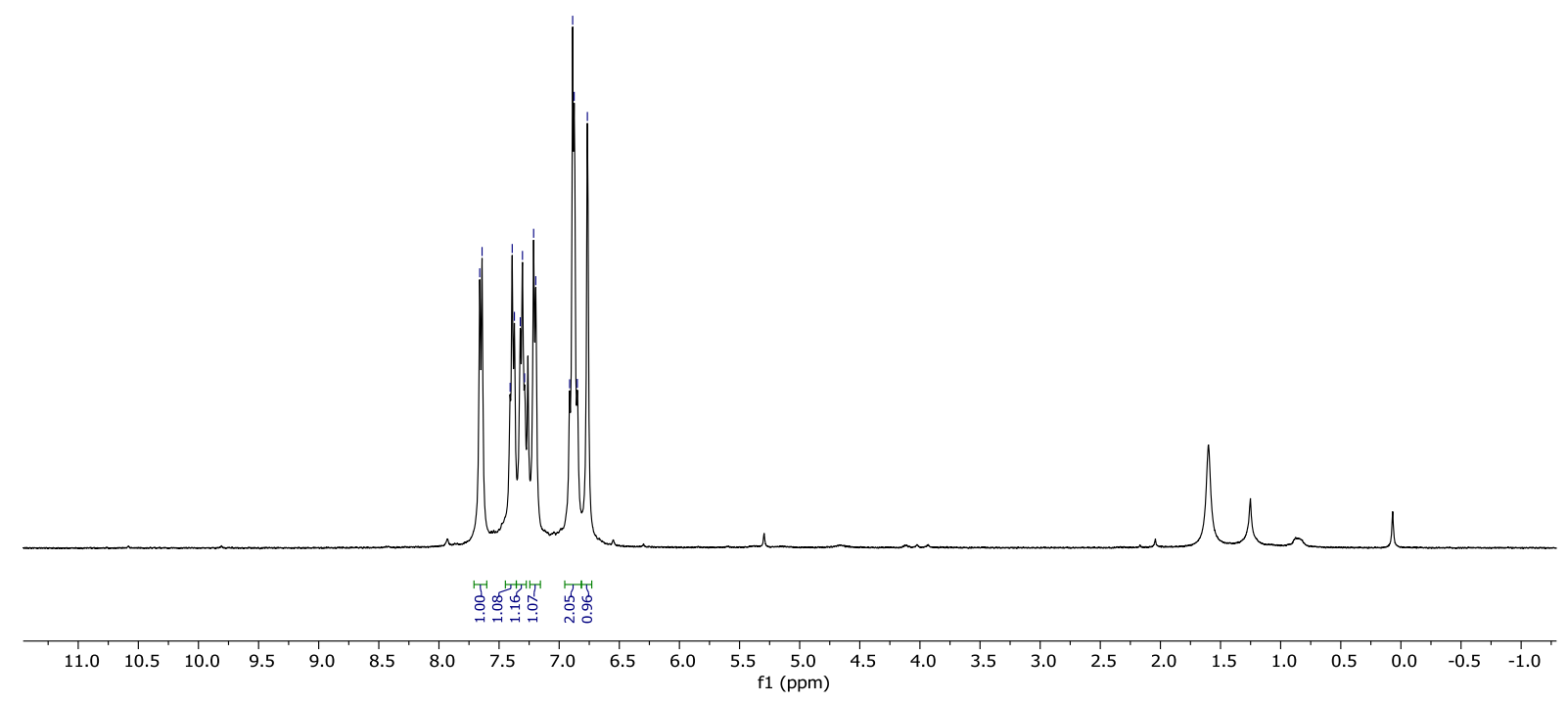


(2k): 2-(Methyl(phenyl)amino)-1,4-benzoquinone

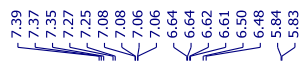

$\prod_{0}^{m}$

(3aa): Dimethyl 5,8-dioxo-1,3,5,8-tetrahydro-2H-cyclopenta[b]naphthalene-2,2-dicarboxylate

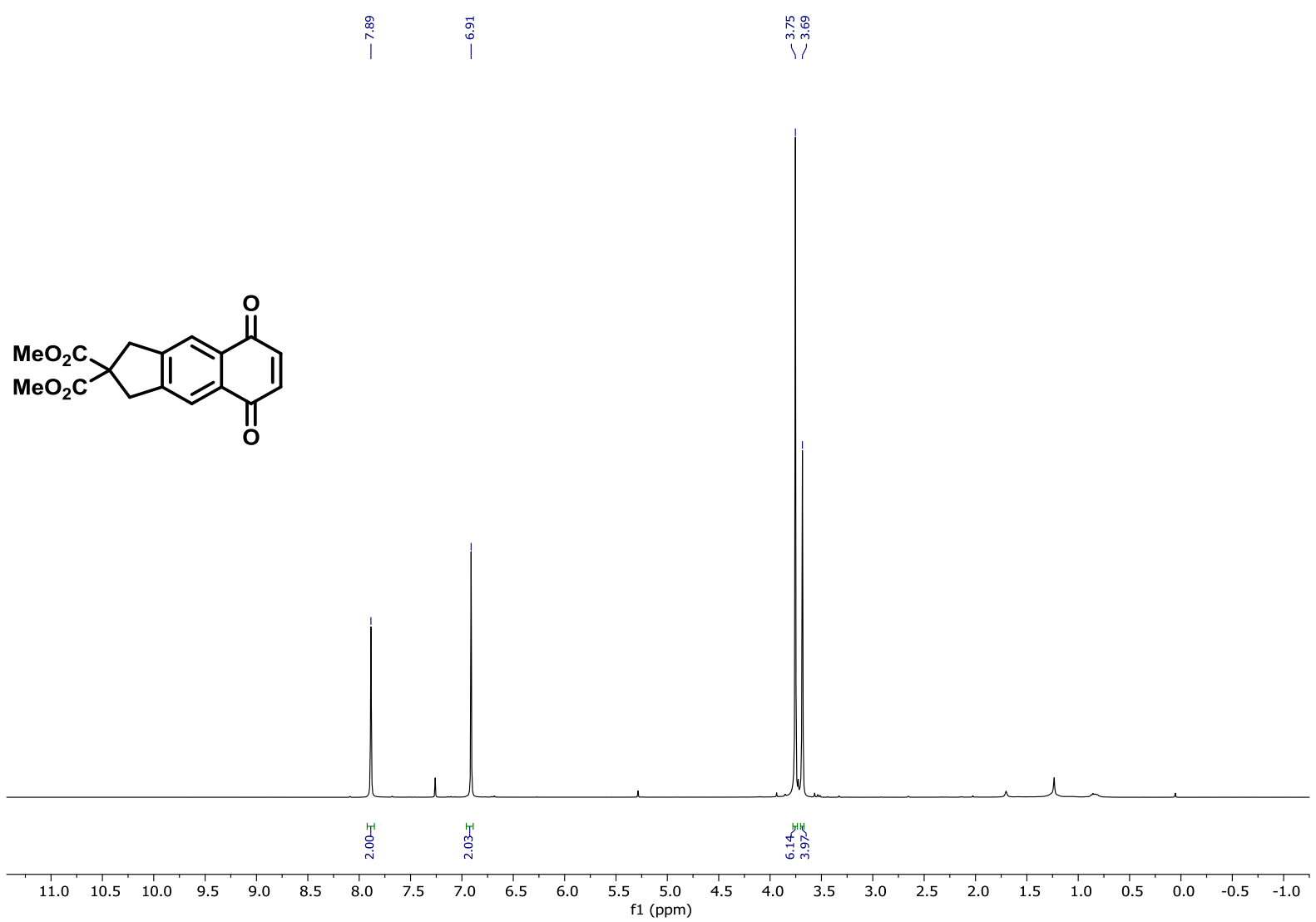


(3ab): Dimethyl 6,7-dimethyl-5,8-dioxo-1,3,5,8-tetrahydro-2H-cyclopenta[b]naphthalene-2,2dicarboxylate
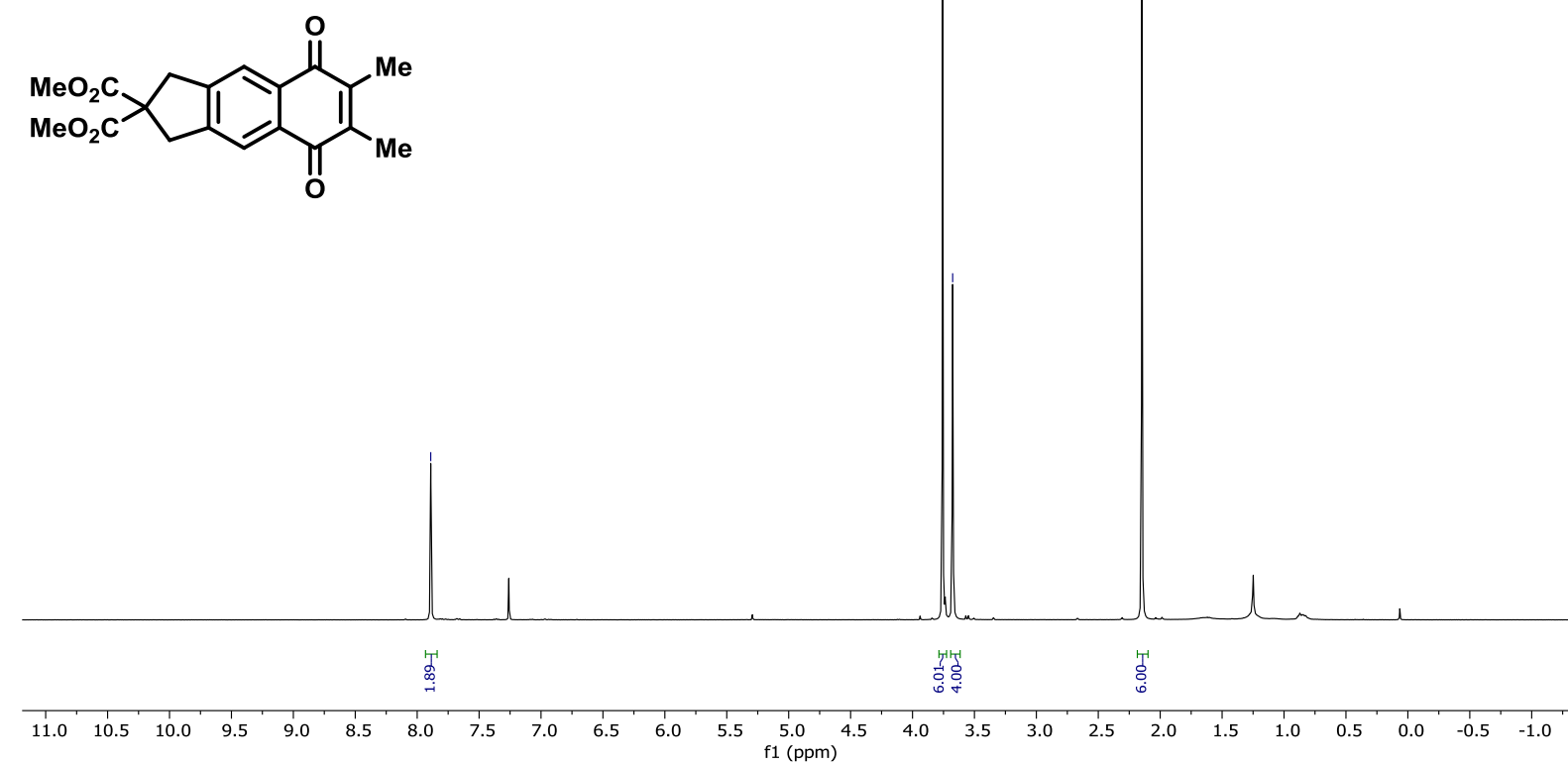

|일

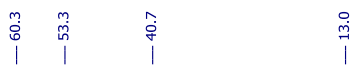

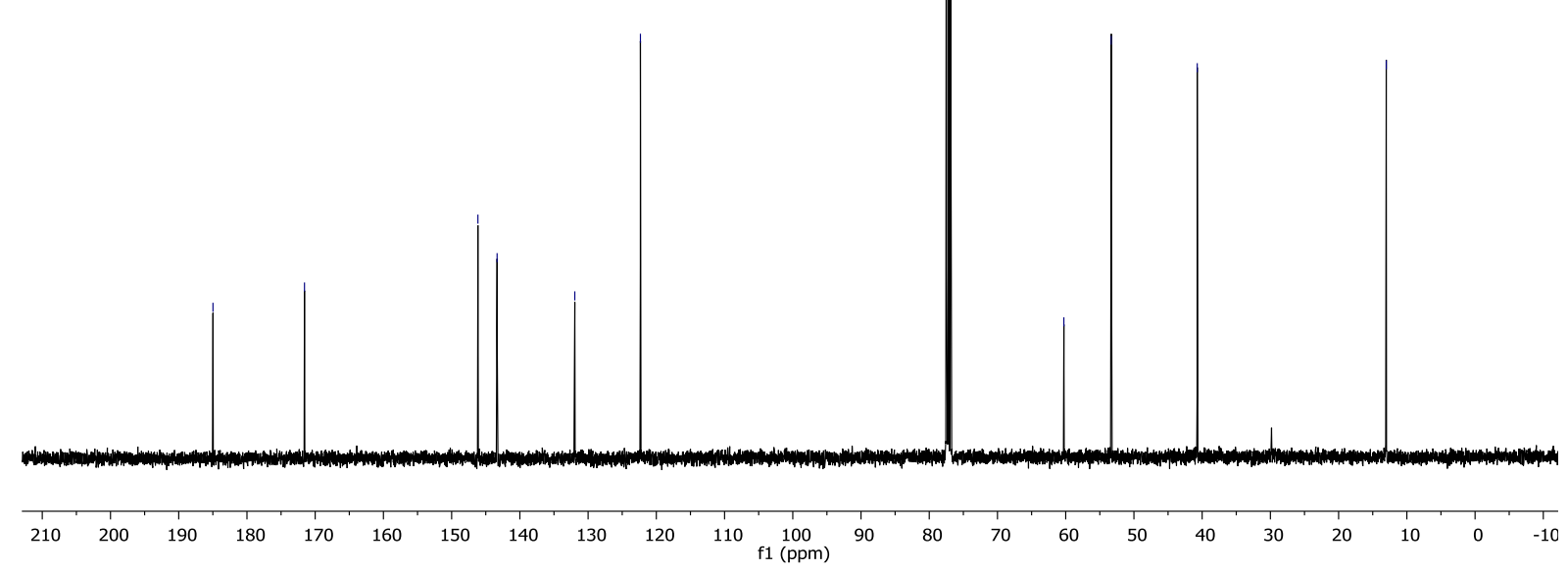


(3ac): Dimethyl 5,8-dioxo-6-methoxy-1,3,5,8-tetrahydro-2H-cyclopenta[b]naphthalene-2,2dicarboxylate

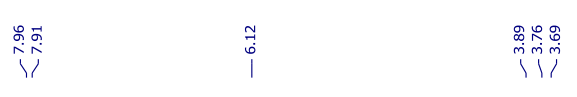

$\mathrm{MeO}_{\mathrm{MeO}_{2} \mathrm{C}} \mathrm{C}$

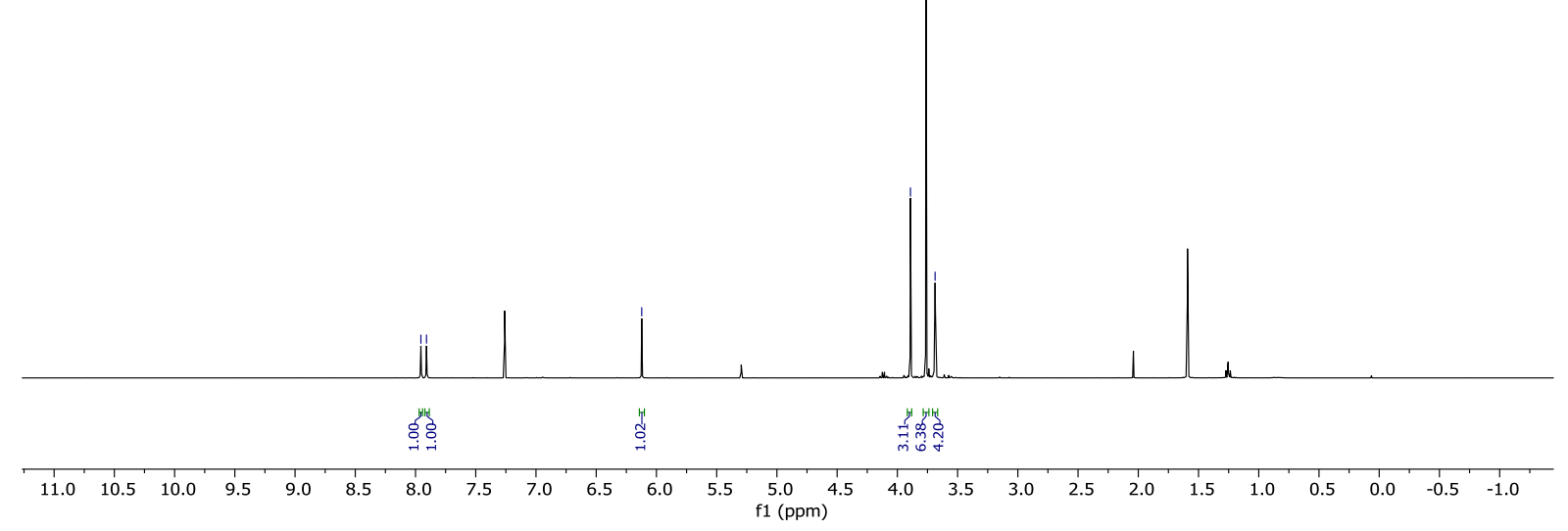

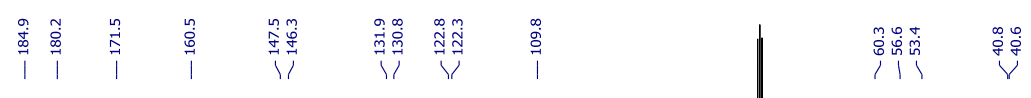

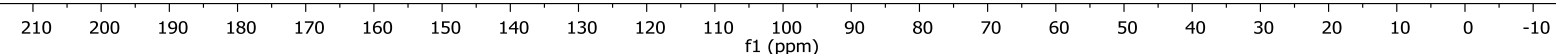


(3ad): Dimethyl 6-chloro-5,8-dioxo-1,3,5,8-tetrahydro-2H-cyclopenta[b]naphthalene-2,2dicarboxylate

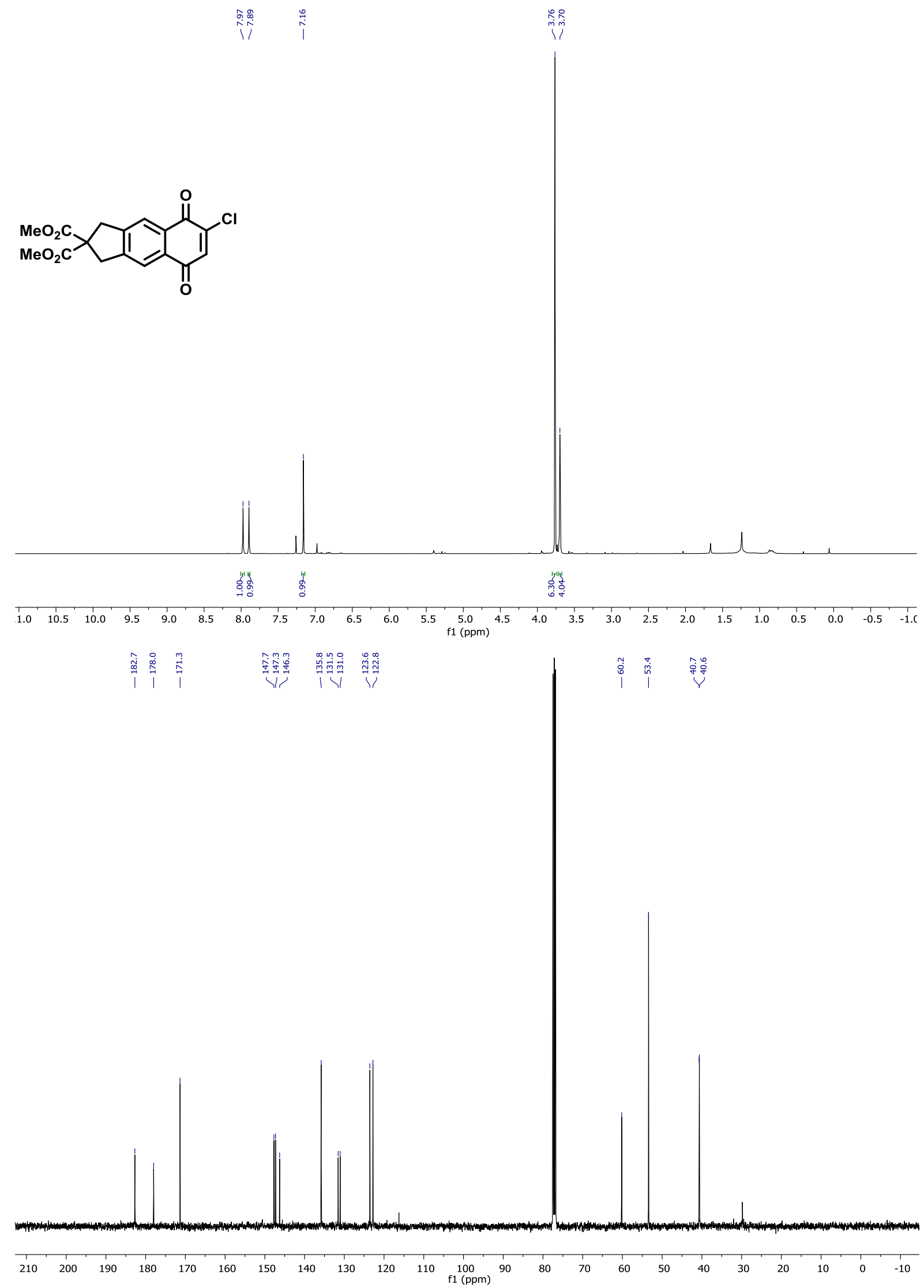


(3ae): Dimethyl 6-bromo-5,8-dioxo-1,3,5,8-tetrahydro-2H-cyclopenta[b]naphthalene-2,2dicarboxylate

în

ํㅐำ
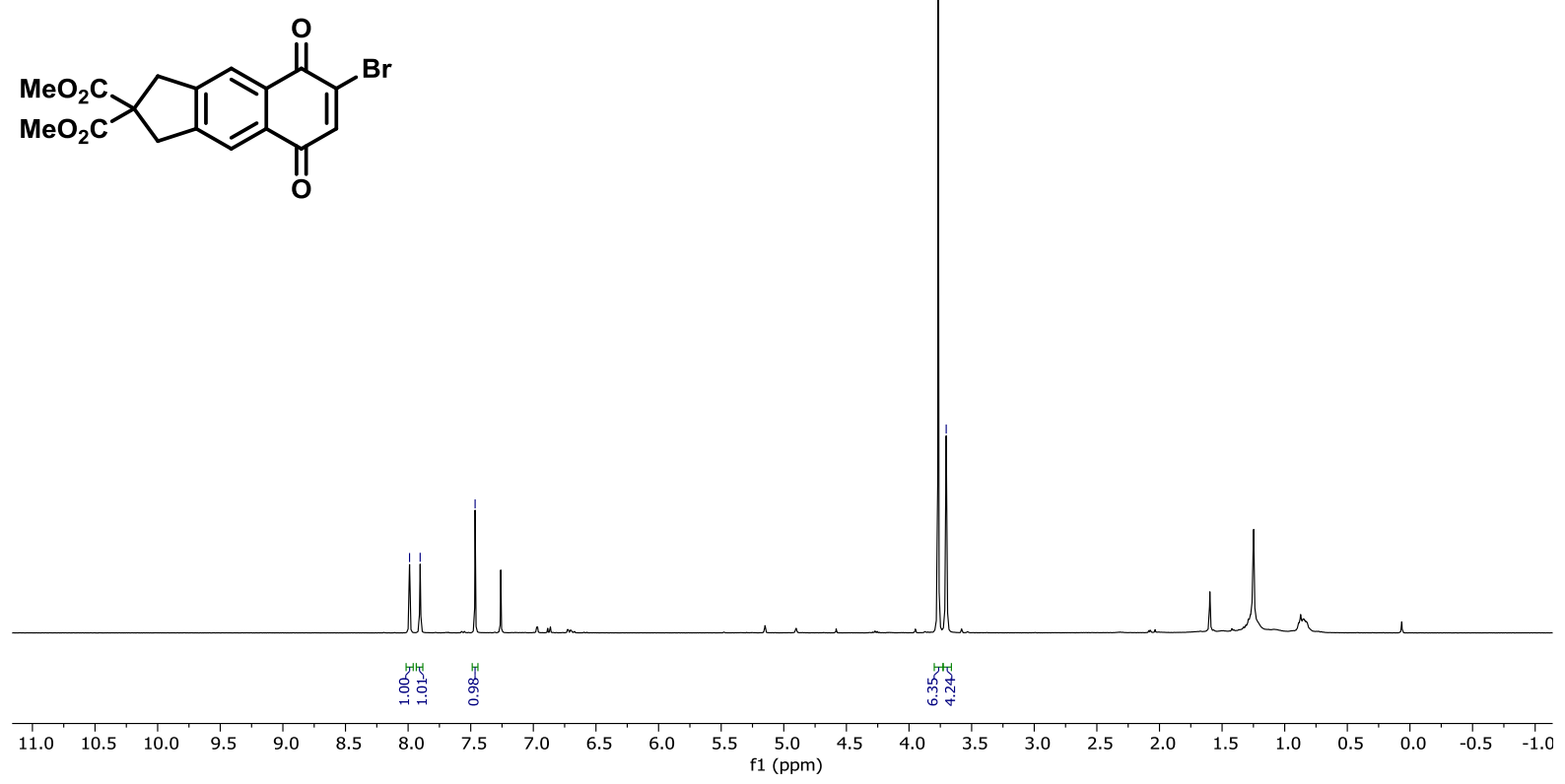

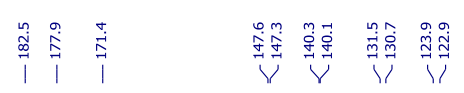

I:

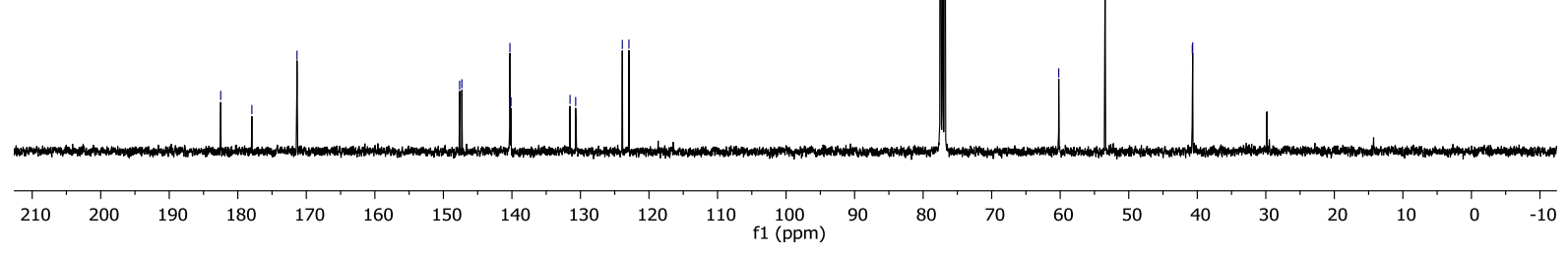

S43 
(3af): Dimethyl 6-iodo-5,8-dioxo-1,3,5,8-tetrahydro-2H-cyclopenta[b]naphthalene-2,2dicarboxylate<smiles>COC(C)(C)C1Cc2cc3c(cc2C1)C(=O)C(I)=CC3=O</smiles>

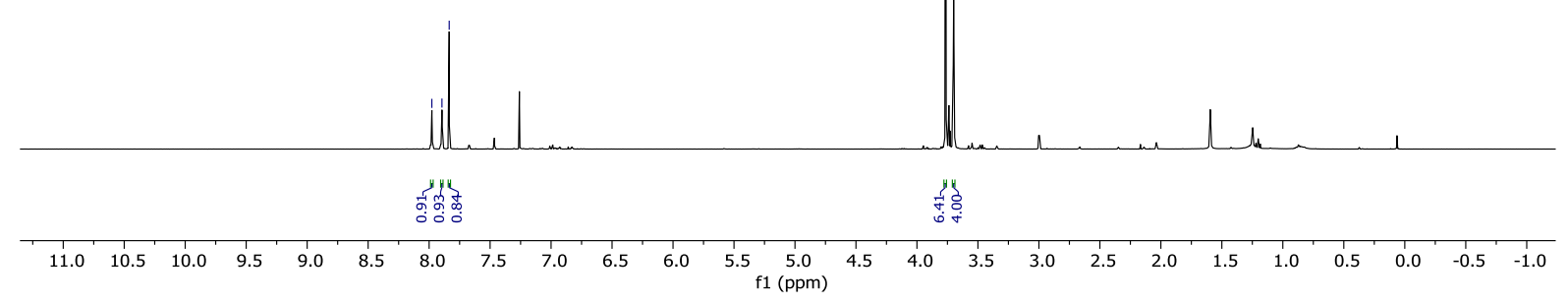

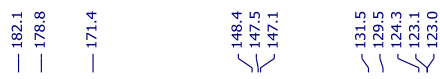

กับ

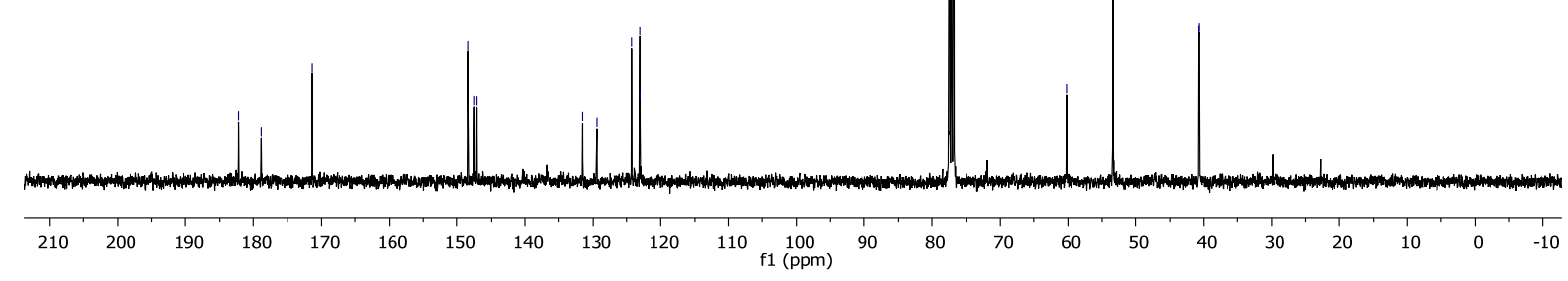


(3ag): Dimethyl 5,8-dioxo-6-phenyl-1,3,5,8-tetrahydro-2H-cyclopenta[b]naphthalene-2,2dicarboxylate

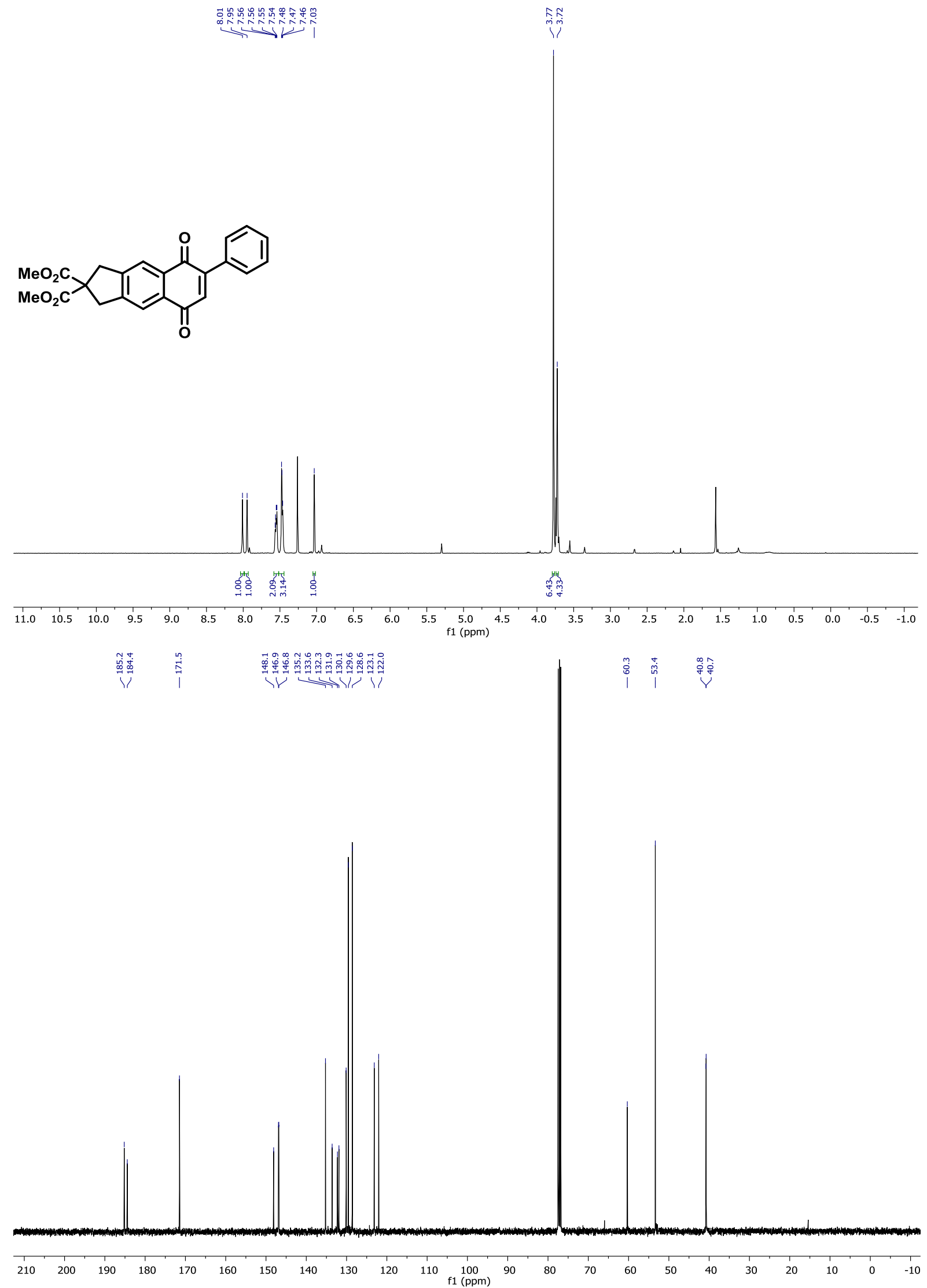


(3ah): Dimethyl 6-(2-methoxyphenyl)-5,8-dioxo-1,3,5,8-tetrahydro-2H-

cyclopenta[b]naphthalene-2,2-dicarboxylate

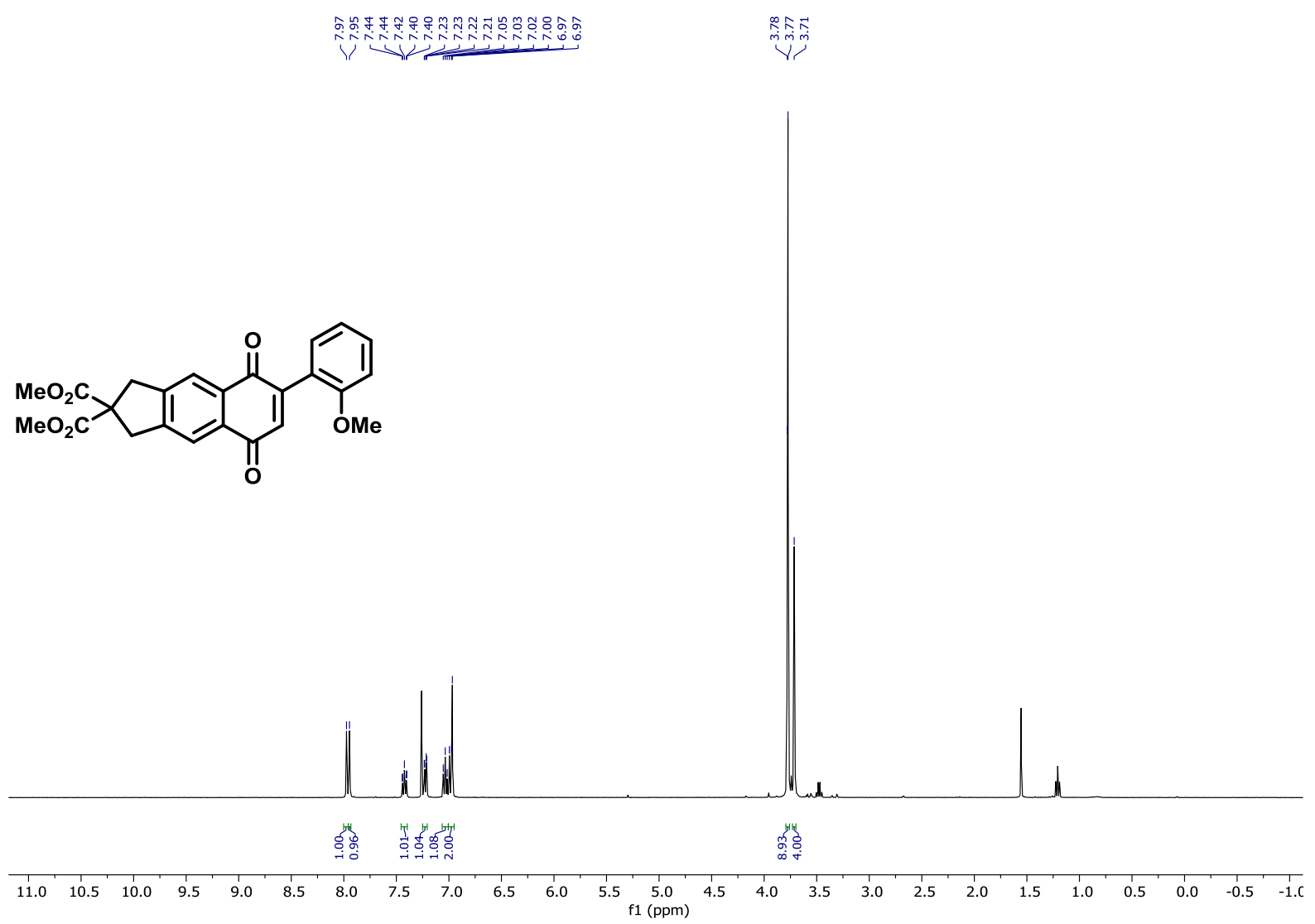

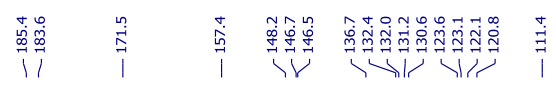

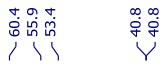

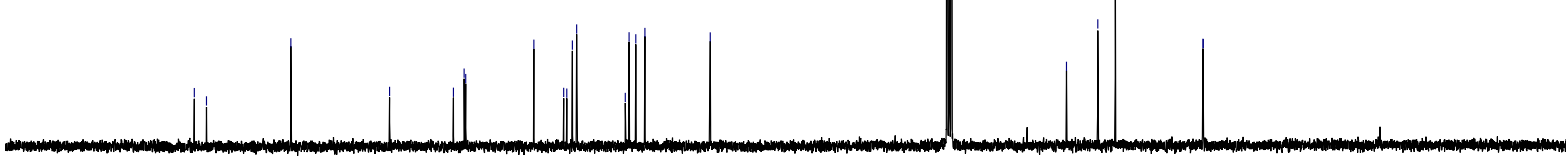

\begin{tabular}{lllllllllllllllllllllll}
\hline 210 & 200 & 190 & 180 & 170 & 160 & 150 & 140 & 130 & 120 & 110 & 100 & 90 & 80 & 70 & 60 & 50 & 40 & 30 & 20 & 10 & 0 & -10
\end{tabular} 
(3ai): Dimethyl 6-(2-bromophenyl)-5,8-dioxo-1,3,5,8-tetrahydro-2H-cyclopenta[b]naphthalene2,2-dicarboxylate

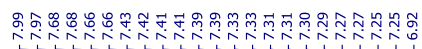

MeO

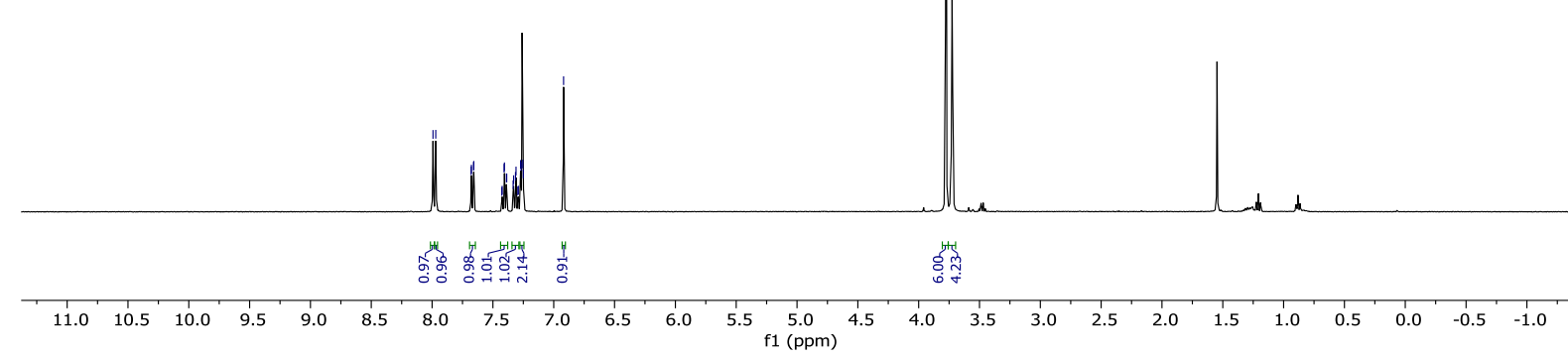

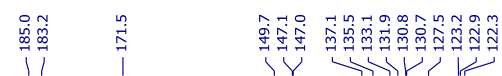

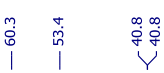

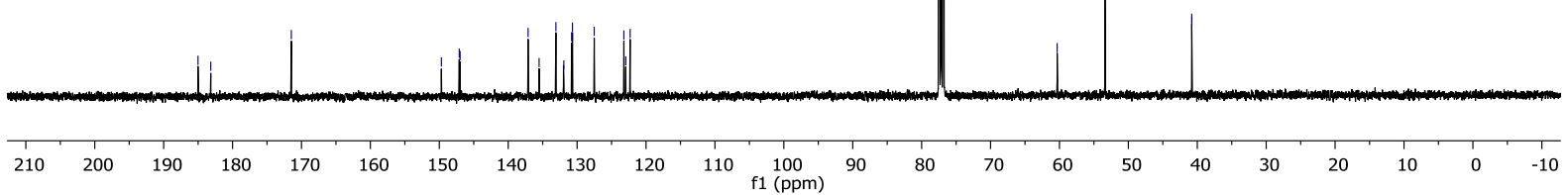


(3aj): Dimethyl 5,8-dioxo-6-(4-(trifluoromethyl)phenyl)-1,3,5,8-tetrahydro-2Hcyclopenta[$[b]$ naphthalene-2,2-dicarboxylate
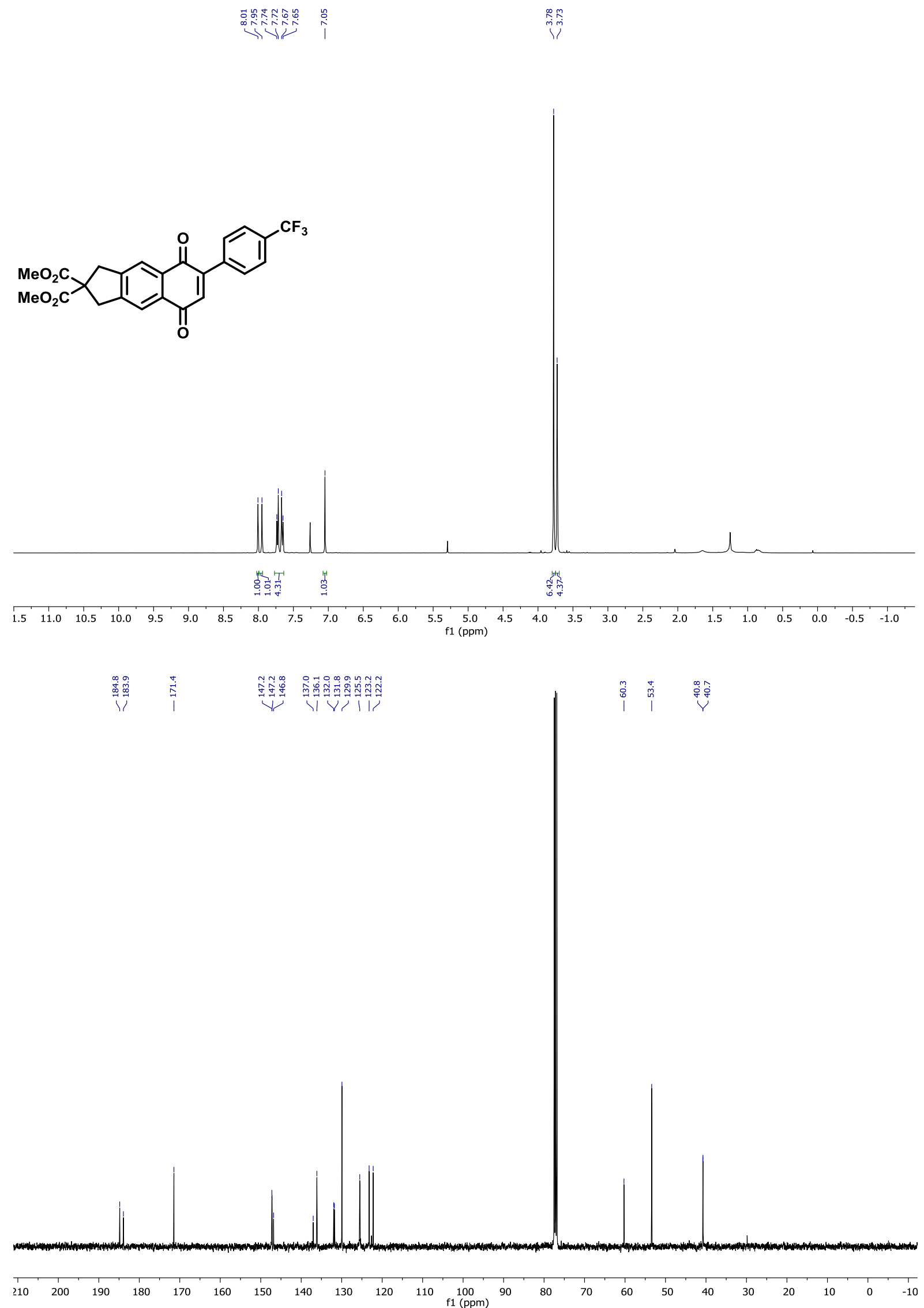
(3ak): Dimethyl 6-(methyl(phenyl)amino)-5,8-dioxo-1,3,5,8-tetrahydro-2H-

cyclopenta $[b]$ naphthalene-2,2-dicarboxylate

盗

$\mathrm{MeO}_{\mathrm{MeO}_{2} \mathrm{C}} \mathrm{C}$

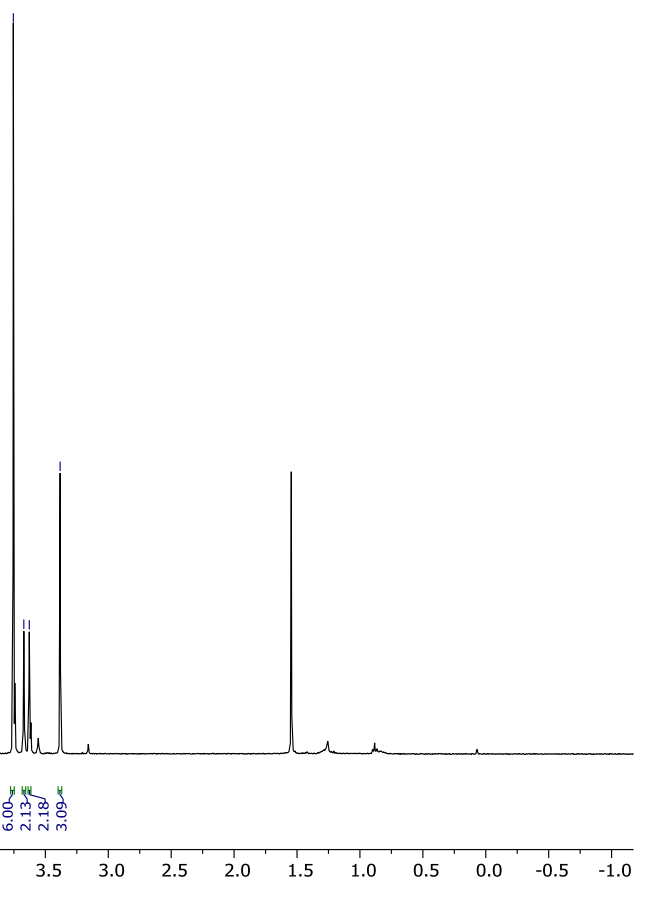

\begin{tabular}{|c|c|c|c|c|c|c|c|c|c|c|c|c|c|c|c|c|c|c|c|c|c|c|c|c|}
\hline \multirow[t]{2}{*}{11.0} & 10.5 & 10.0 & 9.5 & 9.0 & 8.5 & 8.0 & 7.5 & 7.0 & 6.5 & 6.0 & 5.5 & $\begin{array}{c}5.0 \\
1(\mathrm{ppm})\end{array}$ & 4.5 & 4.0 & 3.5 & 3.0 & 2.5 & 2.0 & 1.5 & 1.0 & 0.5 & & \multirow[t]{2}{*}{-0.5} & -1.0 \\
\hline & & & 金 & نُ & & & 年 & & 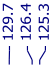 & & $\underset{\vec{\Xi}}{\vec{\Xi}}$ & & & & & 1 & $\stackrel{m}{\stackrel{m}{n}}$ & $\ddot{m} \dot{\gamma}$ & & & & & & \\
\hline
\end{tabular}


(3al): Dimethyl 5,10-dioxo-1,3,5,10-tetrahydro-2H-cyclopenta[b]anthracene-2,2-dicarboxylate

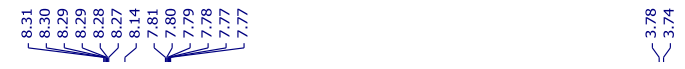<smiles>CC(=O)C1(C(C)=O)Cc2cc3c(cc2C1)C(=O)c1ccccc1C3=O</smiles>
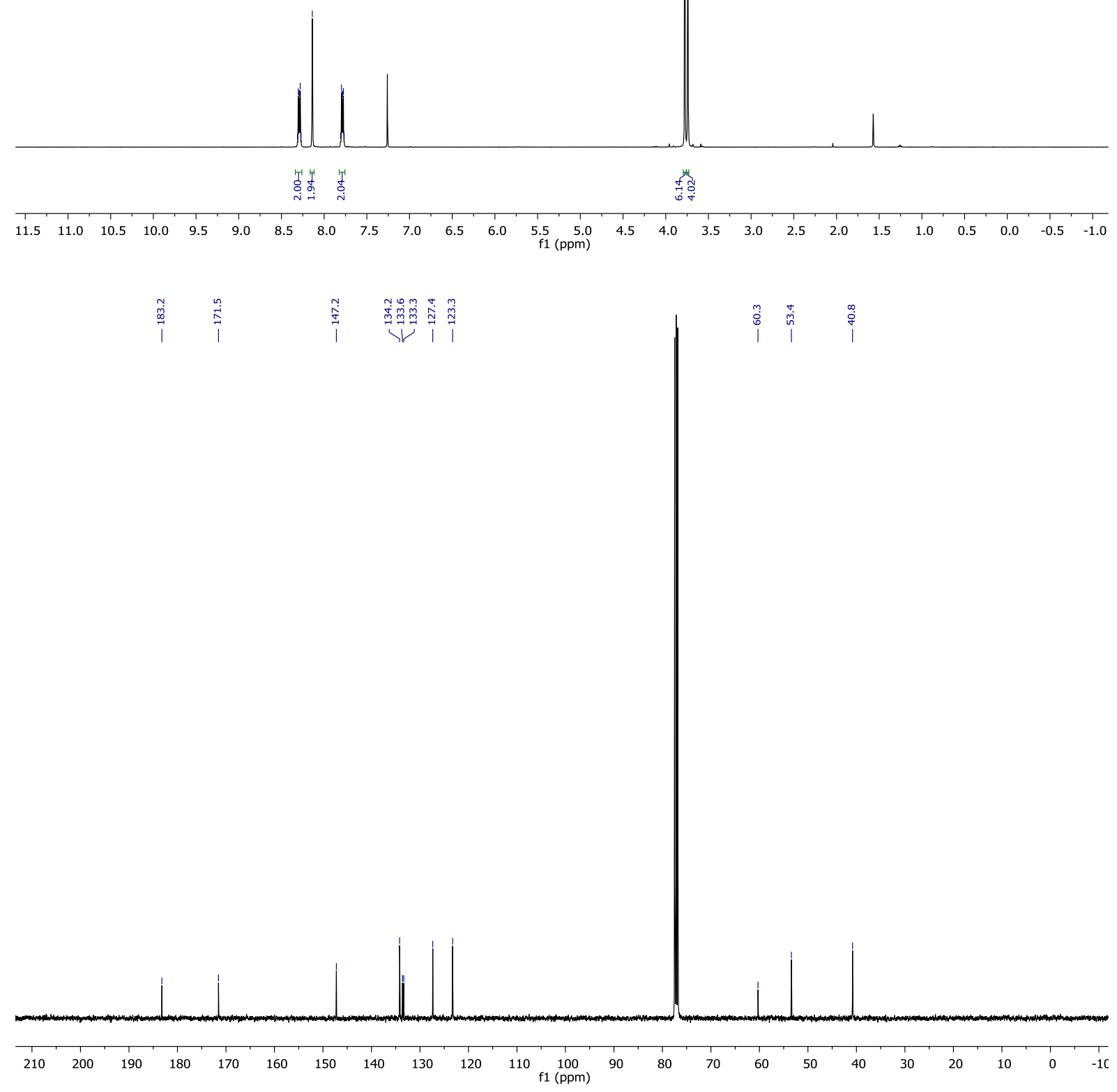
(3ba): 2,3-Dihydro-1H-cyclopenta[b]naphthalene-5,8-dione

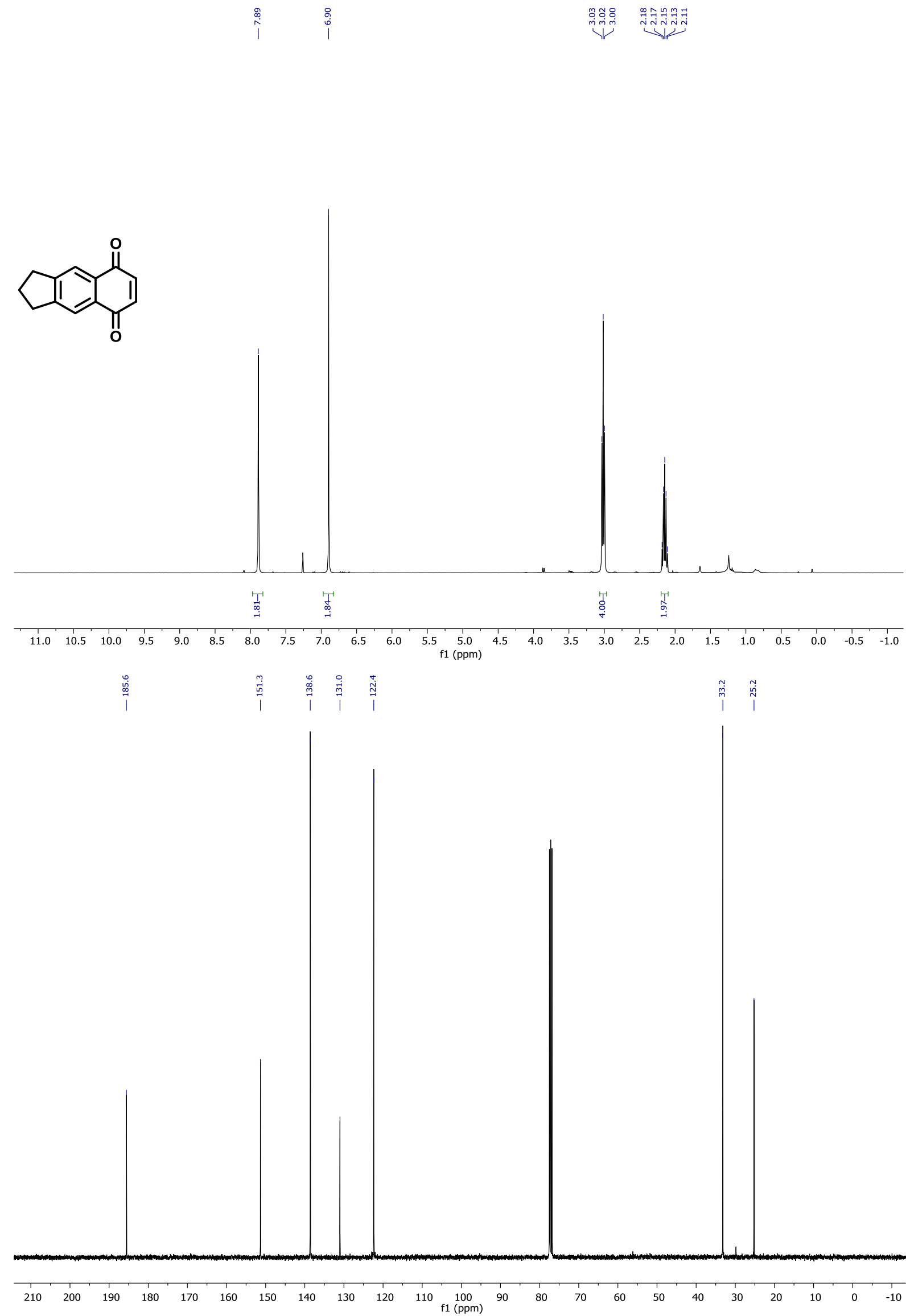


(3ca): 2-Tosyl-2,3-dihydro-1H-benzo[ $f]$ isoindole-5,8-dione

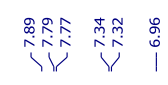

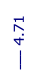

$\stackrel{\substack{\mathfrak{i} \\ \text { I }}}{1}$
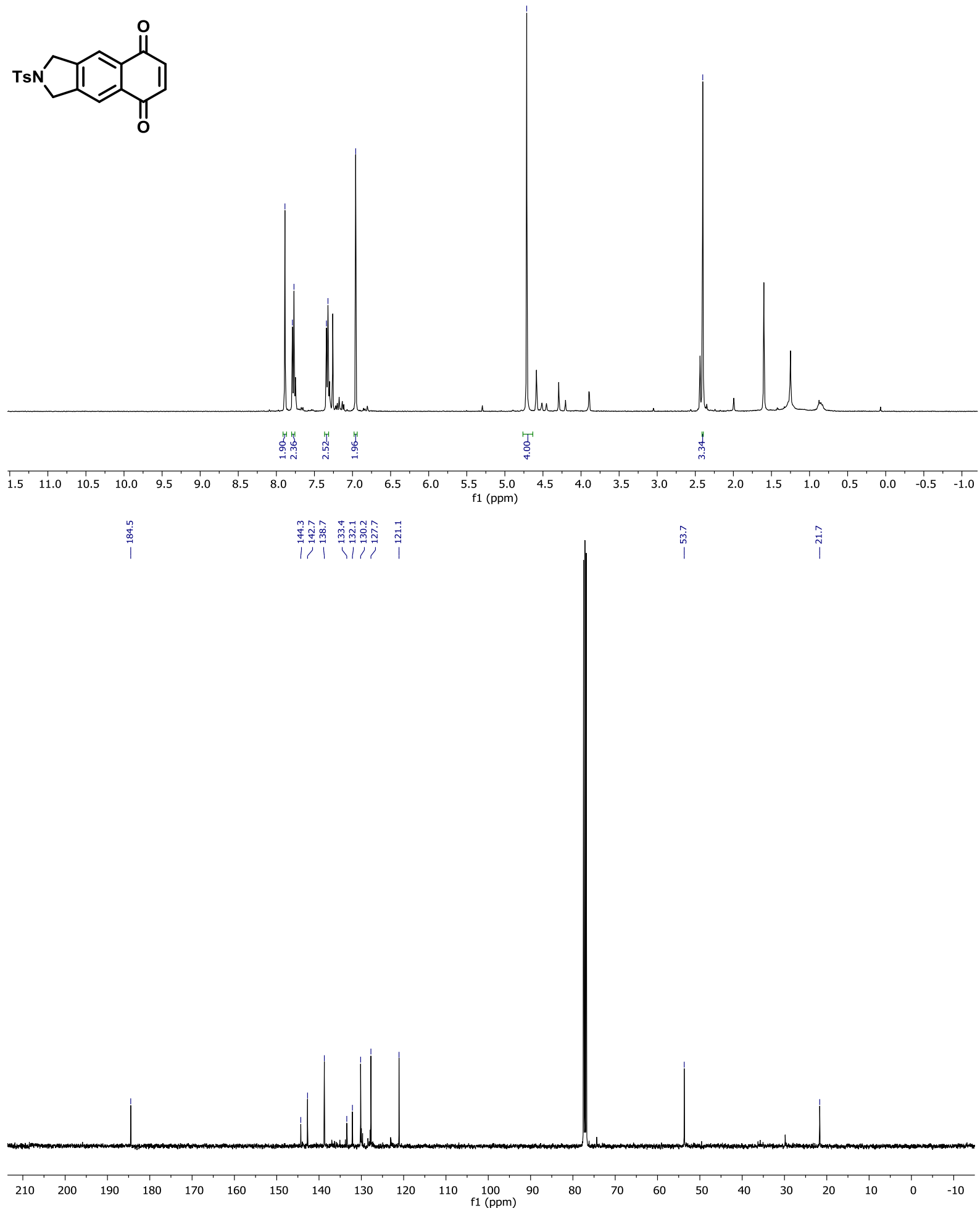

S52 
(3da): 1,3-Dihydronaphtho[2,3-c]furan-5,8-dione
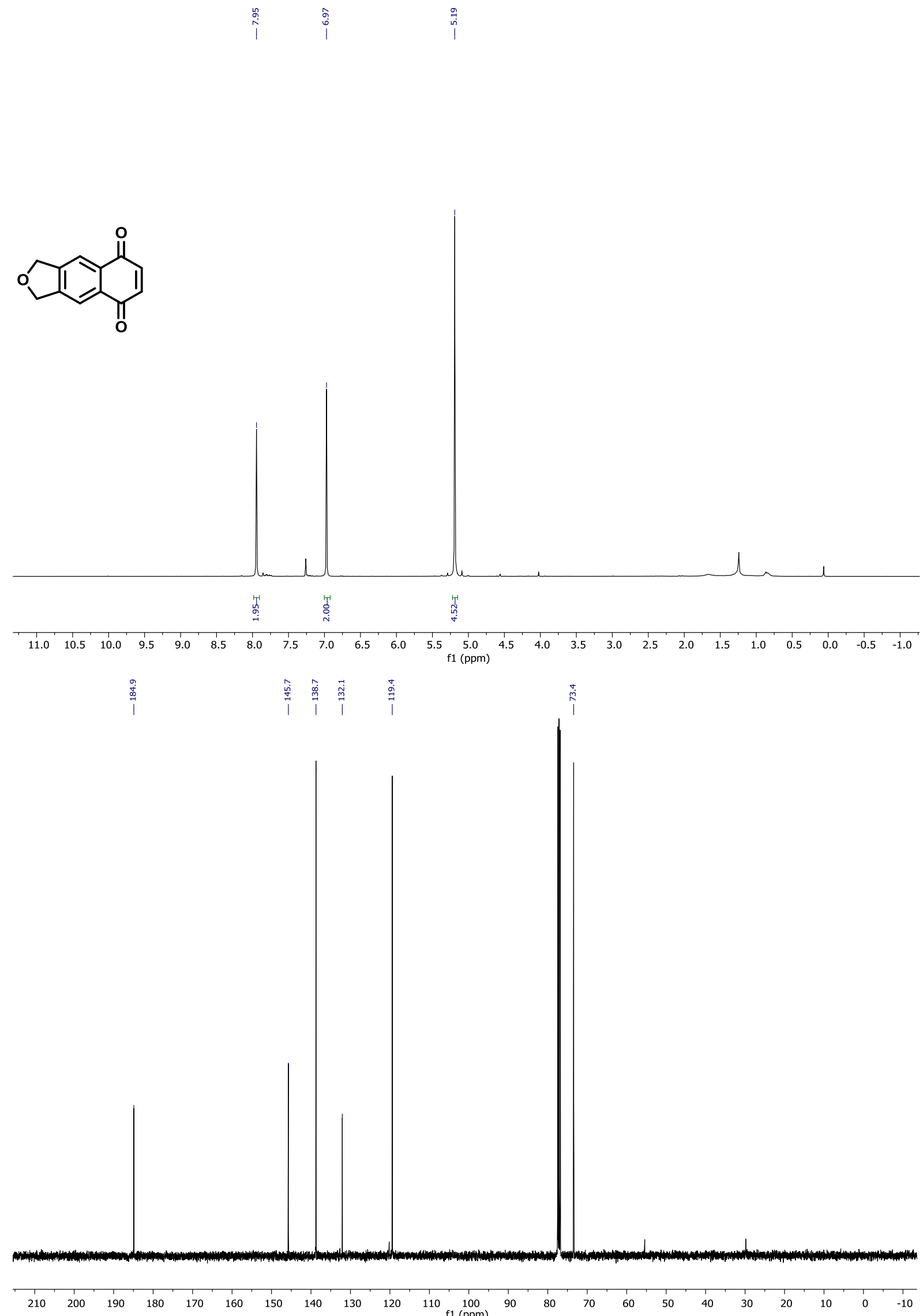
(3ea): Dimethyl 4,9-dimethyl-5,8-dioxo-1,3,5,8-tetrahydro-2H-cyclopenta[b]naphthalene-2,2dicarboxylate

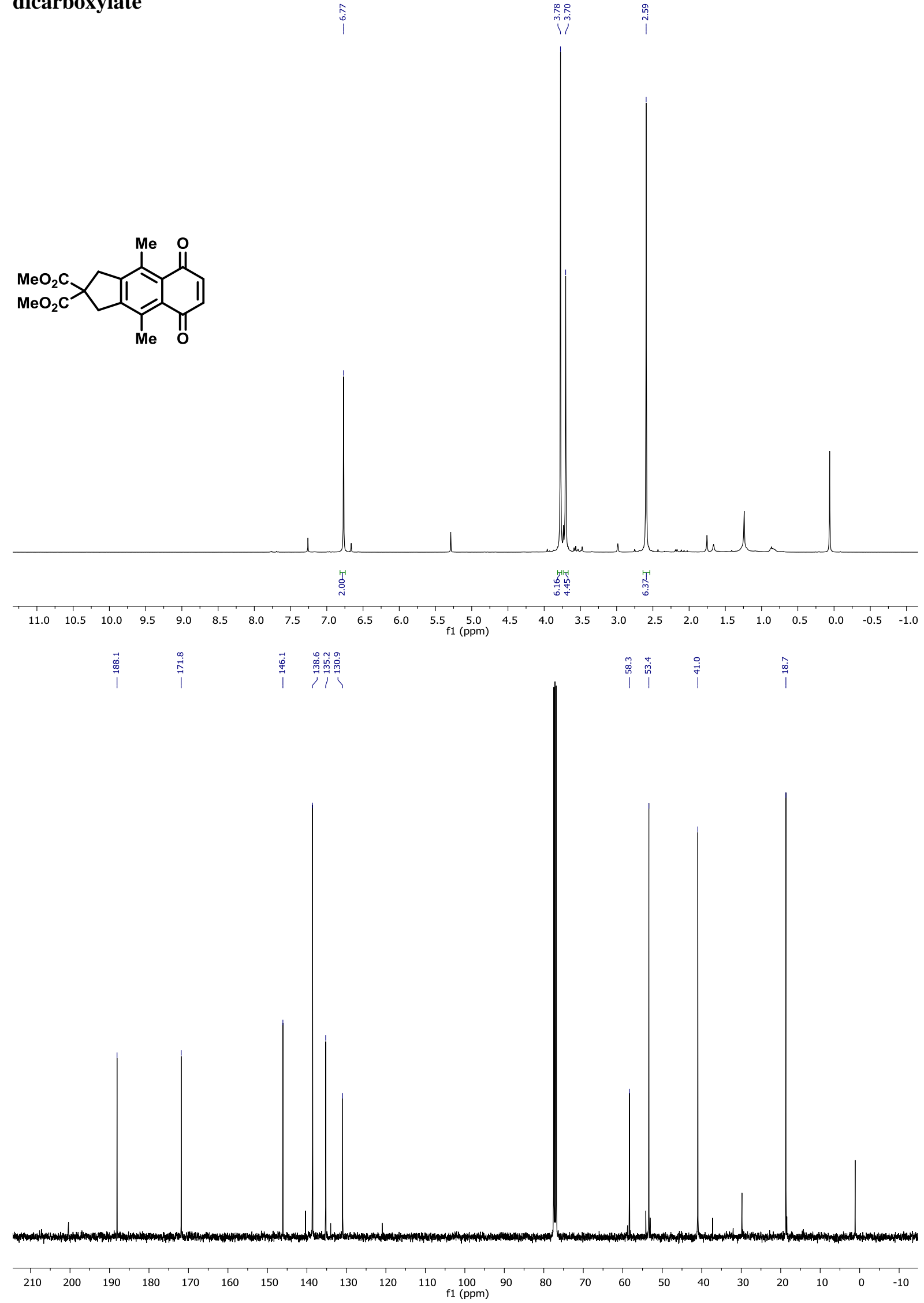


(3fa): 4,9-Dibutyl-2-tosyl-2,3-dihydro-1H-benzo[f]isoindole-5,8-dione
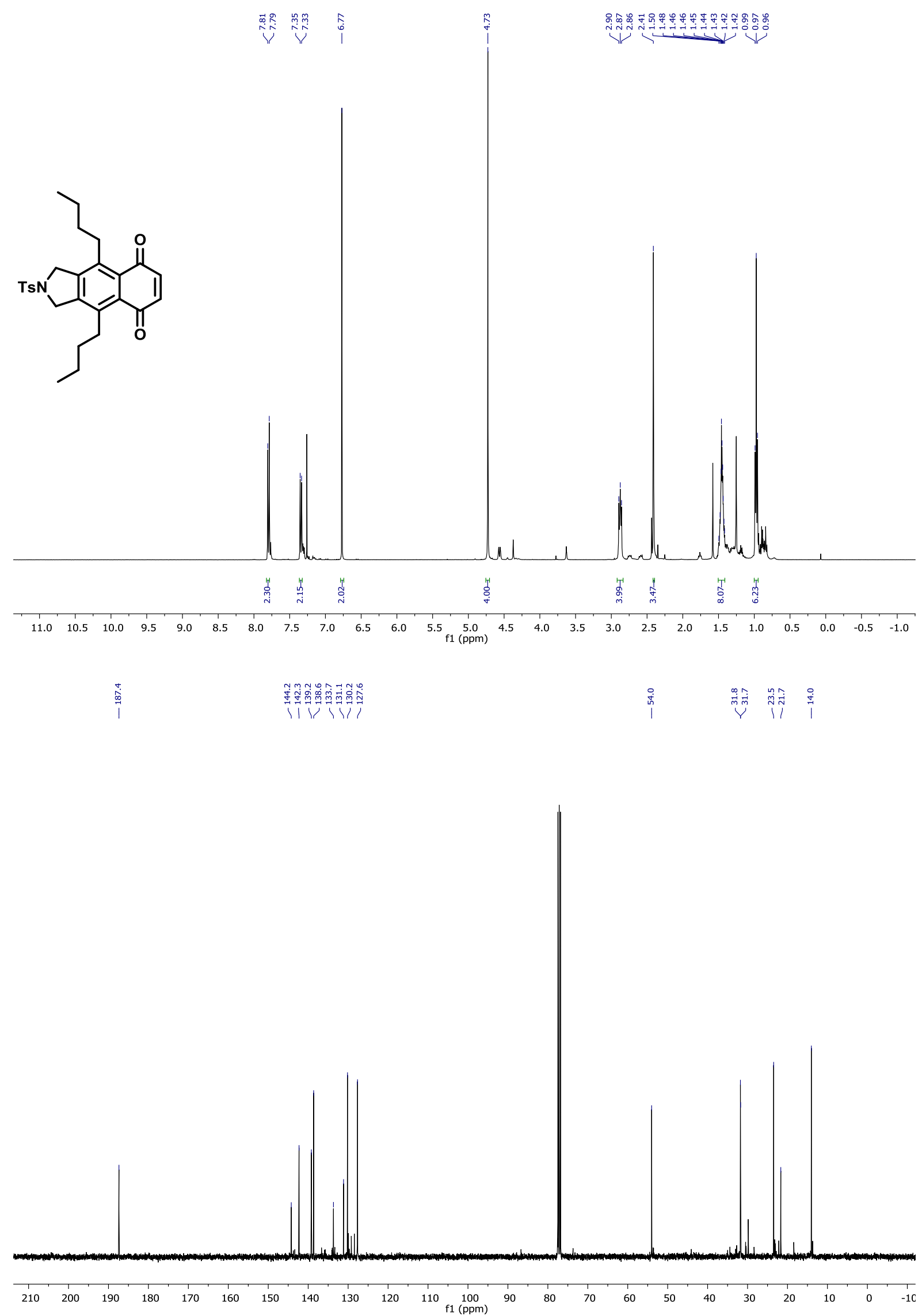
(3ga): Dimethyl 4-methyl-5,8-dioxo-1,3,5,8-tetrahydro-2H-cyclopenta[b]naphthalene-2,2dicarboxylate

$\overbrace{1}^{\text {D. }}$

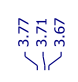

$\stackrel{\leftrightarrow}{\stackrel{\leftrightarrow}{\sim}}$

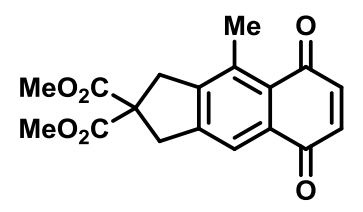

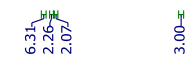
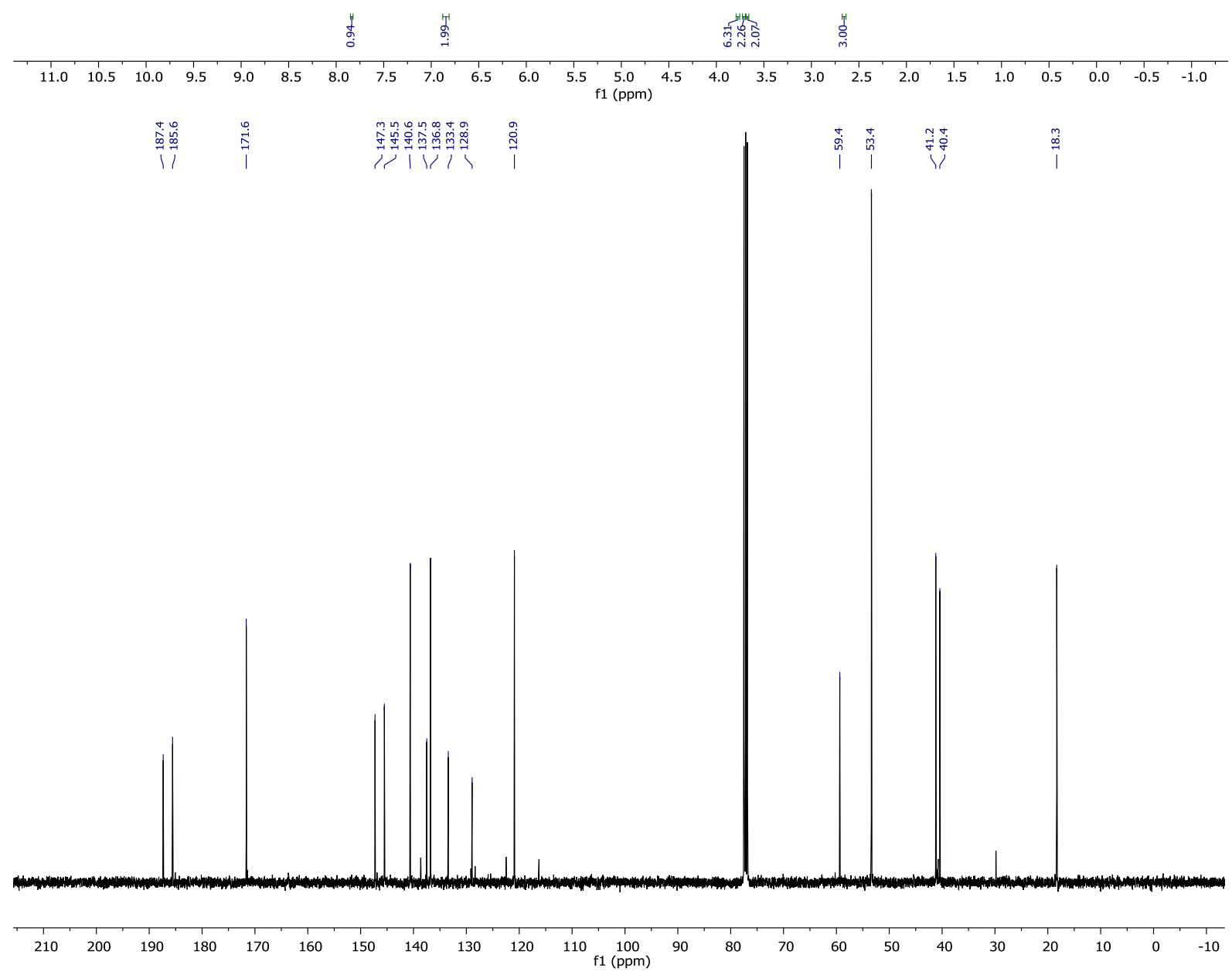

S56 
(3ha): Dimethyl 4-butyl-5,8-dioxo-1,3,5,8-tetrahydro-2H-cyclopenta[b]naphthalene-2,2dicarboxylate

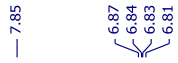<smiles>CCCCCc1c2c(cc3c1C(=O)C=CC3=O)CC(CCCC)(C(C)=O)C2</smiles>
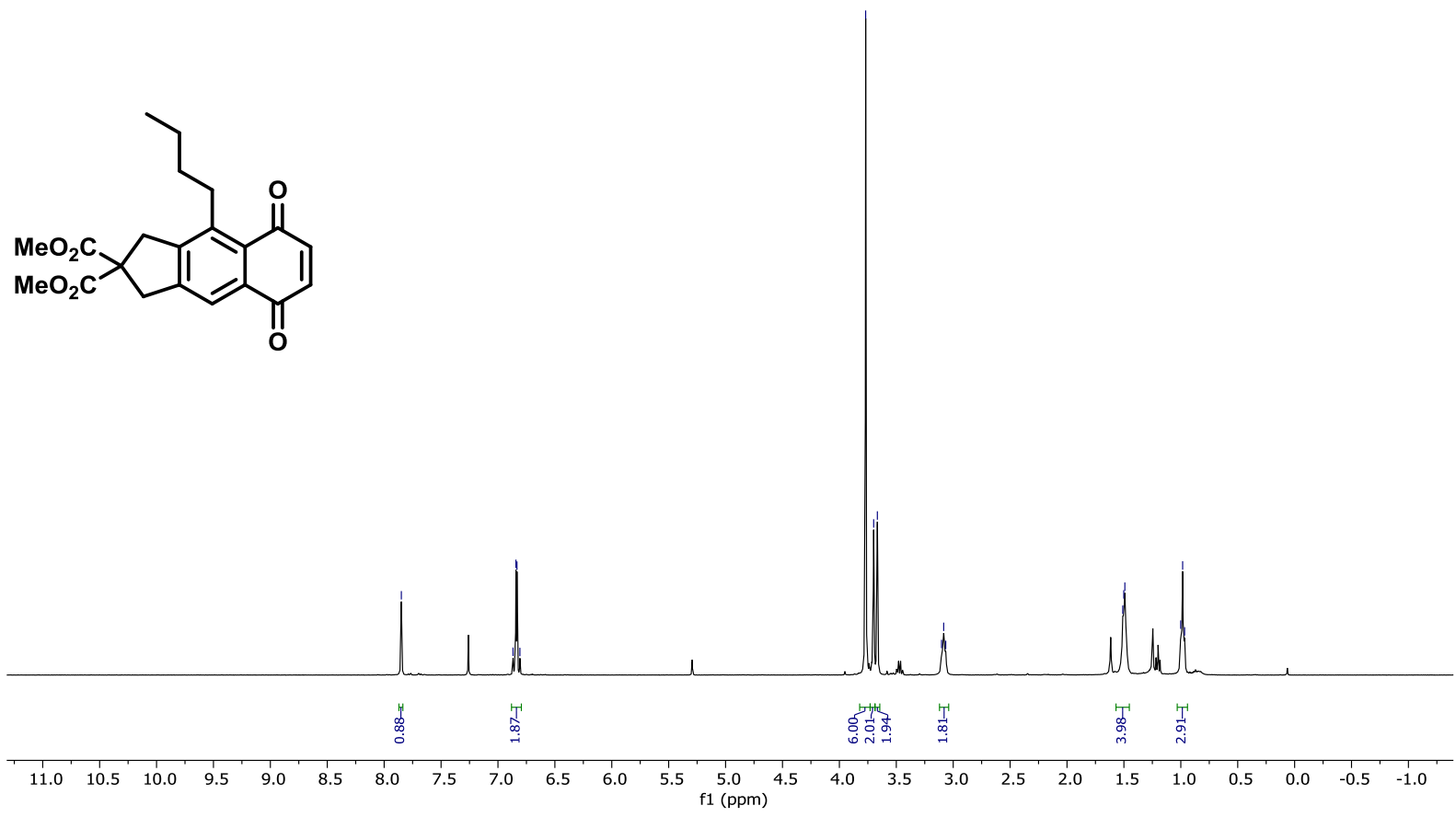
|

|

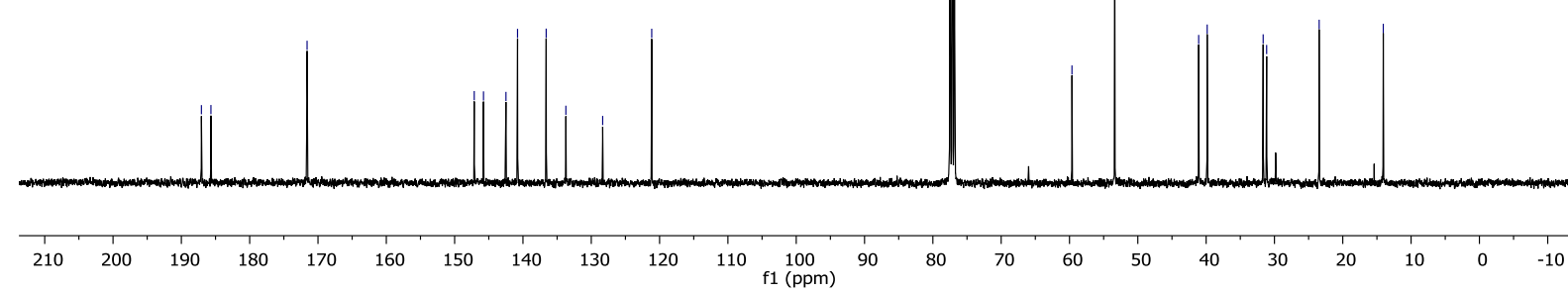


(3ia): Dimethyl 4-((tert-butyldimethylsilyl)oxymethyl)-5,8-dioxo-1,3,5,8-tetrahydro-2Hcyclopenta[$[b]$ naphthalene-2,2-dicarboxylate
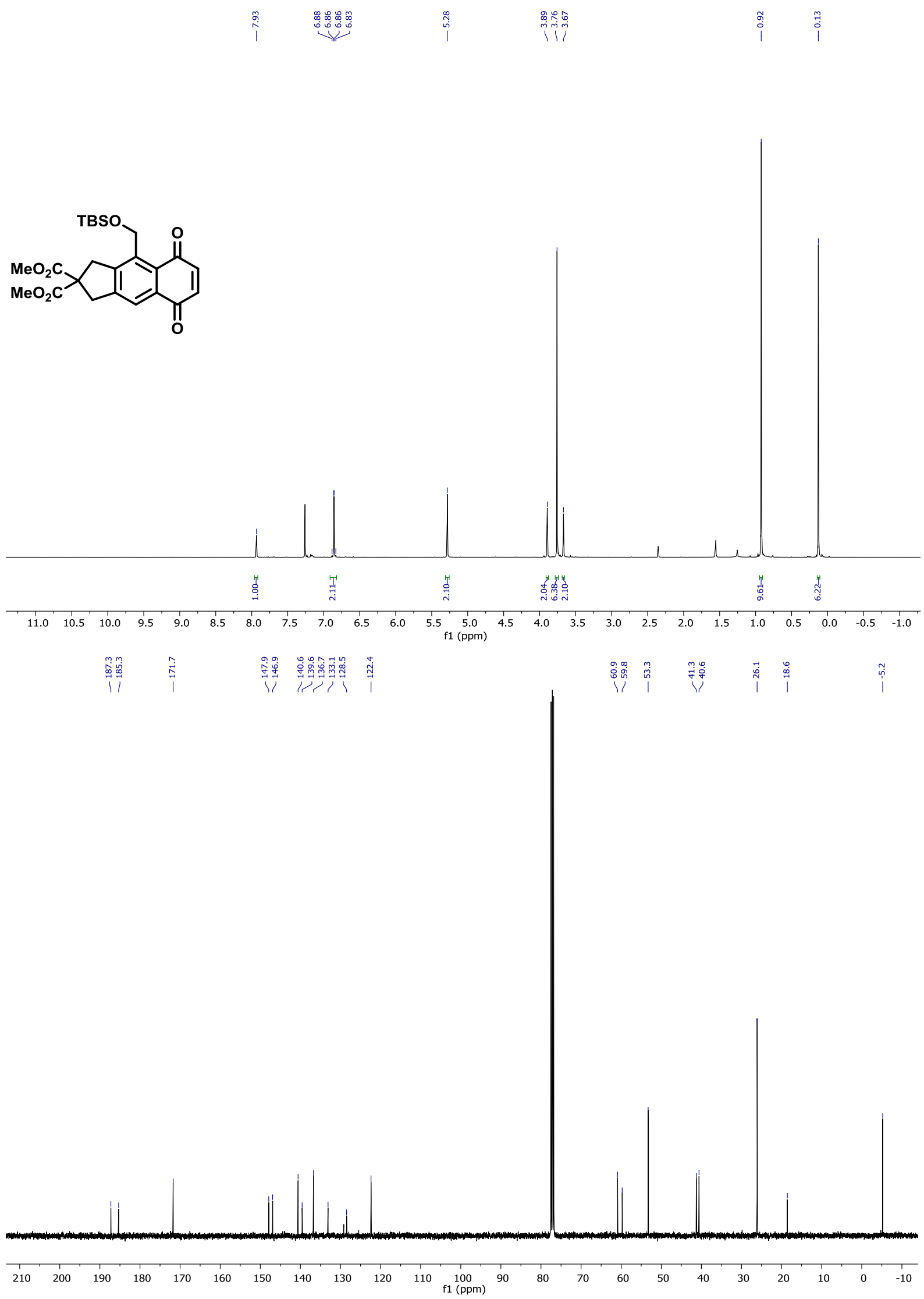
(3ja): Dimethyl 5,8-dioxo-4,9-diphenyl-1,3,5,8-tetrahydro-2H-cyclopenta[b]naphthalene-2,2dicarboxylate

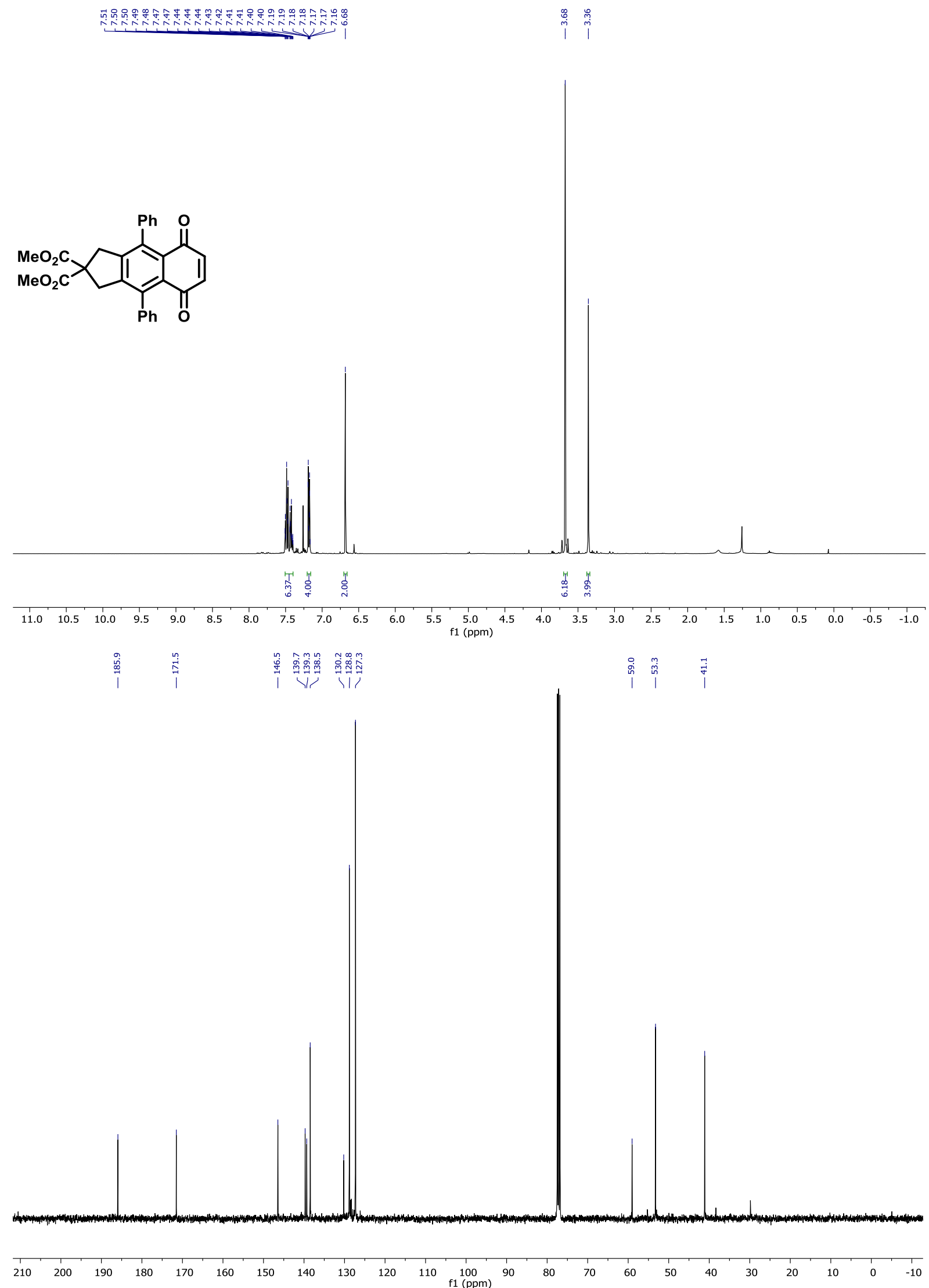


(3ka): Dimethyl 4,9-bis(4-methoxyphenyl)-5,8-dioxo-1,3,5,8-tetrahydro-2H-

cyclopenta $[b]$ naphthalene-2,2-dicarboxylate

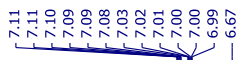
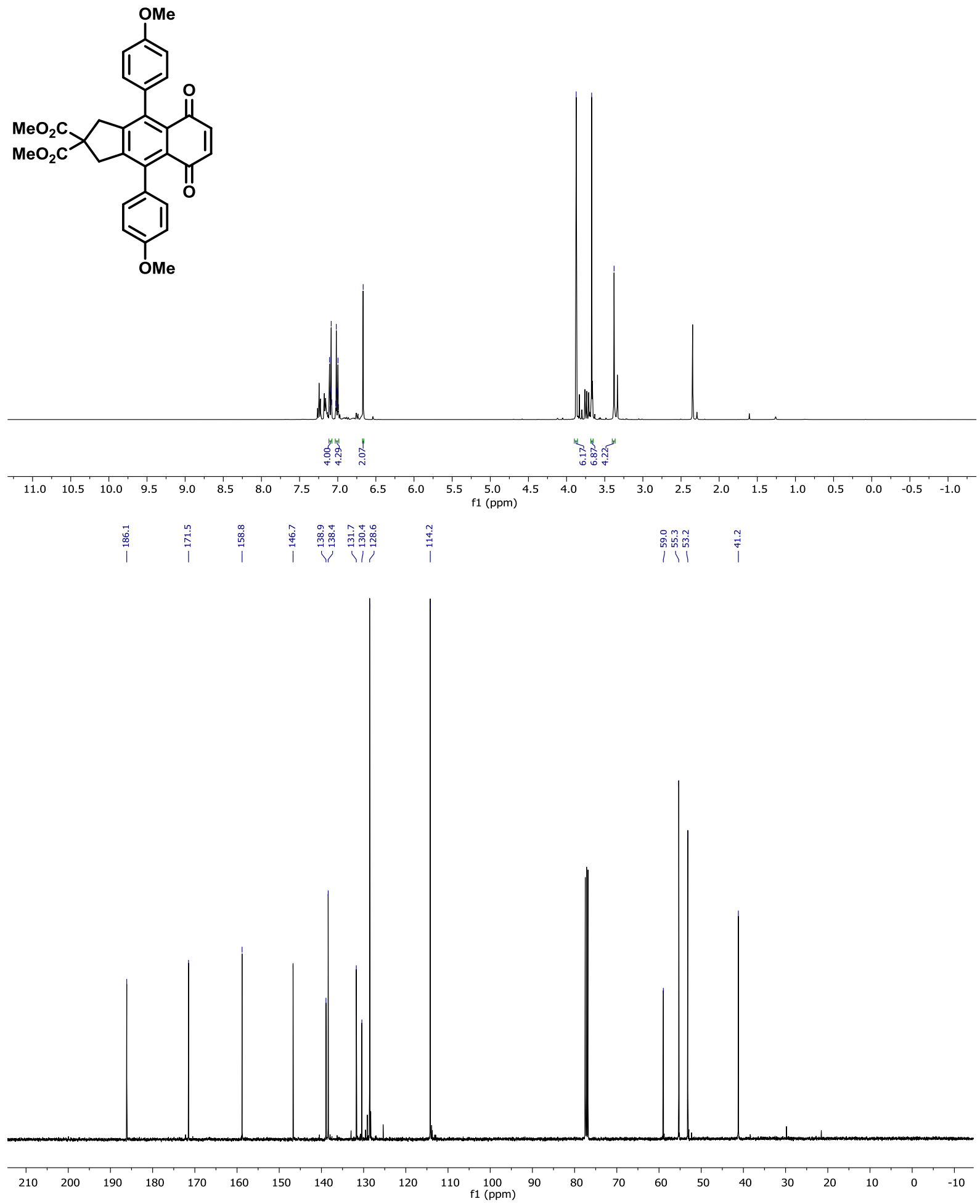
(3la): Dimethyl 4,9-bis(4-fluorophenyl)-5,8-dioxo-1,3,5,8-tetrahydro-2Hcyclopenta[b]naphthalene-2,2-dicarboxylate
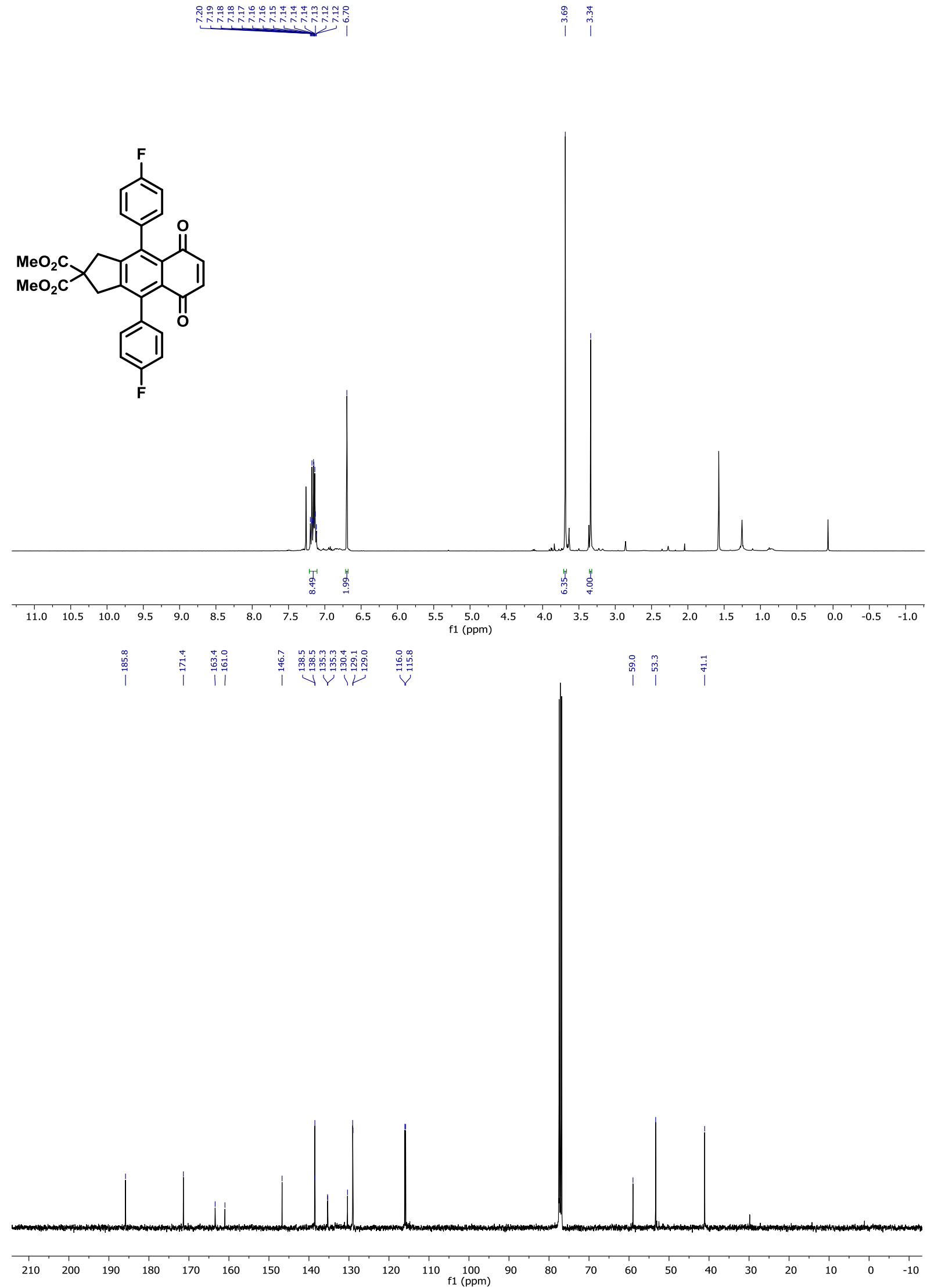
(3ma): 11-Phenyl-5-tosyl-5H-benzo[b]carbazole-7,10-dione

品

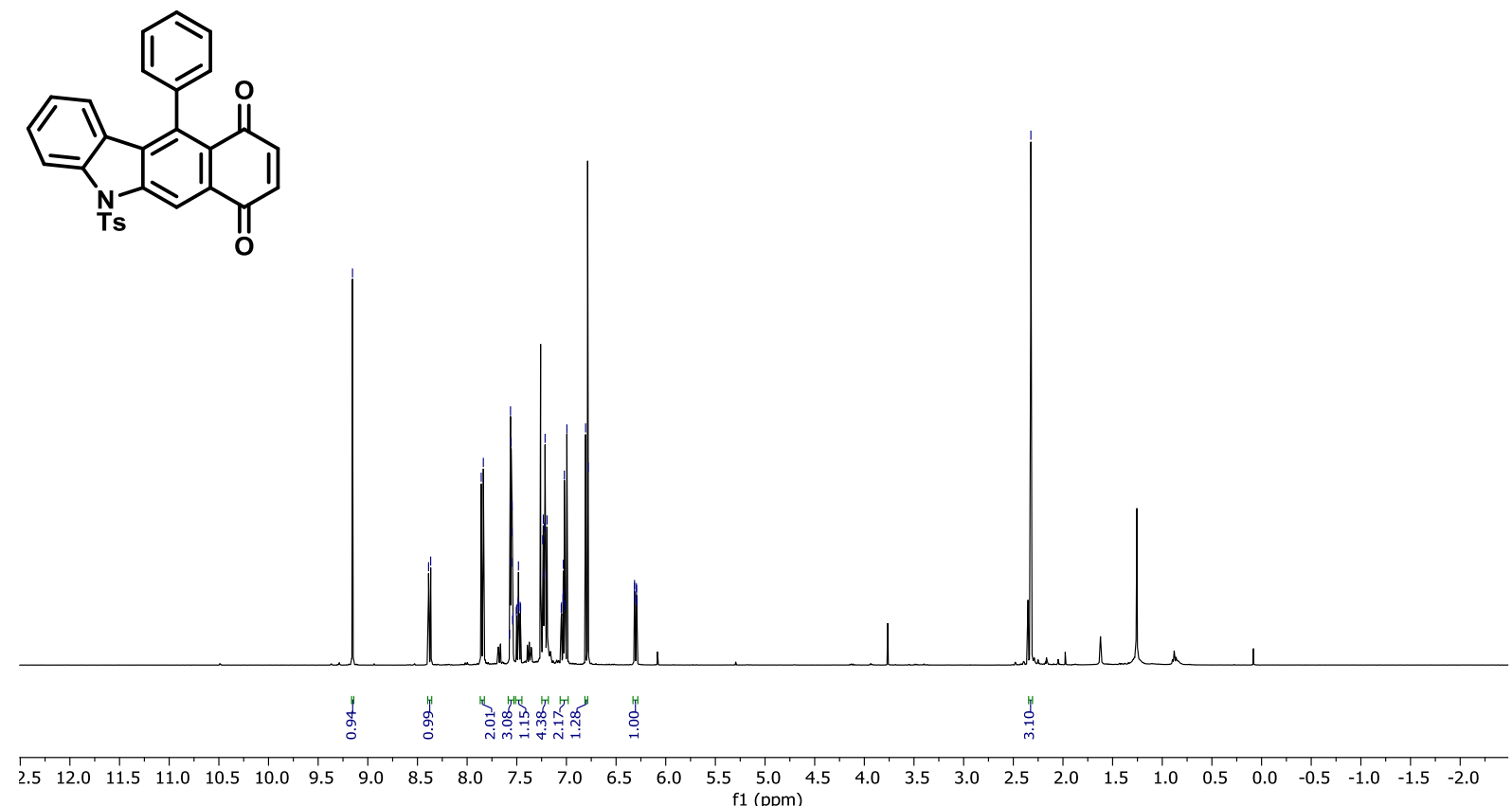

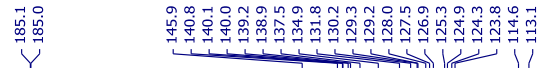

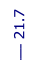

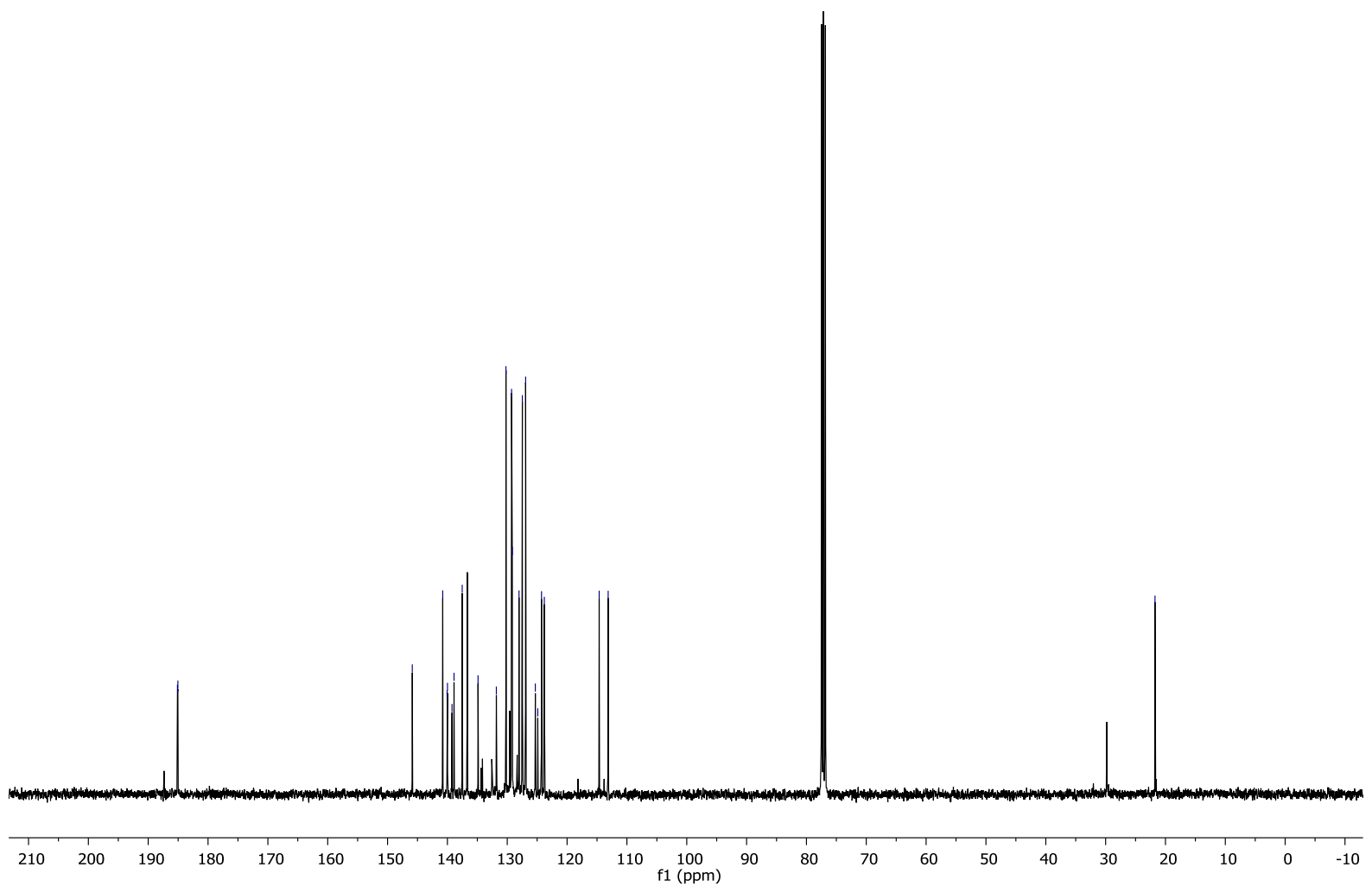


(3na): 11-Phenylnaphtho[2,3-b]benzofuran-7,10-dione

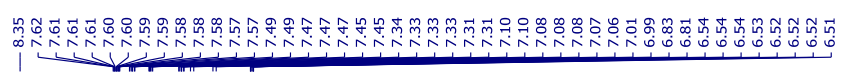
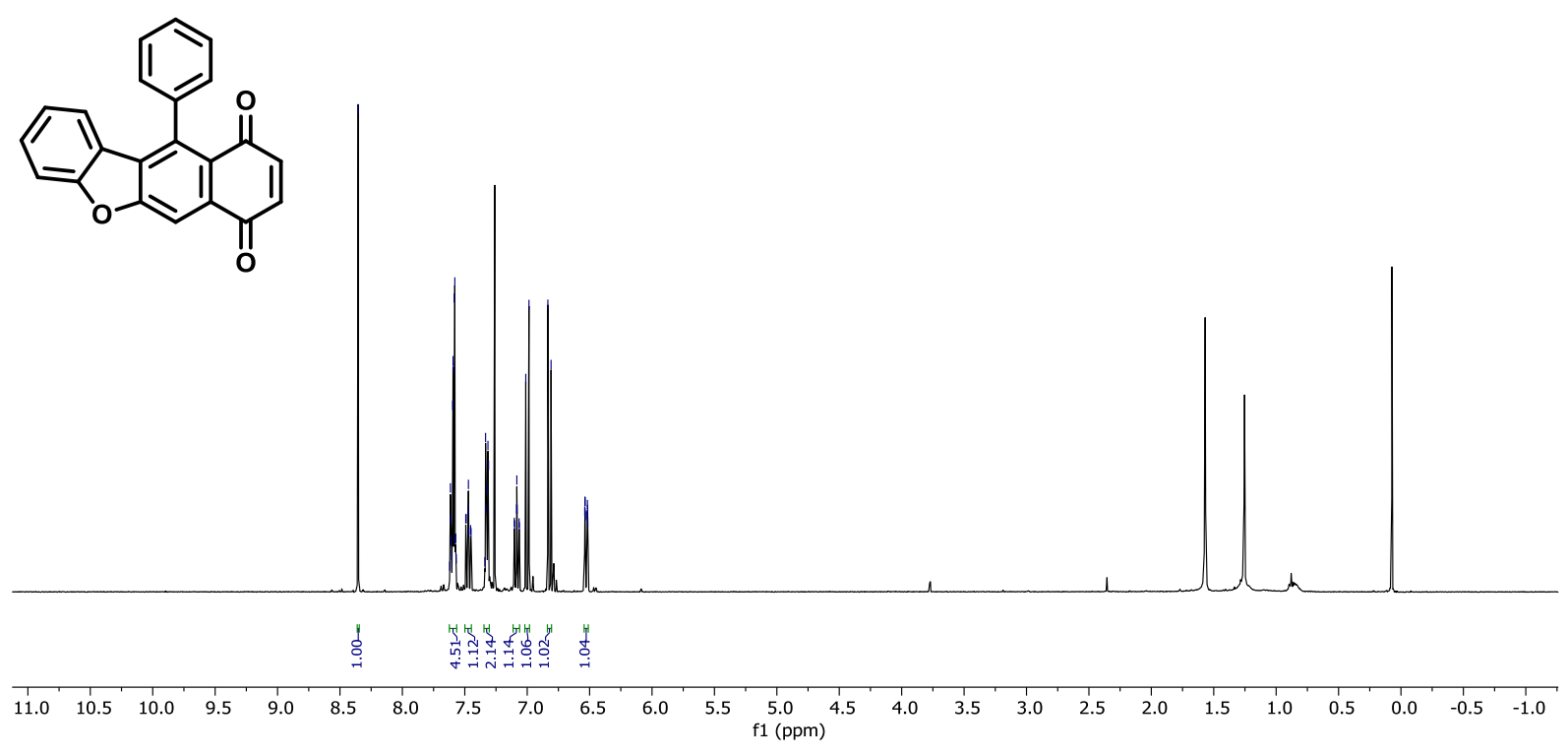

要道

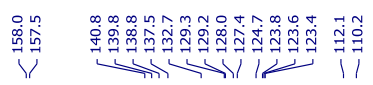

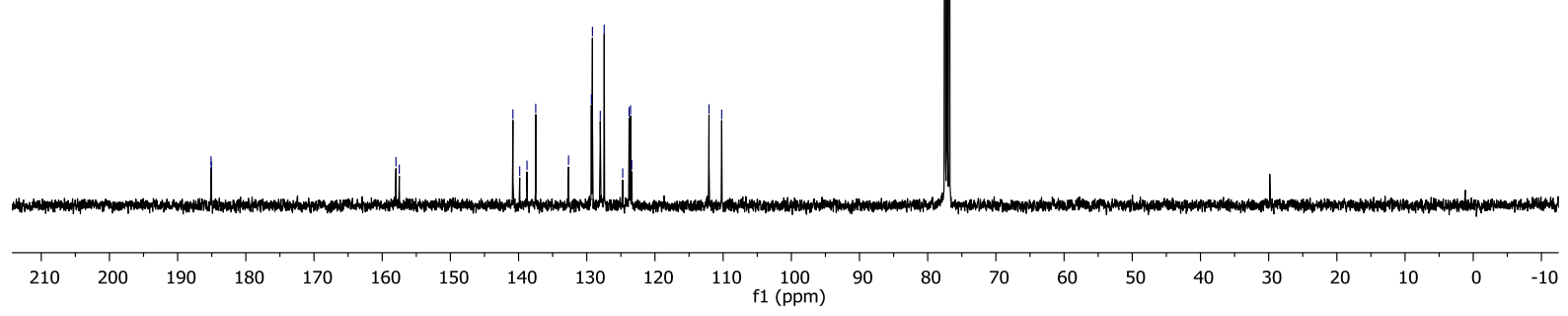

S63 
(3ea'): Dimethyl (4a $\left.R^{*}, 8 \mathrm{a} S^{*}\right)-4,9-d i m e t h y l-5,8-d i o x o-1,3,4 a, 5,8,8 \mathrm{a}-h e x a h y d r o-2 H$ cyclopenta $[b]$ naphthalene-2,2-dicarboxylate*
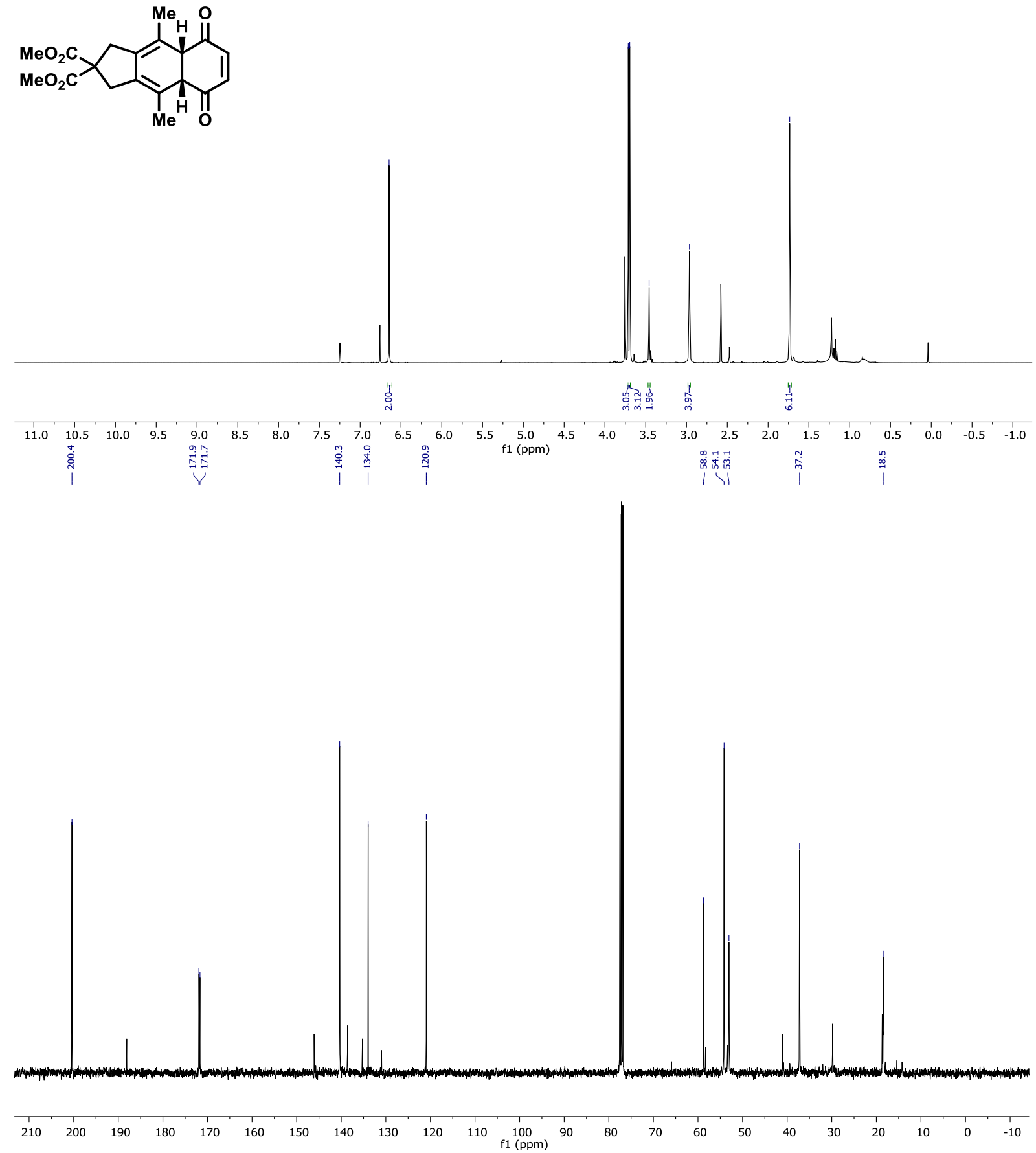

*Contaminated with naphthoquinone 3 ea. 
(5): 2,2-Bis(methoxycarbonyl)-5,8-dioxo-2,3,5,8-tetrahydro- $1 H$-cyclopenta[b]naphthalene-4carboxylic acid

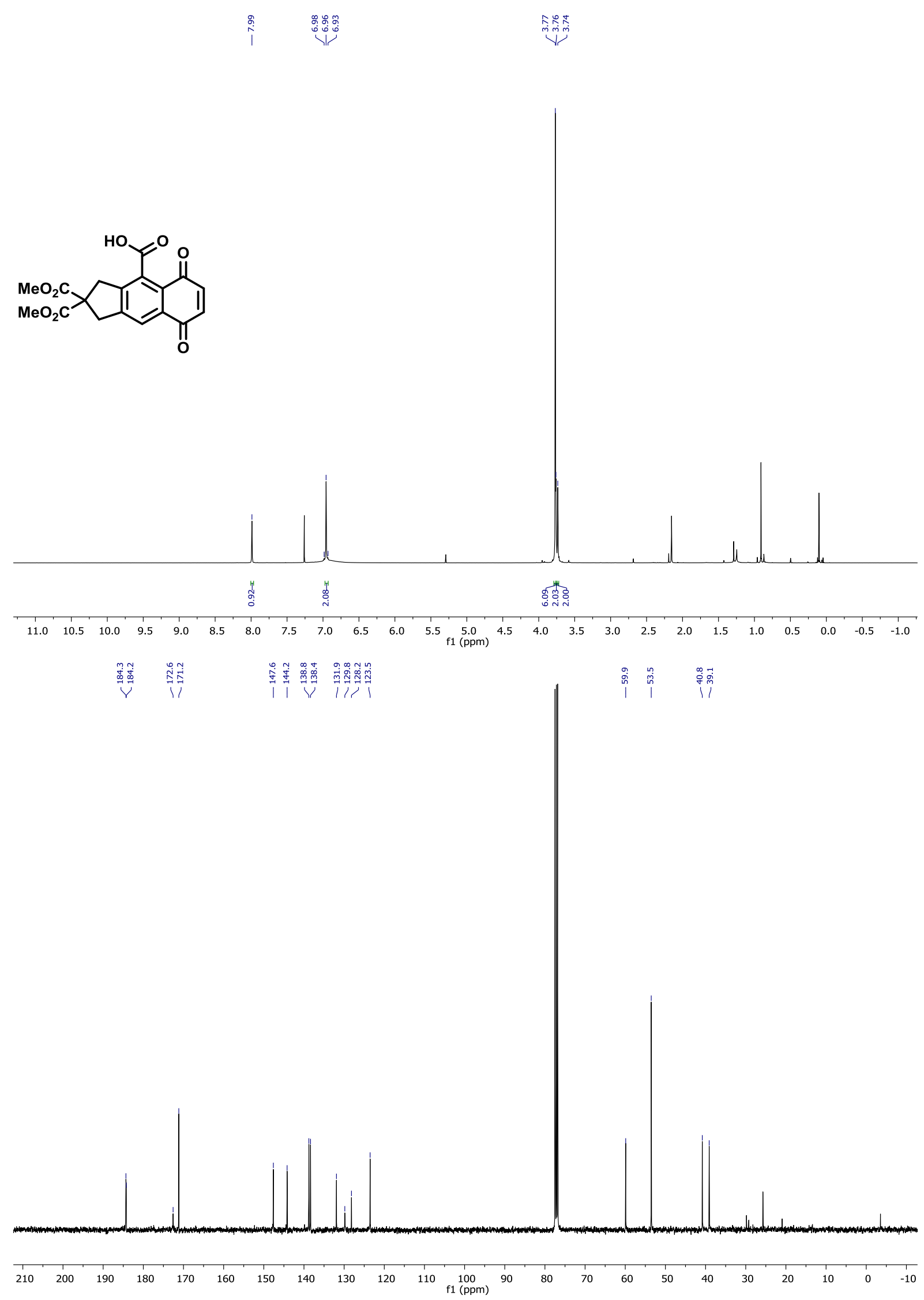


(6): Dimethyl 4-hydroxy-7-methoxy-5,8-dioxo-1,3,5,8-tetrahydro-2H-cyclopenta[b]naphthalene2,2-dicarboxylate<smiles>COC1=CC(=O)c2c(cc3c(c2O)CC(C)(C(C)=O)C3)C1=O</smiles>

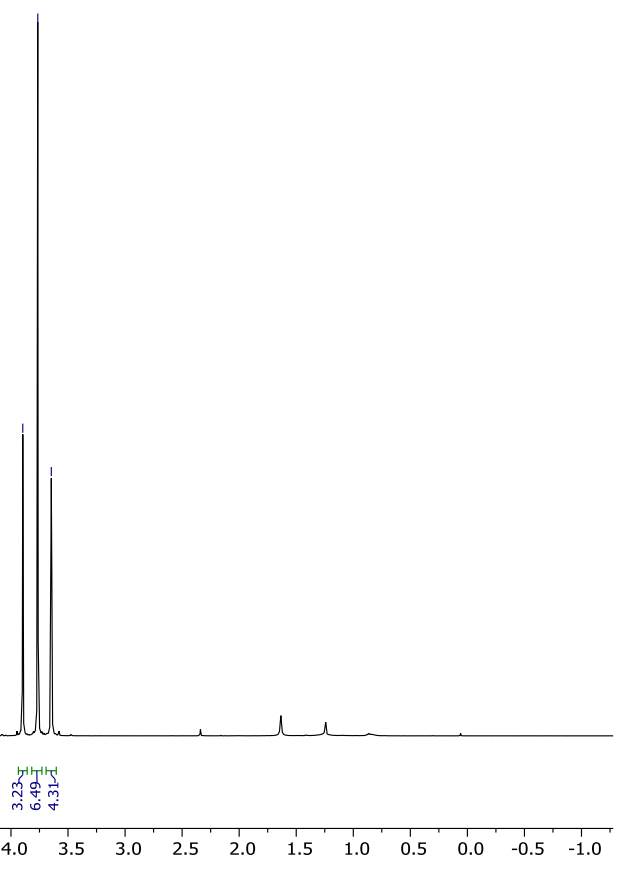

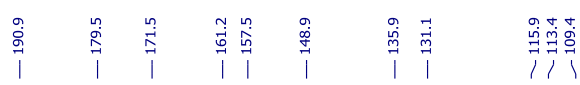
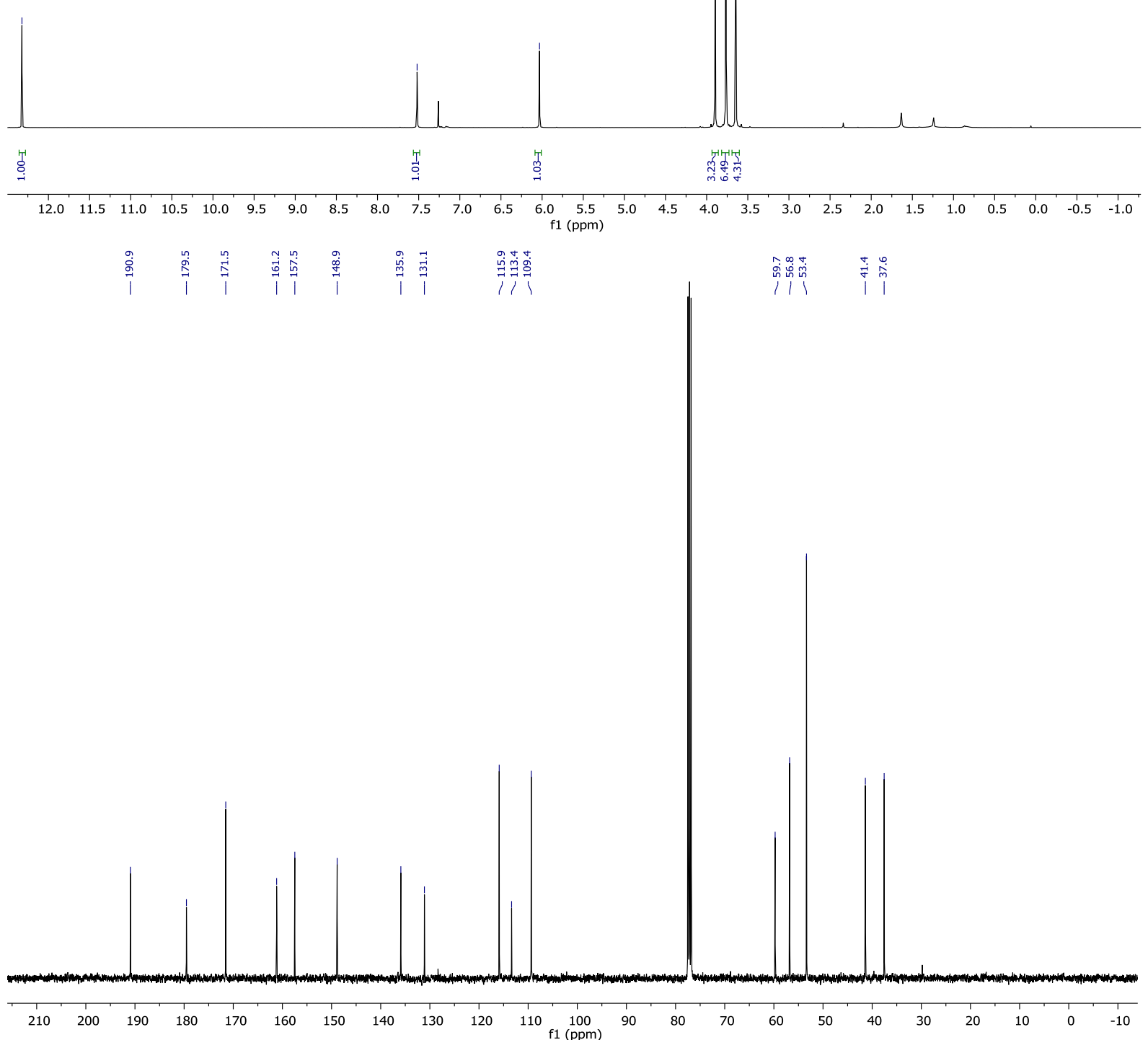
(7): Dimethyl 4,9-dihydroxy-6-methoxy-5,8-dioxo-1,3,5,8-tetrahydro-2Hcyclopenta $[b]$ naphthalene-2,2-dicarboxylate
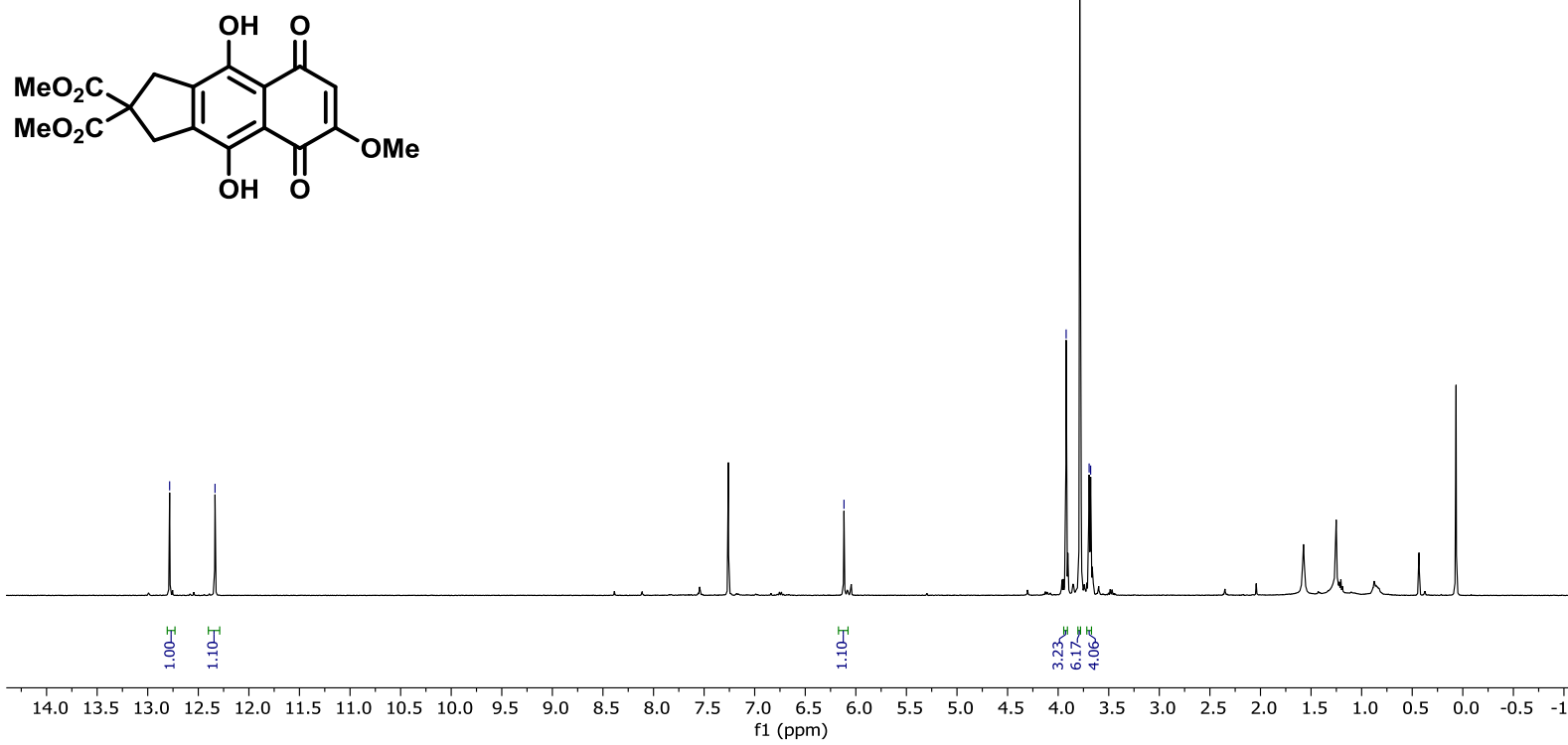

|

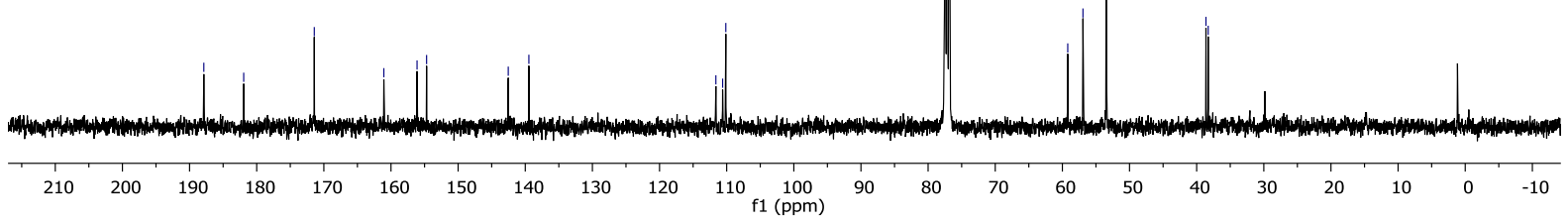


(10): 3-(Benzo[d][1,3]dioxol-5-yl)prop-2-yn-1-ol
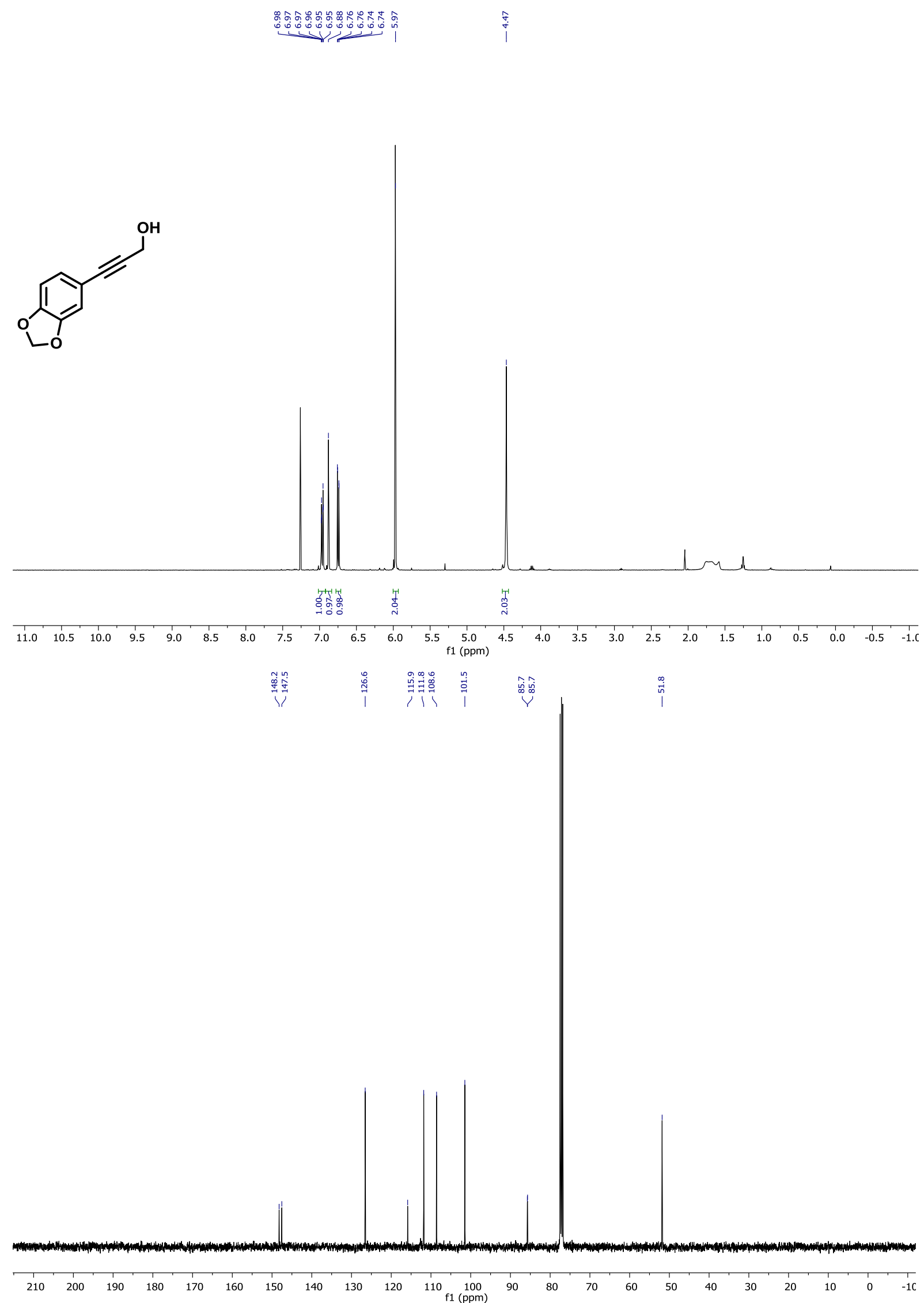
(10): 3-(Benzo[d][1,3]dioxol-5-yl)prop-2-yn-1-yl propiolate

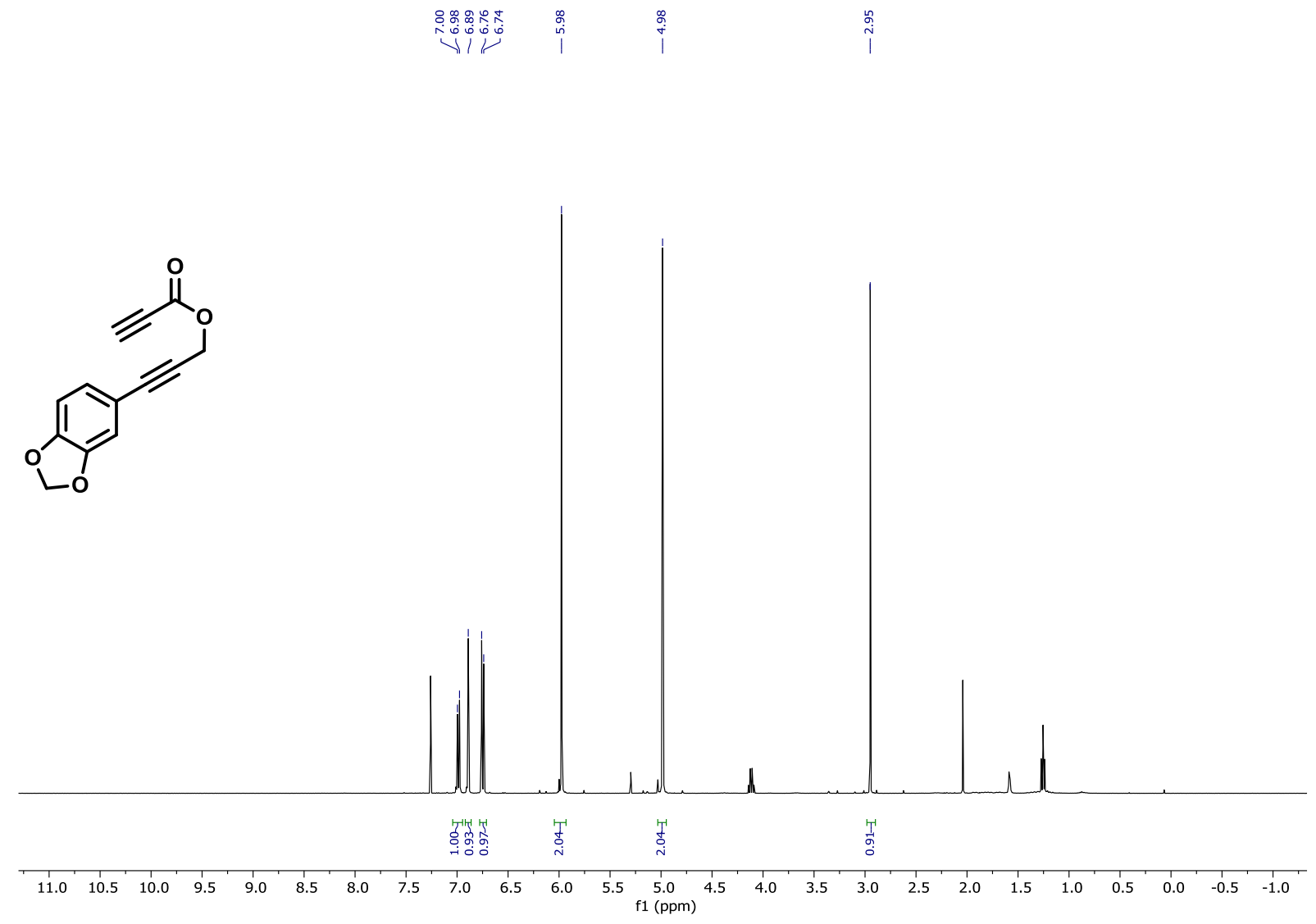

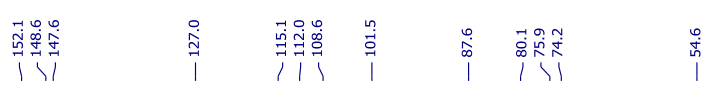

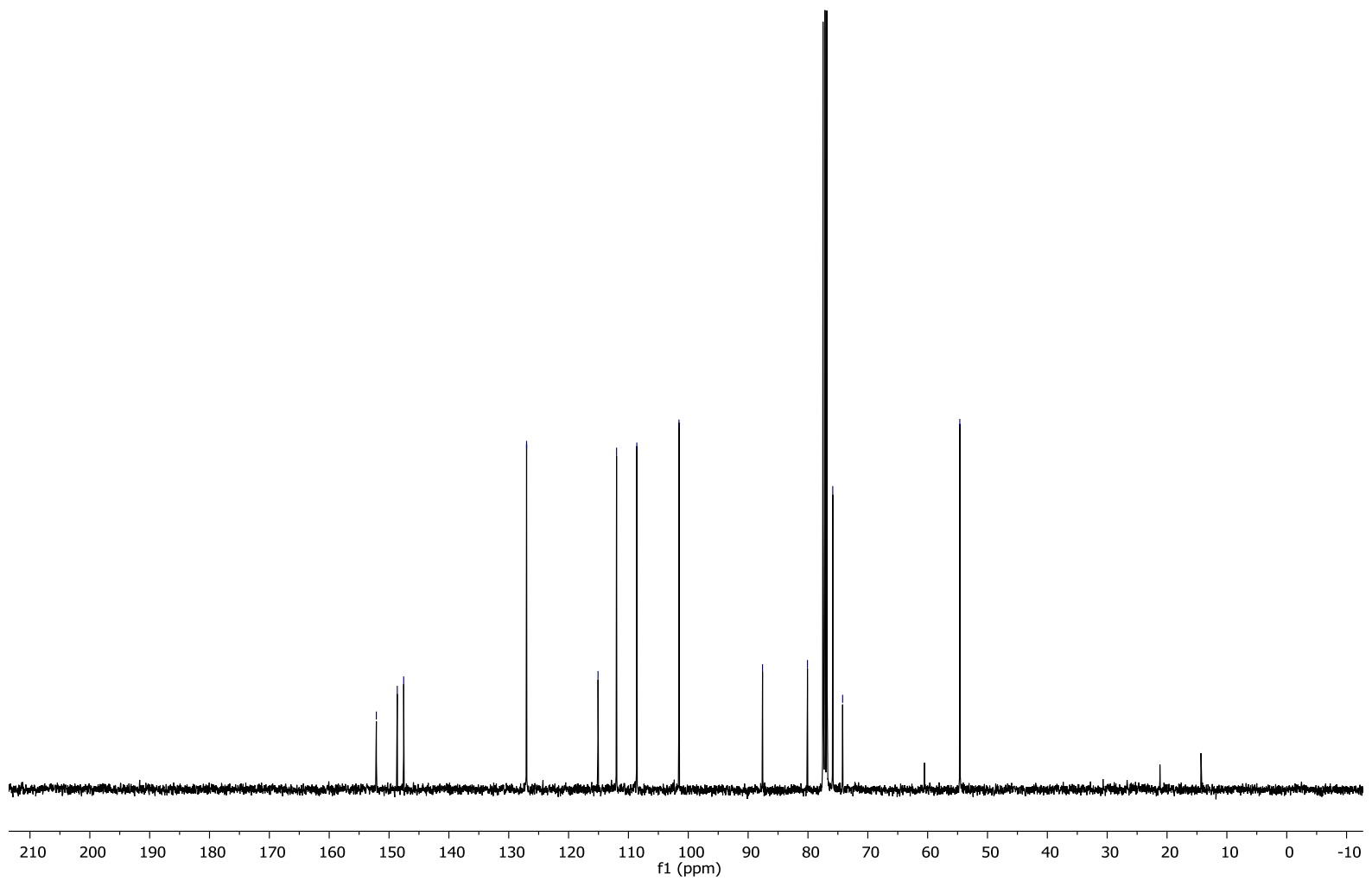




\section{Justicidone}

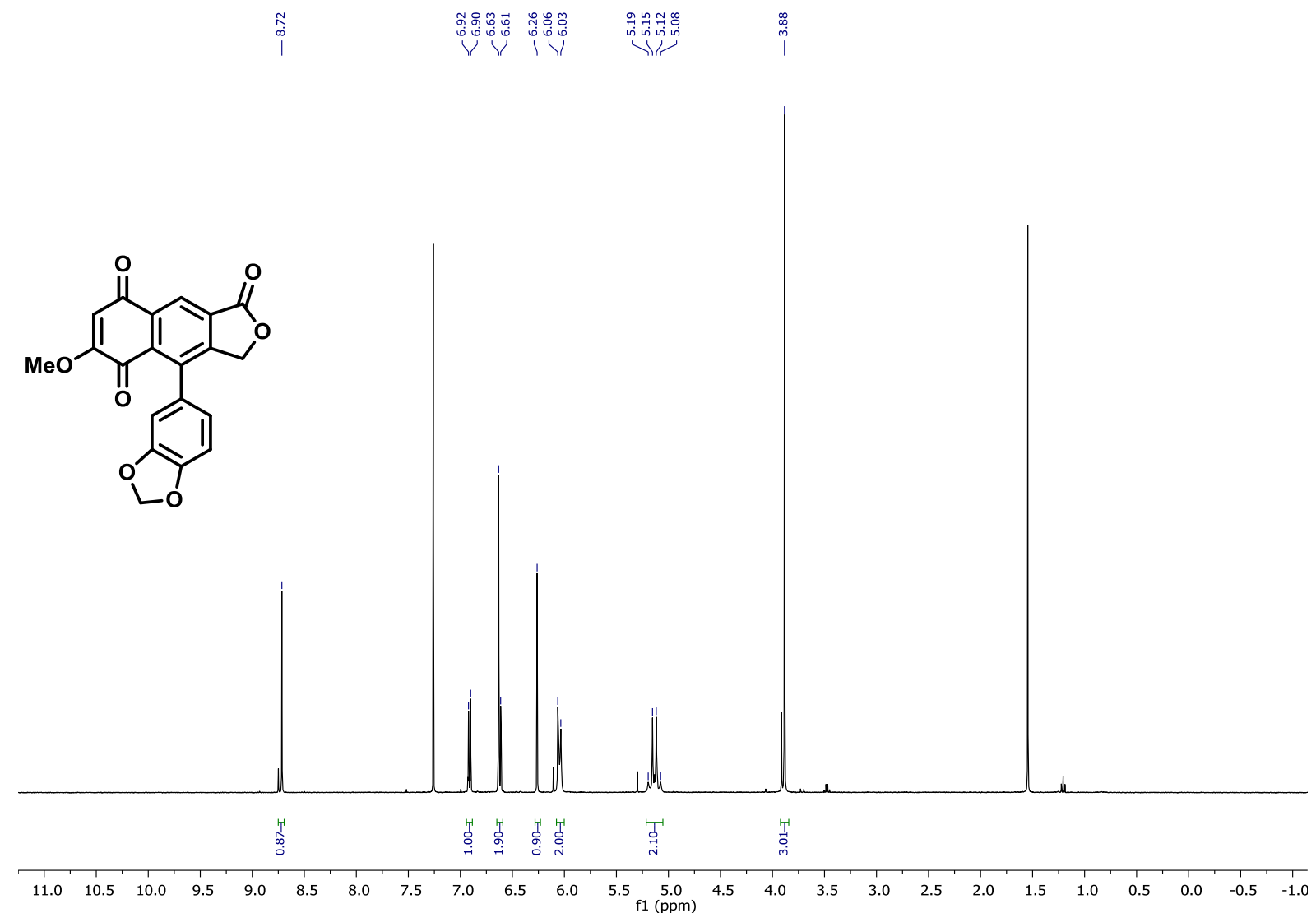

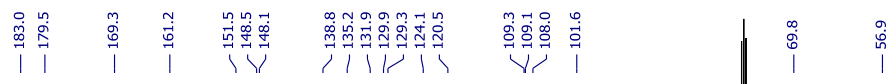

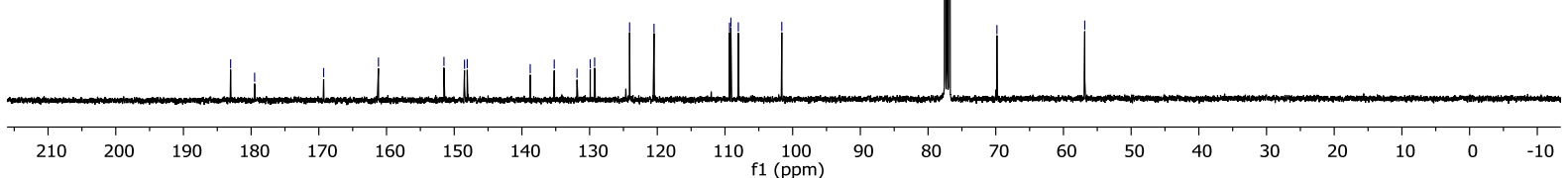




\section{References}

(1) M. H. Shaw, R. A. Croft, W. G. Whittingham, J. F. Bower, J. Am. Chem. Soc. 2015, 137 (25), 8054-8057.

(2) W. N. Chou, M. Pomerantz, J. Org. Chem. 1991, 56 (8), 2762-2769.

(3) J. M. Carney, P. J. Donoghue, W. M. Wuest, O. Wiest, P. Helquist, Org. Lett. 2008, 10 (17), 3903-3906.

(4) J. Lee, K. H. Kim, O. S. Lee, T.-L. Choi, H.-S. Lee, H. Ihee, J.-H. Sohn, J. Org. Chem. 2016, 81 (17), 7591-7596.

(5) A.-F. Tran-Van, E. Huxol, J. M. Basler, M. Neuburger, J.-J. Adjizian, C. P. Ewels, H. A. Wegner, Org. Lett. 2014, 16 (6), 1594-1597.

(6) M. Wilking, C. Mück-Lichtenfeld, C. G. Daniliuc, U. Hennecke, J. Am. Chem. Soc. 2013, 135 (22), 8133-8136.

(7) V. Richard, M. Ipouck, D. S. Mérel, S. Gaillard, R. J. Whitby, B. Witulski, J.-L. Renaud, Chem. Commun. 2013, 50 (5), 593-595.

(8) S. Levin, R. R. Nani, S. E. Reisman, Org. Lett. 2010, 12 (4), 780-783.

(9) K. Yamashita, Y. Nagashima, Y. Yamamoto, H. Nishiyama, Chem. Commun. 2011, 47 (41), $11552-11554$.

(10) G. S. Sinclair, T. Yang, S. Wang, W. H. Chen, D. J. Schipper, Org. Lett. 2017, 19 (4), 802-805.

(11) Y. Komine, A. Kamisawa, K. Tanaka, Org. Lett. 2009, 11 (11), 2361-2364.

(12) V. Sridharan, L. Fan, S. Takizawa, T. Suzuki, H. Sasai, Org. Biomol. Chem. 2013, 11 (35), 59365943.

(13) S. Kim, R. Matsubara, M. Hayashi, J. Org. Chem. 2019, 84 (5), 2997-3003.

(14) M. H. Ali, M. Niedbalski, G. Bohnert, D. Bryant, Synth. Commun. 2006, 36 (12), 1751-1759.

(15) Y. Fujiwara, V. Domingo, I. B. Seiple, R. Gianatassio, M. Del Bel, P. S. Baran, J. Am. Chem. Soc. 2011, 133 (10), 3292-3295.

(16) M. A. Brimble, M. T. Brimble, J. J. Gibson, J. Chem. Soc. Perkin 1 1989, No. 1, 179-184.

(17) B. Ge, D. Wang, W. Dong, P. Ma, Y. Li, Y. Ding, Tetrahedron Lett. 2014, 55 (40), 5443-5446.

(18) M. Nakazaki, K. Naemura, J. Org. Chem. 1981, 46 (1), 106-111.

(19) H. Miyamura, M. Shiramizu, R. Matsubara, S. Kobayashi, Angew. Chem. Int. Ed. 2008, 47 (42), 8093-8095.

(20) H. Riering, H. J. Schäfer, Chem. Ber. 1994, 127 (5), 859-873.

(21) E. Schleusener, C. H. Eugster, Helv. Chim. Acta 1972, 55 (3), 986-1002.

(22) A. S. Kalgutkar, K. R. Kozak, B. C. Crews, G. P. Hochgesang, L. J. Marnett, J. Med. Chem. 1998, 41 (24), 4800-4818.

(23) S. Kezuka, S. Tanaka, T. Ohe, Y. Nakaya, R. Takeuchi, J. Org. Chem. 2006, 71 (2), 543-552.

(24) Y. Yin, W. Ma, Z. Chai, G. Zhao, G. J. Org. Chem. 2007, 72 (15), 5731-5736. 
(25) B. Witulski, C. Alayrac, C. Angew. Chem. Int. Ed. 2002, 41 (17), 3281-3284.

(26) J. Chen, J. W. Kampf, A. J. McNeil, Langmuir 2010, 26 (16), 13076-13080.

(27) L. R. Smith, N. Mahoney, R. J. Molyneux, J. Nat. Prod. 2003, 66 (2), 169-176.

(28) M. Lamblin, G. Naturale, J. Dessolin, F.-X. Felpin, Synlett 2012, 23 (11), 1621-1624.

(29) S. Berhe, A. Slupe, C. Luster, H. A. Charlier, D. L. Warner, L. H. Zalkow, E. M. Burgess, N. M. Enwerem, O. Bakare, Bioorg. Med. Chem. 2010, 18 (1), 134-141.

(30) J. A. Varela, S. G. Rubín, C. González-Rodríguez, L. Castedo, C. Saá, C. J. Am. Chem. Soc. 2006, $128(29), 9262-9263$.

(31) J. A. Pérez, C. Boluda, H. López, J. M. Trujillo, Chem. Pharm. Bull. (Tokyo) 2004, 52 (1), 130131. 